\title{
Oxidative Stress and Non-Alcoholic Fatty Liver Disease: Effects of Omega-3 Fatty Acid Supplementation
}

\author{
Jinchunzi Yang ${ }^{1, \dagger}{ }^{\dagger}$ Marta Fernández-Galilea ${ }^{1,2,3, \dagger}{ }^{+}$Leyre Martínez-Fernández ${ }^{1}$, \\ Pedro González-Muniesa ${ }^{1,2,3,4}$ (D), Adriana Pérez-Chávez ${ }^{1}$, J. Alfredo Martínez ${ }^{1,2,3,4}$ (iD) and \\ Maria J. Moreno-Aliaga 1,2,3,4,*(D) \\ 1 Centre for Nutrition Research, School of Pharmacy and Nutrition, University of Navarra, 31008 Pamplona, \\ Spain; jyang@alumni.unav.es (J.Y.); mfgalilea@unav.es (M.F.-G.); lmartinez.7@alumni.unav.es (L.M.-F.); \\ pgonmun@unav.es (P.G.-M.); aperez.39@alumni.unav.es (A.P.-C.); jalfmtz@unav.es (J.A.M.) \\ 2 Department of Nutrition, Food Science and Physiology, School of Pharmacy and Nutrition, \\ University of Navarra, 31008 Pamplona, Spain \\ 3 IDISNA, Navarra's Health Research Institute, 31008 Pamplona, Spain \\ 4 CIBERobn Physiopathology of Obesity and Nutrition, Centre of Biomedical Research Network, ISCIII, \\ 28029 Madrid, Spain \\ * Correspondence: mjmoreno@unav.es; Tel.: +34-948-425-600 \\ + Equal authorship.
}

Received: 29 March 2019; Accepted: 15 April 2019; Published: 18 April 2019

\begin{abstract}
Aging is a complex phenomenon characterized by the progressive loss of tissue and organ function. The oxidative-stress theory of aging postulates that age-associated functional losses are due to the accumulation of ROS-induced damage. Liver function impairment and non-alcoholic fatty liver disease (NAFLD) are common among the elderly. NAFLD can progress to non-alcoholic steatohepatitis (NASH) and evolve to hepatic cirrhosis or hepatic carcinoma. Oxidative stress, lipotoxicity, and inflammation play a key role in the progression of NAFLD. A growing body of evidence supports the therapeutic potential of omega-3 polyunsaturated fatty acids (n-3 PUFA), mainly docosahaexenoic (DHA) and eicosapentaenoic acid (EPA), on metabolic diseases based on their antioxidant and anti-inflammatory properties. Here, we performed a systematic review of clinical trials analyzing the efficacy of n-3 PUFA on both systemic oxidative stress and on NAFLD/NASH features in adults. As a matter of fact, it remains controversial whether n-3 PUFA are effective to counteract oxidative stress. On the other hand, data suggest that n-3 PUFA supplementation may be effective in the early stages of NAFLD, but not in patients with more severe NAFLD or NASH. Future perspectives and relevant aspects that should be considered when planning new randomized controlled trials are also discussed.
\end{abstract}

Keywords: oxidative stress; non-alcoholic fatty liver disease; aging; omega-3 fatty acids

\section{Introduction}

\subsection{Aging and Oxidative Stress}

Aging is a gradual decline of physiological function with age [1] accompanying the intrinsic, inevitable and irreversible age-related process of loss of viability and increase in vulnerability in human beings [2]. Aging is a ubiquitous complex phenomenon as a consequence of the interaction of genetic, epigenetic, environmental, and stochastic factors throughout life. During this multifactorial process, the damages occurring in molecules, cells, and tissues gradually accumulate in a timely manner. The 
genetic predisposition and epigenetic modifications afterward have mostly determined the acceleration or the prevention of aging and its related pathophysiological functions at a whole organism level. General statistics have shown that as each individual reaches the mid-age of their biological lifespan, numerous subsequent malign, pathological, and deteriorative consequences start to interfere with the cardiovascular system, metabolic processes, neurodegenerative disorders, muscle and vision functions, among others [3-6].

Reactive oxygen species (ROS) are generated within the cells by cellular metabolic activities such as cell survival, stressor responses, inflammation [7,8], and environmental factors, such as air pollutants or cigarette smoke [9]. Increased ROS production has been recognized as a critical contributor to aging since the middle 50's when D. Harman hypothesized "The free Radical Theory of Aging" [10] This theory proposed that highly unstable and reactive molecules generated by cellular metabolism or environmental factors cannot be removed or neutralized, which produce proteins, lipids, and DNA damage, eventually leading to a defective function of the organelles in aged subjects. Presently it is known that during the final third of the lifespan, aged animals and humans have lower adaptive homeostatic capabilities [11,12] mainly caused by an impaired antioxidant defense system, which in turn promotes the accumulation of oxidative stress-induced molecular damage and triggers cellular senescence [11]. Oxidative stress can be evaluated by several biomarkers. Hydrogen peroxide, superoxide radical, oxidized glutathione (GSSG), carbonyls, and nitrotyrosine can be easily measured from plasma as biomarkers of oxidation [9]. Lipid peroxidation refers to the oxidative degradation of lipids generally located in biological membranes including phospholipids and cholesterol which propagates free radicals [13]. Quantifying the secondary breakdown products can indicate in vivo lipid peroxidation. Several biomarkers are identified for this: 4-hydroxynonenal (4-HNE), 4-hydroxyhexenal (4-HHE), malondialdehyde (MDA), F2-isoprostanes, $\alpha$-tocopherol concentration, plasma thiobarbituric acid-reactive substances (TBARS) level, total reactive antioxidant potential (TRAP), superoxide dismutase (SOD) activity and low density lipoprotein (LDL) oxidative susceptibility [13]. Plasma and urine F2-isoprostanes have been identified as excellent and sensitive biomarkers of in vivo lipid peroxidative damage $[13,14]$. These compounds are formed in a free radical-dependent manner in cell membranes at the site of free radical attack from arachidonic acid [14]. Their production has been reported to be altered in many syndromes putatively associated with oxidative stress [15]. Moreover, human aging has been characterized as a chronic, low-grade inflammation state, widely named as "inflammaging" [16]. Thus, chronic cell oxidative stress activates a pro-inflammatory program leading to acquisition of the senescence-associated secretory phenotype (SASP) characterized by the increased secretion of pro-inflammatory factors involving the secretion of soluble factors (interleukins, chemokines and growth factors), degradative enzymes such as matrix metalloproteases (MMPs) and insoluble proteins/extracellular matrix (ECM) components. Inflammaging, caused by an accumulation of senescent cells exhibiting SASP in tissues, is considered a risk factor for the development of most age-related diseases and therefore, for morbidity and mortality in the elderly [17].

\subsection{Aging and the Pathophysiology of Non-Alcoholic Fatty Liver Disease (NAFLD)}

\subsubsection{Concept and Pathogenesis of NAFLD}

Liver function and structure impairment is one of the predominant hallmarks of aging [18]. NAFLD is considered to be the consequence of excessive accumulation of triglycerides (TG) in the cytoplasm of hepatocytes $(>0.5 \%$ ) without the over-consumption of alcohol in order to compensate for the increased cellular content of non-esterified fatty acids (free fatty acids; FFAs) [19]. NAFLD is highly prevalent worldwide [20] and estimated to be globally around $24 \%$ according to data published in 2016 [20]. NAFLD is the most frequent cause of chronic liver disease in western countries [16,21,22], encompassing a broad spectrum of physio-pathological conditions from simple steatosis to non-alcoholic steatohepatitis (NASH). According to accepted clinical criteria [23], NASH should only be established when hepatocyte fat storage is accompanied with lobular inflammation 
and ballooned hepatocytes, where the grade of fibrosis sharpens progressively by stage. If no proper therapeutic approach is implemented, NASH can ultimately progress to hepatic cirrhosis or hepatic carcinoma where the scar tissue becomes irreversible, the liver losses its functions partly or completely, or even develop to hepatocellular carcinoma [24]. NAFLD can trigger or manifest other cardiometabolic disorders including obesity, disrupted glucose and lipid metabolism, insulin resistance (IR) and type 2 Diabetes Mellitus [25-28]. Aging is also the most common cause for the progression of NAFLD. Indeed NAFLD is commonly found in the elderly, but the results from the Rotterdam study suggested that the prevalence of NAFLD decreased with advancing age, suggesting a positive selection of elderly without NAFLD [29]. Finally, regarding aging and NAFLD, it is important to note that the effect of aging is different between men and women because women experience many physical changes associated with menopause during aging. Although controversial results have been found on the direct cause or on the mechanisms involved, there is increasing evidence indicating that post-menopausal women are more susceptible than men to develop NAFLD [30]. The study of Klair et al. [31] showed that time from menopause is directly associated with an increased likelihood of having more severe NAFLD [31]. In support of this hypothesis, studies in rodents suggested that these observed deleterious aging-related effects on females' livers might be attributed to impaired lipid metabolism. In fact, in these models, estrogens increased fatty acid $\beta$-oxidation by AMPK activation [32,33]. Taking together these studies, it could be speculated that in menopausal women, lower serum estrogen levels might constitute a major cause for impaired lipid accumulation/break down balance and therefore for the promotion of NAFLD development. However, contrarily, the study of Veronese et al. [34] described that the years from menopause are not associated with the severity of NAFLD. Moreover, the study found that adiposity and metabolic syndrome features were associated with higher liver steatosis levels, concluding that not menopause itself but the menopause-associated increased adiposity (particularly the excessive accumulation of abdominal adiposity) is the major cause for NAFLD development in post-menopausal women [34].

However, the pathogenesis of liver disease during aging remains inaccurately defined. Nonetheless, aging is accompanied by a gradual decrease of hepatic blood flow and liver volume ranging from $20 \%$ to $40 \%[18,35,36]$. In addition to understanding the nature of these changes, few studies conducted in humans have investigated cholesterol levels. Studies in humans have shown a decrease in low-density lipoprotein by $35 \%[37,38]$ and an increase in biliary cholesterol output, possibly related to a decrease in cholesterol degradation to bile acid [39].

NAFLD has a complex pathophysiology with multiple manifestations/complications. Liver steatosis arises as a consequence of an imbalance between hepatic lipid accumulation (from accelerated FFA influx and de novo lipid synthesis) and hepatic lipid clearance (free fatty acid oxidation (FAO) and very low-density lipoprotein (VLDL) excretion) [40]. In terms of pathogenesis of NAFLD, a "two hits hypothesis" was proposed over two decades ago [41] to make an approach to unravel the progression from NAFLD to NASH. The "first hit" core is peripheral IR as a leading cause accompanying obesity and metabolic syndrome. In adipose tissue (mainly visceral depot), IR causes an increase in lipolysis, and consequently the delivery of FFA into the liver increases and TG accumulation occurs. Moreover, de novo lipogenesis (DNL) plays a pivotal role in FFA synthesis, and the excess of carbohydrates is converted into fatty acids and esterified into TG [42]. In contrast, the export of FFA and TG is decreased, while the beta-oxidation of mitochondrial long-chain fatty acids is increased. Indeed, it has been proposed that hepatic TG accumulation is also probably a consequence of saturation of FFA oxidation and VLDL) secretion, since both of these pathways are up-regulated rather than decreased in patients with NAFLD [43]. However, several studies have suggested a protective effect of TG [44,45] and that an increased level of intrahepatic TG may be a biomarker instead of a cause factor of IR [46].

A "second hit" seems to be needed to develop NASH from NAFLD, such as oxidative stress, which in terms may explain the progression to liver fibrosis. An imbalanced production of reactive nitrogen species (RNS) or ROS and the antioxidant molecules of the organism produces oxidative stress, which can induce hepatocellular injury by the inhibition of the mitochondrial respiratory chain 
enzymes and the inactivation of both glyceraldehyde-3-phosphate dehydrogenase and membrane sodium channels. Furthermore, ROS cause lipid peroxidation and cytokine production, contributing to hepatocellular injury and fibrosis [47], and promote the progression from simple steatosis to NASH [48]. Furthermore, ROS induce the directional migration of resident hepatic pro-fibrogenic cells, resulting in liver fibrosis [49].

In the year 2010, Tilg and Moschen [50] proposed a multiple parallel hits model, suggesting that many hits may act in parallel favoring the progression from NAFLD to NASH, finally resulting in liver inflammation and endoplasmic reticulum (ER) stress, and that gut and adipose tissue derived factors may play a central role (Figure 1).

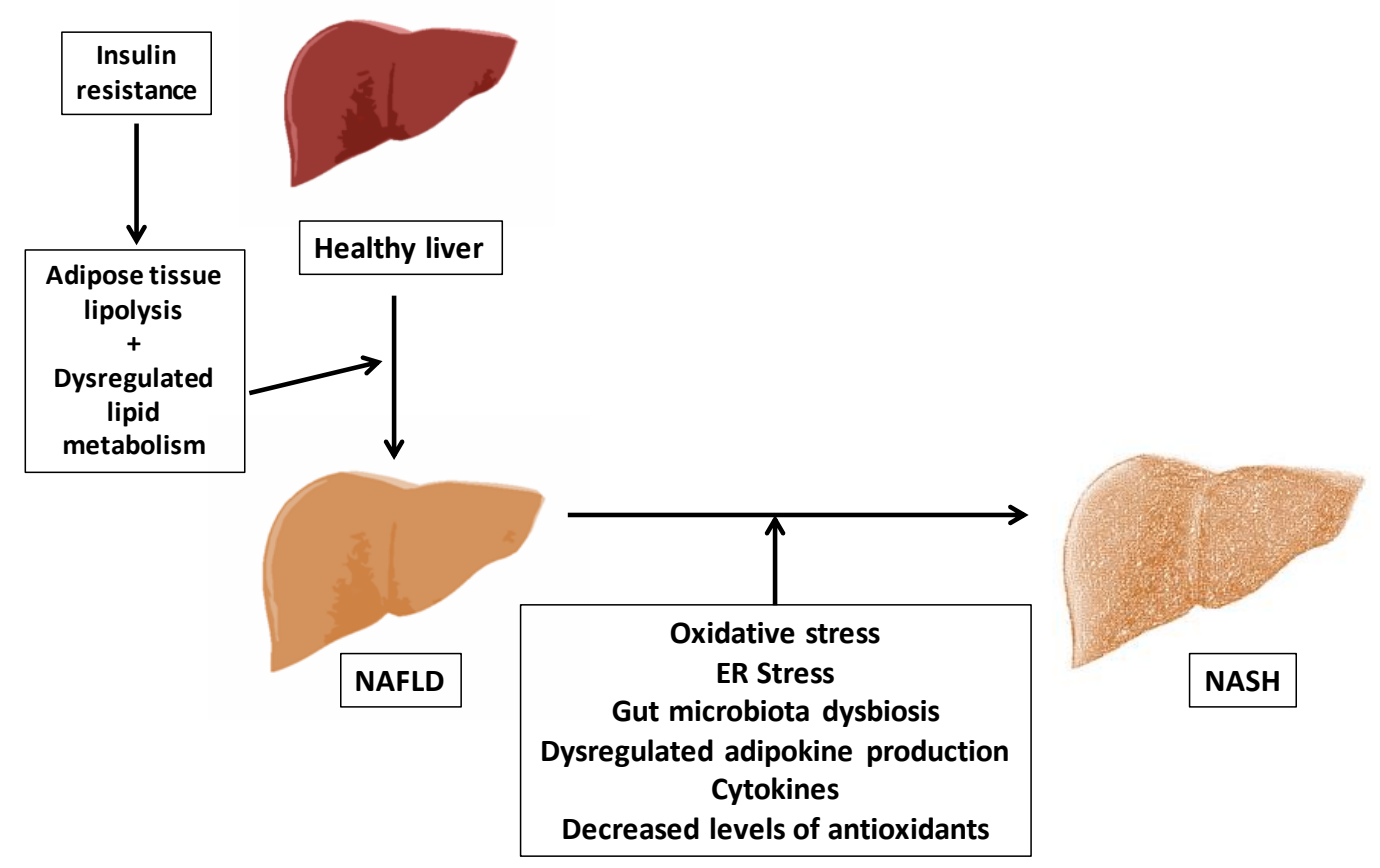

Figure 1. Multiple parallel hits NAFLD pathogenesis model. Non-alcoholic fatty liver disease (NAFLD). Non-alcoholic steatohepatitis (NASH). Endoplasmic reticulum (ER).

Lipotoxicity seems to play a key role in the pathophysiology of NASH. Lipotoxic injury is also triggered by an excessive FFA flux, especially of saturated fatty acids (SFAs), rather than due to simple TG accumulation. The excess of FFA facilitates the generation of lipotoxic metabolites (such as ceramides, diacylglycerol, lysophosphatidyl choline and ROS) contributing also to the development of liver oxidative stress [43]. Moreover, in NAFLD and aging, increased visceral adipose tissue is associated with increased infiltration of M1 macrophages, which triggers adipose tissue IR and inflammation and leads to a disturbed adipokine profile (low adiponectin, an anti-inflammatory adipokine with beneficial effects in NAFLD; and high levels of pro-inflammatory adipokines such as leptin, IL-1 $\beta$, IL-6 and tumor necrosis factor- $\alpha$; TNF- $\alpha$ ), which finally induces liver inflammation and hepatic IR, as previously stated [51]. Therefore, it is clear that signals and hormones derived from the adipose tissue beyond toxic lipids might play a central role in NAFLD/NASH.

In the crosstalk between the gut and the liver, the modifications in the microbiota are considered to have major influence in NASH progression [50]. The human gut is colonized by at least 100 trillion of microorganisms (gut microbiota) that maintain symbiotic relationships with the host. Several lines of evidence have demonstrated strong relationships between changes in gut microbiota composition and the etiology of obesity, inflammation, type 2 diabetes and NAFLD [52]. A major mechanism linking gut dysbiosis with the progression of chronic liver diseases is the translocation of bacteria or bacterial products into the portal circulation [53]. The gut bacteria may contribute to NAFLD by producing bacteria-derived endotoxins (lipopolysaccharide, LPS). It has been shown that feeding 
with a high-fat or a high-carbohydrate diet causes elevated levels of circulating endotoxin [54], which may also affect the accumulation of hepatic fat [50]. The gut microbiota also produces microbial metabolites such as short-chain fatty acids (SCFAs) which play a critical role in regulating host energy harvest [55]. SCFAs have anti-inflammatory properties, can directly act as lipid precursors in the liver, and may act by interacting with the G protein- coupled receptor 43 (Gpr43) [56]. An altered microbiota also inhibits the synthesis of fasting-induced adipocyte factor (FIAF; also known as angiopoietin-related protein 4, ANGPTL4), which has been associated with a higher accumulation of lipids in the liver [53]. Moreover, the endogenous ethanol produced by the intestinal microbiota favors the transport of endotoxins in the gut vessels [57]. It has been observed that patients with NASH have higher abundance of ethanol-producing bacteria in their gut microbiome, suggesting a potential involvement of alcohol-producing gut microbiota in NASH progression [58]. Ethanol and its derived compounds (acetaldehyde and acetate) [59] induce the formation of ROS by hepatic stellate cells and Kupffer cells [60]. Jones and Neish [61] suggested that the interaction between the gut epithelia and some groups of enteric commensal bacteria induces rapid generation of ROS within host cells [61], and together with LPS, ROS promote increased TLR4 gene expression [62]. Recent data have shown that the gut microbiota regulate the metabolism of the major intracellular antioxidant, glutathione (GSH), in the host organism [63]. Thus, lower levels of GSH can contribute to oxidative stress [64,65].

In conclusion, this multiple parallel hits model reflects more deeply the phenomena that contribute to the pathogenesis of NAFLD/NASH than the previous two hits model. Moreover, aging is an important determinant for NAFLD/NASH development. All the previously described multifactorial mechanisms involved in the pathogenesis of NAFLD/NASH (IR, increased visceral adiposity, inflammation, oxidative and ER stress, dysbiosis) are accentuated during aging, which could be underlying the higher prevalence of these pathologies among elderly. Thus, several therapeutic approaches can be merged from targeting these key factors for the promotion of liver health in the context of aging.

\subsubsection{Genetic Variances Susceptibility for NASH and Oxidative Stress}

NAFLD is also considered as a poligenic disease [66] where different polymorphisms can affect the three main pathways: genes that participate in TG accumulation, in inflammatory processes and that are part of the oxidative-stress pathway. (i) Genes that participate in the accumulation of triglycerides such as: patatin-like phospholipase domain containing protein 3 (PNPLA3) and transmembrane 6 superfamily member 2 (TM6SF2) [67]. PNPLA3 rs738409 polymorphism has been recently associated through a meta-analysis with NAFLD susceptibility and also to other aggressive diseases [68]. In addition, Li et al. [69] have found a significant association of TM6SF2 rs58542926 polymorphism and the risk of NAFLD. (ii) Genes involved in the inflammatory processes: TNF, IL-6 and toll-like receptor 4 (TLR4) [66]. TNF-238 [70,71] and TNF-308 [72] are the main polymorphisms of TNF related with the disease, although their roles are still under consideration. -174 polymorphism in the IL-6 gene promoter region has been shown to be more prevalent in NAFLD patients than in healthy subjects [71,72]. In the case of TLR4, -299 polymorphism seems to be preventive [73], although a polymorphism (-159) in its co-receptor CD14 has been associated with an increased risk of NAFLD [74]. (iii) Genes that are part of the oxidative-stress pathways: SOD2 gene (mitochondrial enzyme manganese-dependent superoxide dismutase (MnSOD) is encoded by this gene), uncoupling protein 3 (UCP3) and glutamate-cystein ligase (GCLC) [66]. C47T polymorphism in the SOD2 gene has been associated with NASH in obese children [75] and with NAFLD fibrosis severity [76]. A UCP3 single nucleotide polymorphism (SNP) has been related with NAFLD in Chinese children [77] and in obese adults [78]. Furthermore, the genetic variant -129C/T in the GCLC gene has been associated with NASH $[79,80]$.

\subsubsection{The Diagnosis of NAFLD}

The diagnosis of NAFLD is often based on the following criteria: non-alcoholic, detection of steatosis either by imaging or by histology and an appropriate exclusion of other liver diseases [27,81-83]. 
Daily alcohol consumption approximately below $30 \mathrm{~g}$ in men and $20 \mathrm{~g}$ in women can be included in the target population according to conducted epidemiological studies [23], but no consensus of the exact quantity has been reached. Ultrasonography (US) and magnetic resonance imaging (MRI) are currently the most employed methods to diagnose NAFLD, since they are non-invasive, comparatively low cost, and commonly available, with a sensitivity of $89 \%$ and $77 \%$, and a specificity of $93 \%$ and $89 \%$ in diagnosing liver steatosis and liver steatohepatitis respectively $[84,85]$. However, the US is subjective and operator-dependent and not that good at assessing liver steatosis, while the MRI is more objective and better at quantifying steatosis [23]. Liver biopsy is considered to be the "gold" standard for identifying steatohepatitis (NASH) by correlating histological features [86]. Although liver biopsy is the definitive tool to diagnose NASH, it lacks availability being an expensive and invasive procedure, and it would not be necessary to apply since the prevalence of liver steatosis is much more frequent than NASH. Recently, several non-invasive, simple diagnostic indices have shown their utility in diagnosing NAFLD and NASH, since they are calculated based on anthropometric data and biochemical analysis (Table 1). These indices include: the Fatty liver index (FLI) [87,88], the hepatic steatosis index (HSI) [89] and the ZJU (Zhejiang University) index [90]. Other non-invasive scores are the NAFLD fibrosis score [91-93] the BARD score [91] and the FIB-4 index [94]. More research is needed to identify and confirm truly independent and quantitative markers of steatosis. 
Table 1. NAFLD index and their hallmarks.

\begin{tabular}{|c|c|c|c|}
\hline NAFLD Index & Predictors & Hallmarks & Interpretation \\
\hline Fatty liver index (FLI) & $\begin{array}{l}\text { Fatty Liver Index }(\mathrm{FLI})=\mathrm{e}^{\mathrm{y}} /\left(1+\mathrm{e}^{\mathrm{y}}\right) \times 100 \\
\text { Where } \mathrm{y}=0.953 \times \ln (\text { triglycerides, } \mathrm{mg} / \mathrm{dL}) \\
+0.139 \times \mathrm{BMI}, \mathrm{kg} / \mathrm{m}^{2}+0.718 \times \ln (\mathrm{GGT} \\
\mathrm{U} / \mathrm{L})+0.053 \times \mathrm{WC}, \mathrm{cm}-15.745)[95]\end{array}$ & $\begin{array}{l}\text { Identified NAFLD and the optimal } \\
\text { cut-off point with accuracy. }\end{array}$ & $\begin{array}{l}\text { FLI }<30 \text {, no FL (with a negative likelihood ratio of up to } 0.2 \text { ); } \\
\qquad 60<\text { FLI }<30 \text {, Inconclusive } \\
\text { FLI } \geq 6 \text { 60, FL present (with a likelihood ratio starting from } 4.3 \text { ) }\end{array}$ \\
\hline $\begin{array}{l}\text { Lipid accumulation product } \\
\text { (LAP) }\end{array}$ & $\begin{array}{l}\text { LAP for men }=(\mathrm{WC}[\mathrm{cm}]-65) \times(\mathrm{TG} \\
\text { concentration }[\mathrm{mmol} / \mathrm{L}]) \\
\text { LAP for women }=(\mathrm{WC}[\mathrm{cm}]-58) \times(\mathrm{TG} \\
\text { concentration }[\mathrm{mmol} / \mathrm{L}])[96]\end{array}$ & $\begin{array}{l}\text { Associated with the presence and } \\
\text { severity of NAFLD, among young } \\
\text { and aged population [97,98]. NOT } \\
\text { able to predict liver fat content [99]. }\end{array}$ & $\begin{array}{c}\text { The optimal cut-off value for LAP was } 31.6 \text { with sensitivity of } \\
88 \%(95 \% \text { CI, } 77-96 \%) \text {, specificity of } 82 \%(95 \% \text { CI, } 76-87 \%) \text { for } \\
\text { males and with a sensitivity of } 66 \%(95 \% \text { CI, } 52-78 \%), \\
\text { specificity of } 93 \%(95 \% \text { CI, } 88-96 \%) \text { for females. }\end{array}$ \\
\hline Hepatic steatosis index (HSI) & $\begin{array}{l}\text { Hepatic steatosis index }(\mathrm{HSI})=8 \times \\
\text { (ALT/AST ratio) }+ \text { BMI }(+2, \text { if female; }+2, \text { if } \\
\text { diabetes mellitus) [89]. }\end{array}$ & $\begin{array}{l}\text { A simple, efficient screening tool } \\
\text { for NAFLD, used for selecting } \\
\text { individuals for liver } \\
\text { ultrasonography [89]. }\end{array}$ & $\begin{array}{c}\text { At values of }<30.0 \text { or }>36.0 \text {, HSI ruled out NAFLD with a } \\
\text { sensitivity of } 93.1 \% \text {, or detected NAFLD with a specificity of } \\
92.4 \% \text {, respectively [89]. }\end{array}$ \\
\hline $\begin{array}{l}\text { The ZJU (Zhejiang University) } \\
\text { index }\end{array}$ & $\begin{array}{l}\text { ZJU index }=\text { BMI }\left(\mathrm{Kg} / \mathrm{m}^{2}\right)+\text { FPG }(\mathrm{mmol} / \mathrm{L}) \\
+ \text { TG }(\mathrm{mmol} / \mathrm{L})+3 \times \text { ALT }(\mathrm{IU} / \mathrm{L}) / \mathrm{AST} \\
(\mathrm{IU} / \mathrm{L}) \text { ratio }(+2, \text { if female })[90]\end{array}$ & $\begin{array}{l}\text { Confirmed to have significance in } \\
\text { terms of diagnosing NAFLD [90]. }\end{array}$ & $\begin{array}{l}\text { At a value of }<32.0 \text {, the ZJU index could rule out NAFLD } \\
\text { with a sensitivity of } 92.2 \% \text {, and at a value of }>38.0, \text { the ZJU } \\
\text { index could detect NAFLD with a specificity of } 93.4 \%[90] \text {. }\end{array}$ \\
\hline NAFLD fibrosis score & $\begin{array}{l}\text { NAFLD Score }=-1.675+(0.037 \times \text { age } \\
[\text { years }])+\left(0.094 \times \mathrm{BMI}\left[\mathrm{kg} / \mathrm{m}^{2}\right]\right)+(1.13 \times \\
\text { IFG/diabetes }[y e s=1, \text { no }=0])+(0.99 \times \\
\text { AST/ALT ratio })-(0.013 \times \text { platelet count } \\
[\times 109 / \mathrm{L}])-(0.66 \times \text { albumin }[\mathrm{g} / \mathrm{dL}])[92]\end{array}$ & $\begin{array}{l}\text { Identifies patients without severe } \\
\text { fibrosis, comparatively more } \\
\text { difficult to estimate [91]. }\end{array}$ & $\begin{array}{l}\text { Low cut-off score ( }-1.455) \text { : advanced fibrosis ruled out with } \\
\text { high accuracy (negative predictive value of } 93 \% \text { and } 88 \% \text { in } \\
\text { the estimation and validation groups, respectively). High } \\
\text { cut-off score ( } 0.676) \text {, advanced fibrosis diagnosed with high } \\
\text { accuracy (positive predictive value of } 90 \% \text { and } 82 \% \text { in the } \\
\text { estimation and validation groups, respectively) [92]. }\end{array}$ \\
\hline BARD score & $\begin{array}{c}\text { Based on AST/ALT ratio, presence of } \\
\text { diabetes and BMI [100]. }\end{array}$ & $\begin{array}{l}\text { Identifies patients without severe } \\
\text { fibrosis, but easier to estimate and } \\
\text { does not have indeterminate } \\
\text { results [91]. }\end{array}$ & $\begin{array}{c}\text { BMI } \geq 28=1 \text { point, } \mathrm{AAR} \text { of } \geq 0.8=2 \text { points, } \mathrm{DM}=1 \text { point } \\
\text { A score of } 2-4 \text { was associated with an OR for advanced } \\
\text { fibrosis of } 17 \text { (confidence interval } 9.2 \text { to } 31.9 \text { ) and a negative } \\
\text { predictive value of } 96 \% \text { [100]. }\end{array}$ \\
\hline FIB-4 index & $\begin{array}{c}\text { FIB-4 Score }=\text { age }([\mathrm{yr}] \times \text { AST }[\mathrm{U} / \mathrm{L}]) /((\mathrm{PLT} \\
\left.\left.\left[10^{9} / \mathrm{L}\right]\right) \times(\mathrm{ALT}[\mathrm{U} / \mathrm{L}])^{1 / 2}\right)[101]\end{array}$ & $\begin{array}{l}\text { In patients }<35 \text { or }>65 \text { years old, } \\
\text { the score has been shown to be less } \\
\text { reliable }[94,101] \text {. }\end{array}$ & $\begin{array}{l}\text { At a cut-off of }<1.45 \text { in the validation set, the negative } \\
\text { predictive value to exclude advanced fibrosis (stage } 4-6 \text { ) was } \\
90 \% \text { with a sensitivity of } 70 \% \text {. A cut-off of }>3.25 \text { had a } \\
\text { positive predictive value of } 65 \% \text { and a specificity of } 97 \% \text {. } \\
\text { Using these cut-offs, } 87 \% \text { of the } 198 \text { patients with FIB- } 4 \text { values } \\
\text { outside } 1.45-3.25 \text { would be correctly classified [101]. }\end{array}$ \\
\hline
\end{tabular}

GGT, gamma-glutamyl transpeptidase; OR, odd ratio; WC, waist circumference. 


\section{Nutrients, Oxidative Stress and NAFLD/NASH}

Considering the role of oxidative stress in the progression of aging and especially in the risk of developing aging-related pathologies, it is important for researchers to look for potential therapeutic targets that might delay the progression of oxidative damage in the early stages of aging. For this reason, increasing attention has been paid to the role that certain dietary nutrients may play in oxidative stress. Oxidative stress has been identified as a key factor associating obesity with related disorders such as cardiovascular diseases or type 2 diabetes mellitus, which can be triggered by a high-level consumption of several macronutrients: glucose, SFAs or omega- 6 polyunsaturated fatty acids (n-6 PUFA) inducing inflammation through nuclear factor kappa-light-chain-enhancer of activated B cells (NF- $\mathrm{BB}$ ) mediated pathways [102]. Contrariwise a large body of research has investigated the potential beneficial effect of dietary antioxidants such as vitamin A, vitamin E, vitamin C, selenium, $\alpha$-lipoic acid, resveratrol and other polyphenols in the prevention of metabolic and age-related chronic diseases [103-105].

According to previous studies, omega-3 polyunsaturated fatty acids (n-3 PUFA) may exert a protective effect on cardiovascular and metabolic diseases, which have been related to its antioxidant and anti-inflammatory properties [106-108]. Richard et al. [109] reported that n-3 PUFA may indirectly act as antioxidants by lowering ROS production and superoxide scavenging. Petinelli et al. [110] found that the liver of NAFLD patients exhibits a marked enhancement in n-6 PUFA/n-3 PUFA ratio, which may favor lipid synthesis over oxidation and secretion, leading to steatosis. In this context, n-3 PUFA have been involved in the regulation of the metabolic switch from anabolism (lipogenesis) to catabolism (FAO) by inhibiting Sterol regulatory element-binding protein 1 (SREBP1c) and activating peroxisome proliferator-activated receptor alpha (PPAR $\alpha)$, a positive regulator of FAO [110-112]. However, it remains the theoretical concern that the many double bonds of eicosapentaenoic acid (EPA) and docosahexaenoic acid (DHA) may lead to an increased unsaturation index once they are incorporated into the membranes and lipoproteins [113]. This situation could induce higher lipid peroxidation based on the premise that fatty acid oxidizability might be directly associated with the number of double bonds in the fatty acid chain [114]. However, some in vitro and in vivo studies have challenged these theoretical considerations. Indeed, n-3 PUFA may also alleviate IR, lipid accumulation, pro-inflammatory actions, and ROS production, and promote the FAO in NAFLD, in part by modulating the production of bioactive adipokines (leptin and adiponectin) that control the crosstalk between adipose tissue and key metabolic organs such as the liver and muscle [115].

On the other hand, understanding that nutrient intake can also promote or reduce the pro-inflammatory response and oxidative stress occurring in NAFLD and NASH is of crucial importance in order to prevent the development and/or severity of these pathologies [116]. Few studies have suggested that over-consumption of carbohydrate-enriched drinks, which contain elevated levels of fructose increased SREBP1c and downstream fatty acid synthesis genes, resulting in reduced liver insulin signaling [117]. Meanwhile, fructose promotes inflammation by modelling inflammatory genes [118-120], and down-regulates the hepatic mitochondria beta-oxidation [121]. Overloaded iron levels and decreased copper level have been suggested to play a role in NASH development, inducing inflammation and oxidative stress through several cellular mechanisms [122-124]. Increased dietary intake of SFAs, cholesterol and trans-fat induces DNL leading to ER stress and apoptosis [125]. These nutrients are mostly in favor of the NASH development since they trigger inflammation and ER stress.

In conclusion, increased fructose, SFA, cholesterol, trans-fat, iron intake and decreased copper intake can induce oxidative stress in NAFLD; on the contrary, nutrient intake such as n-3 PUFA, vitamin $A$, vitamin $E$, vitamin $C$, selenium, $\alpha$-lipoic acid, resveratrol, and other polyphenols may ameliorate oxidative stress and NAFLD. To our knowledge, there are not systematic reviews analyzing the main outcomes of randomized controlled trials aimed to characterize the actions of n-3 PUFA supplementation on oxidative-stress biomarkers.

On the other hand, although several trials and recent meta-analysis have suggested that n-3 PUFA may have beneficial effects on NAFLD patients, the outcomes on different biomarkers of fatty liver 
disease (AST, ALT, GGT or liver fat content by US) are sometimes heterogeneous [126-130]. Moreover, recent randomized controlled trials have produced conflicting data in NAFLD/NASH patients [131].

Based on these premises, the aims of the current study are to perform a systematic review of the current clinical trials analyzing the potential beneficial effects of n-3 PUFA supplementation on systemic oxidative stress as well as their efficacy in the treatment of NAFLD and NASH in adult patients.

\section{3. n-3 PUFA in Oxidative Stress and NAFLD/NASH}

\section{1. n-3 PUFA (Dietary Sources and Metabolism)}

$\alpha$-linolenic acid (ALA) and linoleic acid (LA) are essential PUFA obtained from diet, since they cannot be synthesized in humans [26,27]. LA is the major n-6 PUFA, and ALA is an n-3 PUFA [90]. n-6 PUFA can be obtained mainly from poultry, eggs, nuts, sesame seeds, dairy products, and lower amounts in grains and seeds [26,27]. ALA (18:3 n-3) can be found in flaxseed, canola and soybean oils, green leafy vegetables and walnuts [132]. In the body, LA is metabolized to arachidonic acid (AA; 20:4 n-6), and ALA is metabolized to EPA (20:5 n-3) and DHA (22:6n-3) [102]. The conversion of ALA to EPA is limited; however, some studies show that ALA can be converted to EPA when adequate intake of ALA is ensured and low intake of n-6 PUFA happens. Moreover, in vivo the conversion of ALA to DHA has been reported to be even more limited [133]. Regular intake of EPA and DHA from our diet is needed to guarantee an optimal supply of n-3 PUFA. EPA and DHA are acquired mainly from marine products: fatty fishes such as salmon, tuna, and sardines, which store fatty acids throughout their body, and lean fishes such as cod and hake that store fatty acids in the liver [134]. The common minimal recommended daily intake of n-3 PUFA ranges approximately from 0.35 to $0.40 \mathrm{~g}$ per day ( $0.5 \%$ of total fat) [135]. The American Heart Association guidelines suggests a two-portion of fatty fish intake per week to prevent hypertriglyceridemia and cardiovascular disease [136]. Yet, no consensus of the precise amount has been reached. When humans consume EPA and DHA, they partially replace the n-6 PUFA (particularly AA) in cell membranes, especially those of platelets, erythrocytes, neutrophils, monocytes, and liver cells [137]. As a result, ingestion of EPA and DHA from fish or fish oil ameliorates the pro-inflammatory, atherogenic pro-aggregatory and pro-thrombotic effects caused by n-6 PUFA, which leads to down regulation of pro-inflammatory metabolites such as prostaglandin E2, thromboxane A2 and leukotriene B4 [100,138,139].

Western diets usually contain higher amounts of n-6 PUFA and lower amounts of n-3 PUFA (n-6/n-3 PUFA ratio around 15:1). Current evidence suggests that it is imperative to maintain an optimal n-6/n-3 PUFA ratio [140-142], since this not only plays a role in the pathogenesis of CVD, but also has an impact on cancer, inflammatory and autoimmune diseases [140]. A suggested ratio of n-6/n-3 PUFA is 3-4:1 to maintain a pro-inflammatory/anti-inflammatory equilibrium in the organism [143,144]. The increase in the n-6/n-3 PUFA ratio can also exacerbate the risk of obesity and NAFLD [145-147].

EPA and DHA have been demonstrated to serve as substrates for the formation of a novel series of specialized pro-resolving lipid mediators (SPMs). These SPMs include: EPA derived E-series Resolvins (RvE-1), DHA derived D-series Resolvins (RvD1-6), protectins (NPD1, PDX) and Maresin (MaR1-2) [148]. Studies have demonstrated their potent anti-inflammatory and pro-resolutive properties acting at doses much lower than their n-3 PUFA precursors [108]. Liver inflammation can be resolved by a shift to M2 macrophages, and these SPMs can act as 'stop signals' of the inflammatory response and promote liver regeneration [149]. In this way, several studies in obese mice with IR and liver steatosis have found that treatment with some of these SPMs including RvE1, RvD1, 17-HDHA and Maresin 1 decrease adipose tissue and liver inflammation and significantly improve IR and reduce liver steatosis [150-154]. Moreover, n-3 PUFA supplementation from marine sources to obese mice promotes the synthesis of n-3 PUFA-derived SPMs in liver and adipose tissue, ameliorating tissue inflammation and peripheral inflammation and IR [155-157]. Interestingly, a clinical trial conducted in humans has shown that short-term n-3 PUFA supplementation for 5 days results in concentrations of SPMs that are biologically active in healthy humans [153]. Other trials in severely obese subjects have demonstrated that the 
production of anti-inflammatory-pro-resolving lipid mediators in adipose tissue is enhanced after $n-3$ PUFA treatment [158]. All these findings suggest that the beneficial metabolic effects attributed to n-3 PUFA can be partly mediated by the production of these SPMs in key metabolic tissue such as liver and adipose tissue.

\section{2. n-3 PUFA and Oxidative Stress}

To perform a review about the effects of n-3 PUFA supplementation on oxidative-stress biomarkers in adults, potentially relevant studies were retrieved by a systematic search in the PubMed database. The search was performed using the terms "n-3 PUFA and oxidative stress". Around 1525 non-duplicated entries were found. Titles and abstracts were reviewed for the first selection, and full texts were checked only when abstracts itself were not able to reveal the nature of the studies. The filters applied were clinical trial, free full text, human, adults with cardiometabolic disorders, chronic inflammatory diseases, or healthy adults. Twenty-seven studies were included after two selections (Figure 2).

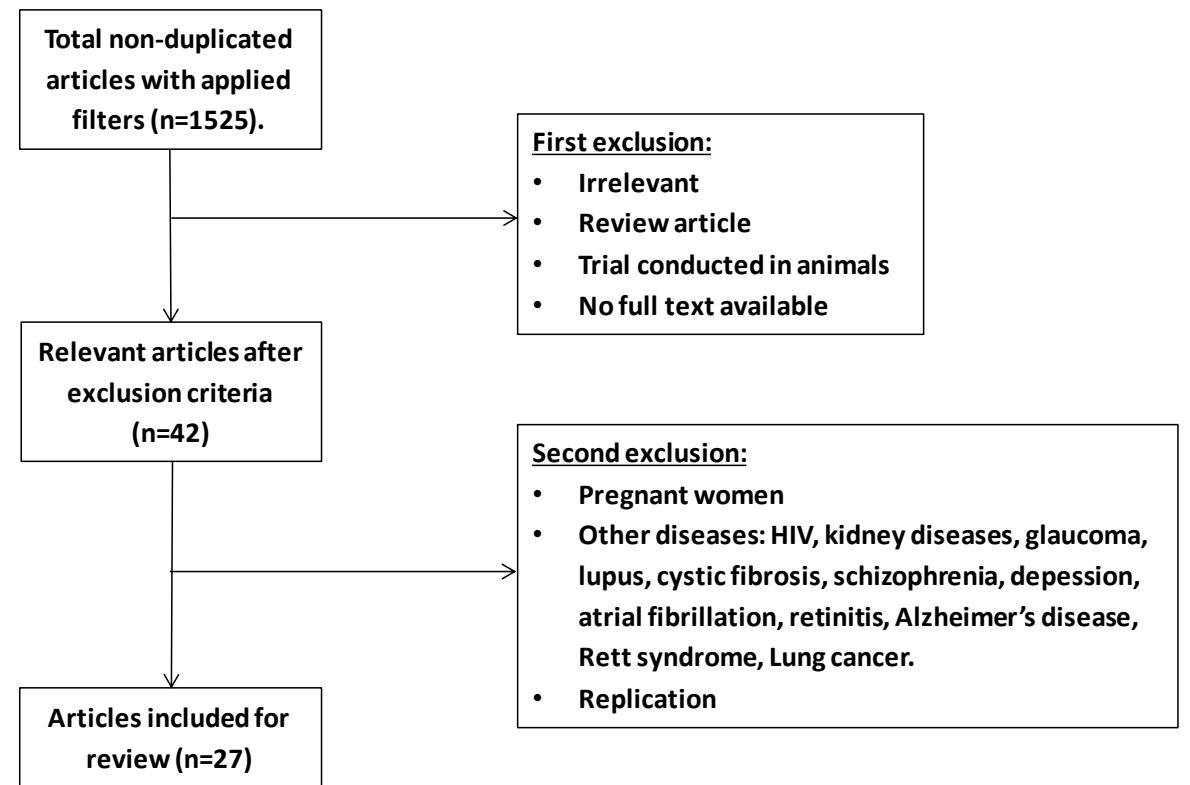

Figure 2. Flowchart of selection process based on n-3 PUFA and oxidative stress.

Table 2 summarizes the characteristics of the trials (population, design, intervention) and the main outcomes observed in biomarkers of oxidative stress after n-3 PUFA supplementation in adults.

The studies described in Table 2 include different populations as healthy and overweight/obese adults, patients of both genders with cardiometabolic disorders, metabolic syndrome, T2DM, dyslipidemia, hypertriglyceridemia, with a wide age range. Moreover, doses and supplementation periods as well as the oxidative-stress biomarkers assessed (including urine F2-isoprostanes, 4- $\mathrm{HHE}$ and 4-HNE, oxidized LDL, plasma $\alpha$-tocopherol, and enzymatic activity of Glutathione reductase (GR), Glutathione peroxidase (GPx), and Catalase (CAT) vary from study to study. Regarding the main outcomes, several studies showed a lowering effect on oxidative stress and lipid peroxidation biomarkers with EPA or DHA supplementation [159-169]. However, other studies showed increased levels of oxidative stress or lipid peroxidation biomarkers [170-175] or no changes [176-183]. Other studies have observed divergent effects of n-3 PUFA supplementation on different oxidative-stress biomarkers [184,185]. 
Table 2. Effects of n-3 PUFA supplementation in oxidative stress in healthy subjects or in population with cardiometabolic disorders.

\begin{tabular}{|c|c|c|c|c|c|}
\hline Reference & Study Design & Population & Intervention & $\begin{array}{c}\text { Outcome } \\
\text { Measurements }\end{array}$ & Comments \\
\hline Meydani et al., 1991 [170] & $\begin{array}{c}\text { Randomized } \\
\text { intervention before } \\
\text { and after comparison }\end{array}$ & $\begin{array}{c}\text { Young females, aged } 51-71 ; n=14 . \text { Old } \\
\text { females, aged 51-71. } n=9\end{array}$ & $1680 \mathrm{mg}$ EPA $+720 \mathrm{mg}$ DHA per day for 3 months & Plasma MDA level & $\uparrow$ Plasma MDA level \\
\hline Nenseter et al., 1992 [176] & $\begin{array}{c}\text { Randomized } \\
\text { placebo-controlled } \\
\text { parallel clinical trial }\end{array}$ & $\begin{array}{c}\text { Normolipidemic subjects } \\
\text { Treatment: women and men, BMI not } \\
\text { reported, age: } 27-63, n=12 \\
\text { Control: women and men, BMI not reported, } \\
\text { age: } 23-70, n=11\end{array}$ & $\begin{array}{l}\text { Treatment: } 6 \mathrm{~g} \text { capsules/d of } n-3 \text { PUFA (highly } \\
\text { concentrated ethyl esters). } \\
\text { Control: } 6 \mathrm{~g} \text { of corn oil } \\
\text { Duration: } 4 \text { months }\end{array}$ & $\begin{array}{l}\text { Susceptibility of LDL } \\
\text { to Lipid peroxides } \\
\text { formation }\end{array}$ & $\begin{array}{l}\leftrightarrow \text { Lipid peroxides } \\
\text { formation }\end{array}$ \\
\hline Frankel et al., 1994 [177] & $\begin{array}{l}\text { Randomized, } \\
\text { double-blind, clinical } \\
\text { trial }\end{array}$ & $\begin{array}{l}\text { Hypertriglycemic men and women, age, BMI, } \\
\text { smoking status not reported. } n=9 / \text { group }\end{array}$ & $\begin{array}{l}\text { Control group: fish oil absent from the diet. } \\
\text { Supplemented group: } 5.1 \mathrm{~g} \text { of fish oil per day for } 6 \\
\text { weeks }\end{array}$ & $\begin{array}{l}\text { LDL oxidative } \\
\text { susceptibility }\end{array}$ & $\begin{array}{l}\leftrightarrow \text { LDL oxidative } \\
\text { susceptibility }\end{array}$ \\
\hline Brude et al., 1997 [178] & $\begin{array}{c}\text { Randomized, } \\
\text { double-blind, } \\
\text { placebo-controlled } \\
\text { parallel clinical trial }\end{array}$ & $\begin{array}{l}\text { Male smokers, hyperlipidemia, aged 40-60, } \\
\text { BMI not mentioned. } \\
n \text {-3 PUFA capsule group }(n=11) \text {, antioxidant } \\
\text { group }(n=11), n-3 \text { PUFAS }+ \text { antioxidants } \\
\text { group }(n=11) \text {, control oil group }(n=9)\end{array}$ & $\begin{array}{c}n \text {-3 PUFAS group: } 5 \mathrm{~g} \text { DHA and EPA/d } \\
\text { Antioxidants capsule, } 75 \mathrm{mg} \text { Vit E, } 150 \mathrm{mg} \text { Vit C, } 15 \\
\mathrm{mg} \beta \text {-carotene, and } 30 \mathrm{mg} \text { coenzyme Q10 per day } \\
\text { Control group: } 8 \mathrm{~g} \text { of oil with an FA pattern similar } \\
\text { to an ordinary Norwegian diet } \\
\text { Lasted for } 6 \text { weeks }\end{array}$ & $\begin{array}{l}\text { LDL oxidative } \\
\text { susceptibility, lipid } \\
\text { peroxides }\end{array}$ & $\begin{array}{l}\leftrightarrow \text { LDL oxidative } \\
\text { susceptibility, } \\
\leftrightarrow \text { lipid peroxides }\end{array}$ \\
\hline Mori et al., 1999 [159] & $\begin{array}{l}\text { Randomized, } \\
\text { controlled parallel } \\
\text { study }\end{array}$ & $\begin{array}{c}49 \text { untrained and sedentary NIDDM patients. } \\
\text { Age: } 30-65 \mathrm{y} . \\
\text { BMI }<36 \mathrm{~kg} / \mathrm{m}^{2}\end{array}$ & $\begin{array}{c}\text { Study 1: } \\
\text { Group 1: Low-fat diet (30\% of daily energy) }(n=14) \\
\text { Group 2: Low-fat diet }+ \text { one daily fish meal }(3.6 \mathrm{~g} \\
\quad n-3 \text { PUFA/day) }(n=12) \\
\text { Group 3: Low-fat diet + Moderate exercise }(n=11) \\
\text { Group 4: Low-fat diet + Fish meal + moderate } \\
\text { exercise }(n=12) \\
\text { for 8 weeks. }\end{array}$ & Urine F2- isoprostanes & Urine F2- isoprostanes \\
\hline Higdon et al., 2000 [160] & $\begin{array}{l}\text { Randomized blinded, } \\
\text { crossover study }\end{array}$ & $\begin{array}{l}\text { Post-menopausal women, aged between 50-75, } \\
\text { BMI }<30 \mathrm{~kg} / \mathrm{m}^{2} \text {, non-smokers, } n=15\end{array}$ & $\begin{array}{c}\text { Fish oil group: } 15 \mathrm{~g} / \mathrm{d}(2.0 \mathrm{~g} \mathrm{EPA} / \mathrm{d} \text { and } 1.4 \mathrm{~g} \text { DHA} / \mathrm{d}) \\
\text { Safflower oil group: } 15 \mathrm{~g} / \mathrm{d}(10.5 \mathrm{~g} \text { linoleate/ }) ; \\
\text { Sunflower oil: } 15 \mathrm{~g} / \mathrm{d}(12.3 \mathrm{~g} \text { oleate/d) in a } \\
\text { 3-treatment crossover trial }(5 \text { weeks with a } 7 \text {-wk } \\
\text { washout interval) }\end{array}$ & $\begin{array}{l}\text { Plasma } \\
\text { F2-isoprostanes, MDA, } \\
\text { and TBARS }\end{array}$ & $\begin{array}{l}\text { In fish oil group: } \\
\downarrow \text { plasma } \\
\text { F2-isoprostanes } \\
\downarrow \text { MDA } \\
\uparrow \text { TBARS }\end{array}$ \\
\hline
\end{tabular}


Table 2. Cont

\begin{tabular}{|c|c|c|c|c|c|}
\hline Reference & Study Design & Population & Intervention & $\begin{array}{c}\text { Outcome } \\
\text { Measurements }\end{array}$ & Comments \\
\hline Wander and Du, 2000 [172] & $\begin{array}{l}\text { Randomized } \\
\text { crossover study }\end{array}$ & $\begin{array}{l}\text { Post-menopausal women, aged } 45-75, \text { BMI < } \\
30 \mathrm{~kg} / \mathrm{m}^{2} \text {, smoking status not reported. } n=46\end{array}$ & $\begin{array}{c}\text { Group 1: fish oil (2.5 g EPA and } 1.8 \mathrm{~g} \text { DHA) } \\
\text { Group 2: fish oil (2.5 g EPA and } 1.8 \mathrm{~g} \text { DHA })+100 \\
\text { mg } \alpha \text {-tocopheryl acetate } \\
\text { Group 3: fish oil ( } 2.5 \mathrm{~g} \text { EPA and } 1.8 \mathrm{~g} \text { DHA })+200 \\
\text { mg } \alpha \text {-tocopheryl acetate } \\
\text { Group 4: fish oil (2.5 g EPA and } 1.8 \mathrm{~g} \text { DHA })+400 \\
\text { mg } \alpha \text {-tocopheryl acetate } \\
\text { for } 5 \text { weeks (4-period crossover design) }\end{array}$ & $\begin{array}{l}\text { TBARS, protein } \\
\text { oxidation }\end{array}$ & $\begin{array}{c}\uparrow \text { TBARS. Protein } \\
\text { oxidation not changed }\end{array}$ \\
\hline Mori et al., 2000 [162] & $\begin{array}{l}\text { Randomized, } \\
\text { placebo-controlled } \\
\text { parallel study }\end{array}$ & $\begin{array}{l}\text { Overweight, mildly hyperlipidemic men, age: } \\
20-65 \text { y, BMI: } 25-30 \mathrm{~kg} / \mathrm{m}^{2}\end{array}$ & $\begin{array}{l}\text { Group 1: } 4 \mathrm{~g} / \mathrm{d} \text { of purified EPA }(n=19) \\
\text { Group 2: } 4 \mathrm{~g} / \mathrm{d} \text { of purified DHA }(n=17) \\
\text { Group 3: } 4 \mathrm{~g} / \mathrm{d} \text { of olive oil }(n=20) \text { for } 6 \text { weeks }\end{array}$ & Urine F2- isoprostanes & $\begin{array}{l}\downarrow \text { Urine F2- } \\
\text { isoprostanes in the } \\
\text { EPA, DHA treatment } \\
\text { groups }\end{array}$ \\
\hline Wu et al., 2006 [179] & $\begin{array}{c}\text { Randomized, } \\
\text { single-blind, } \\
\text { placebo-controlled } \\
\text { parallel clinical trial } \\
\end{array}$ & $\begin{array}{c}\text { Post-menopausal vegetarian women, aged }<60 \text {. } \\
\text { Corn oil: } n=13 \\
\text { DHA: } n=14\end{array}$ & $\begin{array}{c}\text { Corn oil group: } 6 \mathrm{~g} \text { corn oil/day } \\
\text { DHA-rich algae oil group: } 2.14 \mathrm{~g} \text { of DHA/day } \\
\text { for } 6 \text { weeks }\end{array}$ & $\begin{array}{l}\text { Plasma } \alpha \text {-tocopherol, } \\
\text { urine F2-isoprostanes }\end{array}$ & $\begin{array}{l}\leftrightarrow \text { Plasma } \\
\alpha \text {-tocopherol, urine } \\
\text { F2-isoprostanes }\end{array}$ \\
\hline Egert et al., 2007 [173] & $\begin{array}{l}\text { Randomized parallel } \\
\text { controlled study }\end{array}$ & $\begin{array}{l}\text { Healthy men and women, aged: } 25.9 \pm 6.82 \\
\text { BMI: } 22.2 \pm 2.95 \text {, non-smokers. } n=48\end{array}$ & $\begin{array}{l}\text { ALA group: Rapeseed oil }+1 \% \text { of energy of ALA ( } n \\
=15) \\
\begin{array}{c}\text { EPA group: Rapeseed oil }+1 \% \text { of energy of EPA ( } n \\
=17)\end{array} \\
\begin{array}{r}\text { DHA group: Rapeseed oil }+1 \% \text { of energy of DHA } \\
(n=16)\end{array}\end{array}$ & $\begin{array}{l}\text { Ex vivo LDL oxidative } \\
\text { susceptibility }\end{array}$ & $\begin{array}{l}\text { EPA and DHA group: } \\
\uparrow \text { ex vivo LDL } \\
\text { oxidative } \\
\text { susceptibility }\end{array}$ \\
\hline Cazzola et al., 2007 [163] & $\begin{array}{l}\text { Randomized parallel } \\
\text { placebo-controlled } \\
\text { intervention }\end{array}$ & $\begin{array}{c}\text { Healthy young men (age: } 14-42 \text { y, BMI: } 24.1 \pm \\
0.3 \text { ). } n=93 \\
\text { Healthy old men (age: } 53-70 \text { y. BMI: } 27.6 \pm 0.0) \\
n=62\end{array}$ & $\begin{array}{c}4 \text { young and } 4 \text { older groups: } \\
\text { 1: } 1.35 \mathrm{~g} \mathrm{EPA}+0.27 \mathrm{~g} \text { DHA per day; } \\
\text { 2: } 2.7 \mathrm{~g} \text { EPA + 0.54 g DHA per day; } \\
\text { 3: } 4.05 \mathrm{~g} \text { EPA + } 0.81 \mathrm{~g} \text { DHA per day; } \\
\text { 4: corn oil group } \\
\text { Lasted for } 12 \text { weeks }\end{array}$ & $\begin{array}{l}\text { Plasma lipid } \\
\text { hydroperoxides } \\
\text { Lag time of } \\
\text { lipoprotein } \\
\text { peroxidation }\end{array}$ & $\begin{array}{c}\downarrow \text { Plasma lipid } \\
\text { hydroperoxides } \\
\downarrow \text { Lag time of } \\
\text { lipoprotein } \\
\text { peroxidation and } \\
\downarrow \text { GSH/Gluthatione in } \\
\text { olders }\end{array}$ \\
\hline Hanwell et al., 2009 [180] & $\begin{array}{c}\text { Randomized, } \\
\text { double-blind, } \\
\text { placebo-controlled } \\
\text { crossover clinical trial }\end{array}$ & $\begin{array}{l}\text { Hyper-triglyceridemic, overweight, and obese } \\
\text { men; aged }>45 \text {, smoking status not reported. } \\
\qquad n=10 \text { in total }\end{array}$ & $\begin{array}{c}\text { High-fat, high-fructose meal in all groups: } \\
\text { Fish oil group: } 7 \mathrm{~g} \text { of fish oil concentrate (2.8 g EPA } \\
\text { and } 1.4 \mathrm{~g} \text { DHA) } \\
\text { Isoflavone group: } 336 \mathrm{mg} \text { NovaSoy (150 mg } \\
\text { glycoside isoflavones). } \\
\text { Fish oil + isoflavone: } 7 \mathrm{~g} \text { fish oil + } 336 \mathrm{mg} \text { NovaSoy } \\
\text { Placebo group: } 7 \mathrm{~g} \text { corn oil } \\
\text { Consumed } 4 \text { days separated by 1week wash out. }\end{array}$ & $\begin{array}{l}\text { Lipid peroxides, } \\
\text { oxidized LDL, } \\
\text { total antioxidant } \\
\text { status }\end{array}$ & $\begin{array}{l}\leftrightarrow \text { Lipid peroxides, } \\
\leftrightarrow \text { oxidized LDL, } \\
\leftrightarrow \text { total antioxidant } \\
\quad \text { status }\end{array}$ \\
\hline
\end{tabular}


Table 2. Cont.

\begin{tabular}{|c|c|c|c|c|c|}
\hline Reference & Study Design & Population & Intervention & $\begin{array}{c}\text { Outcome } \\
\text { Measurements }\end{array}$ & Comments \\
\hline Bloomer et al., 2009 [174] & $\begin{array}{l}\text { Randomized, } \\
\text { double-blind } \\
\text { crossover study }\end{array}$ & $\begin{array}{c}\text { Subjects are exercise trained man, } \\
\text { non-smokers, no history of cardiometabolic } \\
\text { diseases. } \\
\text { Age: } 25.5 \pm 4.8 \text { y. BMI: } 24.1 \pm 1.6 \\
n=14\end{array}$ & $\begin{array}{l}\text { Intervention group: } 2.224 \mathrm{~g} \text { EPA and } 2.208 \mathrm{~g} \text { DHA } \\
\text { per day } \\
\text { Control group: same quantity of soybean oil } \\
\text { Duration: } 6 \text { weeks (with 8-week washout) } \\
\text { Supplementation were prior to performing a } 60 \text { min } \\
\text { treadmill climb using a weighted pack }\end{array}$ & $\begin{array}{l}\text { Blood was collected } \\
\text { pre and post exercise } \\
\text { and analyzed for a } \\
\text { variety of oxidative } \\
\text { stress (Protein } \\
\text { carbonyls, } \\
\text { IgG-autoantibodies, } \\
\text { low-density } \\
\text { lipoprotein, } \\
\text { Malondialdehyde, } \\
\text { Hydrogen peroxide } \\
\text { and xanthine oxidase } \\
\text { activity, Nitric oxide, } \\
\text { Whole blood lactate } \\
\text { and inflammatory } \\
\text { biomarkers }\end{array}$ & $\begin{array}{c}\text { Resting levels: } \\
\downarrow \text { CRP, } \downarrow \text { TNF- } \alpha, \leftrightarrow \\
\text { MDA, } \\
\leftrightarrow \text { Nitric oxide. } \\
\text { Exercise: } \\
\uparrow \text { oxidative } \\
\text { biomarkers (mild) }\end{array}$ \\
\hline Mas et al., 2010 [164] & $\begin{array}{l}\text { Randomized, } \\
\text { Placebo-controlled } \\
\text { intervention }\end{array}$ & $\begin{array}{l}\text { Study A: placebo-controlled intervention (BMI: } \\
\text { 25-30), dyslipidemic men, age: } 20-54 \mathrm{y}, n= \\
17-20 \text { per group. } \\
\text { Study B: hypertensive type } 2 \text { diabetic and } \\
\text { post-menopausal women, age: } 40-75 \mathrm{y}, n= \\
16-18\end{array}$ & $\begin{array}{c}\text { In both studies, } \\
\text { n-3 PUFA group: } 4 \text { g/day of EPA or DHA } \\
\text { Control group: Olive oil placebo } \\
\text { lasted for } 6 \text { weeks }\end{array}$ & $\begin{array}{c}\text { Plasma } \\
\text { F2-isoprostanes }\end{array}$ & $\begin{array}{c}\downarrow \text { Plasma } \\
\text { F2-isoprostanes with } \\
n \text {-3 PUFAS } \\
\text { supplementation }\end{array}$ \\
\hline Ulven et al., 2011 [182] & $\begin{array}{l}\text { Randomized parallel } \\
\text { study }\end{array}$ & $\begin{array}{l}\text { Participants with normal or slightly elevated } \\
\text { total blood cholesterol and/or triglyceride } \\
\text { levels, age: } 30-50 \mathrm{y}, \mathrm{BMI}>30 \mathrm{~kg} / \mathrm{m}^{2}\end{array}$ & $\begin{array}{c}\text { Krill oil group: } 3 \mathrm{~g} / \text { day }(\mathrm{EPA}+\mathrm{DHA}=543 \mathrm{mg} / \text { day }) \\
\text { in } 6 \text { capsules }(n=36) \\
\text { Fish oil group: } 1.8 \mathrm{~g} / \text { day }(\mathrm{EPA}+\mathrm{DHA}=864 \mathrm{mg} / \text { day }) \\
\text { in } 3 \text { capsules }(n=40) \\
\text { Control group: no supplementation }(n=37) \\
\text { Duration: } 7 \text { weeks }\end{array}$ & $\begin{array}{l}\text { Urine F2-isoprostanes, } \\
\text { plasma } \alpha \text {-tocopherol }\end{array}$ & $\begin{array}{l}\leftrightarrow \text { Urine } \\
\text { F2-isoprostanes, } \\
\leftrightarrow \text { plasma } \\
\alpha \text {-tocopherol }\end{array}$ \\
\hline Egert et al., 2012 [184] & $\begin{array}{l}\text { Randomized } \\
\text { single-blind parallel }\end{array}$ & $\begin{array}{c}\text { Men and premenopausal women; Age: } 19-43 \\
\text { y; BMI }<28 \mathrm{~kg} / \mathrm{m}^{2} \text {, non-smokers }\end{array}$ & $\begin{array}{c}\text { Margarines fortified with } 10 \% \text { weight of EPA, DHA, } \\
\text { or ALA } \\
\text { EPA group: } 2.2 \mathrm{~g} / \text { day }(n=25) \\
\text { DHA group: } 2.3 \mathrm{~g} / \text { day }(n=25) \\
\text { ALA group: } 4.4 \mathrm{~g} / \text { day }(n=24) \\
\text { For } 6 \text { weeks }\end{array}$ & $\begin{array}{l}\text { Antioxidant capacity, } \\
\text { plasma MDA, } \\
\text { RBC-MDA, linoleic } \\
\text { acid hydroperoxides } \\
\text { (LA-OOH) in RBC }\end{array}$ & $\begin{array}{c}\leftrightarrow \text { Antioxidant } \\
\text { capacity } \\
\uparrow \text { Plasma MDA in EPA } \\
\text { and DHA groups. } \\
\leftrightarrow \text { RBC-MDA } \\
\downarrow \text { RBC-LA-OOH }\end{array}$ \\
\hline
\end{tabular}


Table 2. Cont.

\begin{tabular}{|c|c|c|c|c|c|}
\hline Reference & Study Design & Population & Intervention & $\begin{array}{c}\text { Outcome } \\
\text { Measurements }\end{array}$ & Comments \\
\hline Kirkhus et al., 2012 [185] & $\begin{array}{l}\text { Open, randomized } \\
\text { parallel study }\end{array}$ & $\begin{array}{l}159 \text { healthy men and women. Age: } 18-70 \mathrm{y} \text {, } \\
\mathrm{BMI}<30 \mathrm{~kg} / \mathrm{m}^{2} \text {, moderate smokers }\end{array}$ & $\begin{array}{c}\text { 1g/day of EPA + DHA as: } \\
\text { - fish pâté ( } 34 \mathrm{~g}) \cdot n=44 \\
\text { - n-3 PUFA-enriched fruit juice }(500 \mathrm{~mL}) . n=38 \\
-3 \text { capsuled of fish oil. } n=40 \\
\text { - Control: non-supplemented. } n=37 \\
\text { Duration: 7weeks }\end{array}$ & $\begin{array}{l}\text { Urine F2-isoprostanes } \\
\text { and plasma } \\
\alpha \text {-tocopherol }\end{array}$ & $\begin{array}{l}\downarrow \text { Plasma } \alpha \text {-tocopherol } \\
\text { in fish pâté group } \\
\text { when calculated in } \\
\text { relation to the level of } \\
\text { serum TG } \\
\leftrightarrow \text { F2-isoprostanes }\end{array}$ \\
\hline Ottestad et al., 2012 [183] & $\begin{array}{l}\text { Randomized, } \\
\text { double-blind, } \\
\text { placebo-controlled } \\
\text { parallel study }\end{array}$ & $\begin{array}{l}54 \text { Healthy men and women, age: } 18-50 \mathrm{y} \text {, BMI } \\
<30 \mathrm{~kg} / \mathrm{m}^{2} \text {, non-smokers }\end{array}$ & $\begin{array}{c}\text { Group 1: } 8 \mathrm{~g} / \mathrm{d} \text { of fish oil (EPA/DHA) } n=17 \\
\text { Group 2: } 8 \mathrm{~g} / \mathrm{d} \text { of oxidized fish oil (EPA/DHA) } n=18 \\
\text { Group 3: } 8 \mathrm{~g} / \mathrm{d} \text { of high-oleic sunflower oil } n=19 \\
\text { For } 7 \text { weeks }\end{array}$ & $\begin{array}{l}\text { Urine F2-isoprostanes } \\
\text { and plasma oxidation } \\
\text { products from n-3 } \\
\text { PUFA and n-6 PUFA } \\
\text { oxidation 4-HHE and } \\
\text { 4-HNE; plasma } \\
\alpha \text {-tocopherol, } \\
\text { enzymatic activity of } \\
\text { GR, GPx, and CAT }\end{array}$ & $\begin{array}{c}\leftrightarrow \text { Urine } \\
\text { F2-isoprostanes and } \\
\leftrightarrow \text { plasma oxidation } \\
\text { products from n-3 } \\
\text { PUFA and n-6 PUFA } \\
\text { oxidation } \\
\leftrightarrow \text { 4-HHE and } \leftrightarrow \\
\text { 4-HNE; } \\
\leftrightarrow \text { plasma } \\
\alpha \text {-tocopherol, } \\
\leftrightarrow \text { enzymatic activity } \\
\text { of GR, GPx, and CAT }\end{array}$ \\
\hline Schimidt et al., 2012 [165] & $\begin{array}{l}\text { Randomized, } \\
\text { controlled, parallel } \\
\text { intervention }\end{array}$ & $\begin{array}{l}10 \text { normo and } 10 \text { dyslipidemic men; Age: } \\
29-51, \text { BMI: } 35 \mathrm{~kg} / \mathrm{m}^{2} . n=20\end{array}$ & $\begin{array}{l}6 \text { Fish oil capsules, providing } 1.14 \mathrm{~g} \text { DHA and } 1.56 \mathrm{~g} \\
\text { EPA per day, for } 12 \text { weeks }\end{array}$ & $\begin{array}{c}\text { GST, GR, and } \\
\text { antioxidative enzymes } \\
\text { SOD3, CAT, and } \\
\text { HMOX2 expression in } \\
\text { whole blood cells, } \\
\text { GPx, MMPs, } \\
\text { cyrochrome P450 } \\
\text { (CYP) enzymes } \\
\text { expression in whole } \\
\text { blood }\end{array}$ & $\begin{array}{c}\uparrow G S T, \uparrow G R \text { and } \\
\text { antioxidative enzymes } \\
\uparrow \text { SOD3, } \uparrow \text { CAT and } \\
\text { HMOX2 expression, } \\
\downarrow \text { GPx, } \\
\downarrow \text { MMPs, } \downarrow \text { cytochrome } \\
\text { P450 (CYP) enzymes } \\
\text { expression }\end{array}$ \\
\hline $\begin{array}{l}\text { Kiecolt-Glaser et al., } 2013 \\
\text { [166] }\end{array}$ & $\begin{array}{l}\text { Randomized, } \\
\text { double-blind, } \\
\text { controlled parallel } \\
\text { trial }\end{array}$ & $\begin{array}{c}\text { Healthy sedentary overweight middle-aged } \\
\text { and older adults } \\
\text { Age: } 48-85 \text { y, BMI: } 22.5-40 \mathrm{~kg} / \mathrm{m}^{2} . \\
\text { Non-smokers }\end{array}$ & $\begin{array}{c}\text { Group 1: } 2.5 \mathrm{~g} / \text { day } \mathrm{n}-3 \text { PUFA }(n=35), \\
\text { Group 2: } 1.25 \mathrm{~g} / \text { day } \mathrm{n}-3 \text { PUFA }(n=40) \\
\text { Group 3: placebo capsules that mirrored the } \\
\text { proportions of fatty acids in the typical American } \\
\text { diet }(n=31) \\
\text { Duration: } 4 \text { months }\end{array}$ & $\begin{array}{c}\text { Plasma } \\
\text { F2-isoprostanes }\end{array}$ & $\begin{array}{l}\downarrow \text { Plasma } \\
\text { F2-isoprostanes with } \\
\text { n-3 PUFAS } \\
\text { supplementation }\end{array}$ \\
\hline Haijianfar et al., 2013 [167] & $\begin{array}{l}\text { Randomized } \\
\text { double-blind } \\
\text { placebo-controlled } \\
\text { clinical trial }\end{array}$ & $\begin{array}{l}\text { Type } 2 \text { diabetic women. Age: } 45-65 \text { y } \\
\text { BMI: } 27.7 \pm 3.4 \text { (n-3 PUFA group). } \\
\text { BMI: } 28 \pm 3.8 \text {. (Control group) }\end{array}$ & $\begin{array}{l}\text { n-3 PUFA group: } 2000 \mathrm{mg} / \mathrm{d} \text { in } 2 \text { capsules: each } \\
\text { contained 1,000 mg n-3PUFA (65\% EPA, 360 mg and } \\
35 \% \text { DHA, } 240 \mathrm{mg})(n=37) \\
\text { Control group: } 2 \text { placebo capsules, each contains } 1 \mathrm{~g} \\
\text { of cornstarch }(n=34) \\
\text { Duration: } 8 \text { weeks }\end{array}$ & $\begin{array}{l}\text { Serum antioxidant } \\
\text { capacity }\end{array}$ & $\begin{array}{l}\uparrow \text { Antioxidant capacity } \\
\text { in the n-3 PUFA } \\
\text { supplemented group }\end{array}$ \\
\hline
\end{tabular}


Table 2. Cont.

\begin{tabular}{|c|c|c|c|c|c|}
\hline Reference & Study Design & Population & Intervention & $\begin{array}{c}\text { Outcome } \\
\text { Measurements }\end{array}$ & Comments \\
\hline Véricel et al. 2015 [168] & $\begin{array}{c}\text { Randomized, } \\
\text { double-blind, } \\
\text { placebo-controlled, } \\
\text { two-period crossover } \\
\text { trial }\end{array}$ & $\begin{array}{l}\text { Post-menopausal women with type } 2 \text { diabetes, } \\
\text { age: } 59.8 \pm 4.7 \mathrm{y} \text {, BMI: } 34.1 \pm 5 \mathrm{~kg} / \mathrm{m}^{2} . n=11\end{array}$ & $\begin{array}{c}\text { Intervention: } 400 \mathrm{mg} / \mathrm{day} \text { of DHA (in } 2 \text { capsules/d) } \\
\text { Control: } 2 \text { placebo (same amount of sunflower oil) } \\
\text { Duration: } 2 \text { weeks }\end{array}$ & $\begin{array}{l}\text { Plasma and platelet } \\
\text { vitamin E, alpha- and } \\
\text { gamma-tocopherol } \\
\text { concentrations, } \\
\text { plasma MDA, } \\
\text { 8-iso-PGF2 } \alpha\end{array}$ & $\begin{array}{c}\uparrow \text { Platelet } \\
\text { alpha-tocopherol, } \\
\text { gamma-tocopherol } \\
\text { tend to increase. } \\
\downarrow \text { MDA, } \\
\downarrow 8 \text {-iso-PGF2 } \alpha \text {. } \\
\text { n-3 PUFAS } \\
\text { supplementation } \\
\downarrow \text { oxidative stress } \\
\text { associated with } \\
\text { diabetes }\end{array}$ \\
\hline $\begin{array}{l}\text { Alves Luzia et al. (2015) } \\
\text { [175] }\end{array}$ & $\begin{array}{l}\text { Randomized, } \\
\text { double-blind, } \\
\text { placebo-controlled } \\
\text { trial }\end{array}$ & $\begin{array}{l}\text { Women ( } 40 \text { to } 70 \text { years) with low habitual fatty } \\
\text { fish and seafood intake, who met at least two } \\
\text { of the following criteria: total cholesterol }>200 \\
\mathrm{mg} / \mathrm{dL} \text {, LDL-C }>140 \mathrm{mg} / \mathrm{dL} \text {, HDL-C }<50 \\
\mathrm{mg} / \mathrm{dL} \text {, and triglycerides }>150 \mathrm{mg} / \mathrm{dL}\end{array}$ & $\begin{array}{c}\text { The fish oil group: daily consumption of } 1 \mathrm{~g} \mathrm{n}-3 \\
\text { PUFA (540 mg EPA + } 360 \mathrm{mg} \text { DHA) and } 1 \text { capsule of } \\
\text { placebo }(n=22) \\
\text { Fish+VitE group: } 1 \mathrm{~g} \text { n-3 PUFA, } 400 \mathrm{mg} \text { vitamin E/ } \\
\text { alpha-tocopherol }(n=19) \text {. } \\
\text { Placebo group: } 2 \text { capsules/d mineral oil }(n=18) \\
\text { Duration: } 3 \text { months }\end{array}$ & $\begin{array}{c}\text { Biomarkers of } \\
\text { oxidative stress at } \\
\text { baseline, } 45 \text { and } 90 \\
\text { days }\end{array}$ & $\begin{array}{l}\uparrow \text { TBARS in the group } \\
\text { supplemented with } \\
\text { fish oil alone, but not } \\
\text { in the fish oil }+ \\
\text { vitamin E group }\end{array}$ \\
\hline Berge et al., (2015) [169] & $\begin{array}{l}\text { Randomized, clinical } \\
\text { interventional pilot } \\
\text { study }\end{array}$ & $\begin{array}{l}\text { Healthy female and male, mean age: } 23 \pm 4 \mathrm{y} \\
\text { BMI: } 20.9 \mathrm{~kg} / \mathrm{m}^{2}, \mathrm{n}=17\end{array}$ & $\begin{array}{c}17 \text { subjects received dietary supplementation with } \\
\text { krill oil ( } 832.5 \mathrm{mg} \text { EPA and DHA per day) for } 28 \\
\text { days }\end{array}$ & $\begin{array}{l}\text { Plasma total } \\
\text { antioxidant capacity } \\
\text { (AOC) }\end{array}$ & $\begin{array}{l}\uparrow A O C \text { after krill oil } \\
\text { intake. AOC } \\
\text { positively correlated } \\
\text { with plasma EPA } \\
\text { concentration and } \\
\text { RBC EPA } \\
\text { concentration } \\
\end{array}$ \\
\hline Fayh et al., 2018 [161] & $\begin{array}{l}\text { Randomized, } \\
\text { double-blind, } \\
\text { placebo-controlled } \\
\text { trial }\end{array}$ & $\begin{array}{l}\text { Male and female with T2DM, Age: } 50-57 \mathrm{y} \text {. } \\
\text { Mean BMI: } 28.2 \mathrm{~kg} / \mathrm{m}^{2} \text { in n-3 PUFAS group } \\
\text { and } 28.8 \mathrm{~kg} / \mathrm{m}^{2} \text { in control group. } n=15 / \text { group }\end{array}$ & $\begin{array}{c}\text { Control group: } 3 \text { capsules/day that contains } 500 \mathrm{mg} \\
\text { gelatin } \\
\text { Intervention group: } 3 \text { capsules/d (each capsule } \\
\text { contains } 180 \mathrm{mg} \text { EPA, } 120 \mathrm{mg} \text { DHA, } 2 \mathrm{mg} \text { Vit E) } \\
\text { For } 8 \text { weeks } \\
\text { At the beginning and at the end of protocol, an acute } \\
\text { exercise was performed (treadmill) }\end{array}$ & $\begin{array}{l}\text { TBARS; Plasma } \\
\text { F2-isoprostanes, TRAP, } \\
\text { SOD activity, hs-CRP }\end{array}$ & $\begin{array}{c}\text { n-3 PUFA } \\
\text { supplementation: } \\
\downarrow \text { TG, } \downarrow \text { TRAP levels } \\
\text { after exercise, without } \\
\text { a significant effect on } \\
\text { inflammatory and } \\
\text { oxidative-stress } \\
\text { markers }\end{array}$ \\
\hline
\end{tabular}

Abbreviations: NAFLD, non-alcoholic fatty liver disease; AST, aspartate transaminase; ALT, alanine transaminase; GGT, gamma-glutamyl transpeptidase; $\uparrow$, increased; $\downarrow$, decreased; $\leftrightarrow$, not changed; TG, triglycerides; US, ultrasonography; DPI, doppler perfusion index; TNF-alpha, tumor necrosis factor-alpha; HOMA-IR, homeostasis model assessment-estimated IR; HDL, high density lipoprotein; FBG, fasting blood glucose; NS, not significant; CRP, C-reactive protein; MDA, malondialdehyde; EPA-E, ethyl-eicosapentanoic acid; MRI, magnetic resonance imaging; NAS, NASH activity score; ApoB, apolipoprotein B, FGF21, fibroblast growth factor 21; PGE2, prostaglandin E2; Hb1C, Hemoglobin A1c. 
In addition to quantifying the oxidative stress produced by free radicals, several molecules including lipids, DNA and proteins were considered as the main biomarkers since they could be modified by excessive ROS in the microenvironment [186]. Most of the 27 studies above measured the lipid oxidation and its products since they were considered as the widely used biomarker of oxidative stress.

F2-isoprostane is a chemically stable prostaglandin-like isomer generated by the reaction of polyunsaturated fatty acids in membrane phospholipids and free radicals or ROS [187-190]. F2-isoprostane has been considered as a gold standard lipid peroxidation marker and was identified as an excellent and sensitive biomarker of in vivo lipid peroxidative damage. Indeed, F2-isoprostanes have been found to be elevated in syndromes putatively associated with oxidative stress and aging such as type 2 diabetes, Alzheimer's disease and NAFLD [13-15]. After n-3 PUFA supplementation, several clinical trials have reported decreased urine and/or plasma F2-isoprostane levels in humans $[159,160,162,164,166,168]$. However, other trials have found no effects on F2-isoprostanes [179,181-183,185]. Most of the trials have been performed using n-3 PUFA preparations that contain both EPA and DHA. A few trials performed comparative studies on the effects of EPA and DHA themselves. In this context, Mori et al. [162] demonstrated that both purified EPA and DHA equally reduced urine F2-isoprostanes, suggesting that at least in the short term, the inclusion of regular fish meals providing n-3 PUFA or the supplementation with purified EPA and DHA, can reduce in vivo oxidative stress in humans. Similar outcomes were found in a trial with overweight subjects, mildly hyperlipidemic men, treated with purified EPA or DHA for 6 weeks [164].

Oxidized LDL (oxLDL) levels have been also used as biomarkers of oxidative stress since, low-density lipoproteins can undergo oxidative modification. Regarding the effects of n-3 PUFA supplementation on the oxLDL as an oxidative-stress biomarker, some of them found no significant changes [176-178,180], while others revealed that oxLDL susceptibility increased [171,173]. It should be mentioned that the use of oxidized LDL as a biomarker of oxidative stress has been criticized and that the inconsistency of the results obtained in the different trials may be due to the heterogeneity of oxidation products, the low specificity of the antibodies and the different results obtained depending on the assay used [191-193].

Malondialdehyde (MDA) is an end-product of lipid peroxidation of polyunsaturated fatty acids including AA. Circulating MDA is one of the most commonly and widely used biomarkers of oxidative stress [194]. As reported for other oxidative-stress biomarkers, the role of n-3 PUFA supplementation on MDA plasma levels remains unclear. Thus, some trials observed that after n-3 PUFA supplementation, MDA plasma levels decreased $[160,168]$. Other studies demonstrated increased levels of MDA in plasma $[170,172,173,175,184]$. Various analytical methods have been used to measure MDA in biological samples. The most common is the Thiobarbituric acid reactive substances (TBARS) method [195]. In a study conducted by Higdon et al. [160], MDA level decreased while TBARS level increased. In this regard, TBARS lacks specificity, as many chemically reactive carbonyl groups-containing compounds from different classes of substances including oxidized polyunsaturated fatty acids and carbohydrates, from endogenous sources and foods present in body fluids, can react with TBA. Therefore, it should be taken into consideration that results might vary between studies carried out by TBARS or other more specific techniques for the measurement of MDA [195].

At the clinical level, other biomarkers of oxidative stress analyzed were those related to antioxidant defenses such as CAT, GR, GPx and Heme oxygenase 2 (HMOX2) enzymes [123], plasma GSH and $\alpha$-tocopherol levels, and total antioxidant capacity $[167,179,182,183]$. A few studies evaluated the effects of n-3 PUFA on these biomarkers and although some of these showed improved antioxidant defenses levels or total antioxidant capacity $[165,167,169]$, others described neutral effects $[179,180,182,183]$.

The mechanism by which oxidative stress could be reduced following n-3 PUFA supplementation is still unresolved, but it has been assumed that these effects may occur through immunomodulation and a decreased leukocyte activation [196]. In this sense, it is known that activated immune cells produce cytokines (i.e., TNF- $\alpha$ or IL-6) that consequently promote ROS generation $[174,197,198]$. Interestingly, 
numerous studies have demonstrated the ability of n-3 PUFA to diminish pro-inflammatory cytokine production [108]. Moreover, it has been proposed that EPA and DHA are more effective acting as antioxidants and as superoxide scavengers in an unsaturation-dependent manner, given the high unsaturation level of n-3 PUFA [109]. Additionally, EPA and DHA can replace AA in cell membranes decreasing AA concentration [199], which serves as a precursor of F2-isoprostanes.

As a matter of fact, it remains controversial whether n-3 PUFA are effective to counteract oxidative stress in humans. Indeed, the heterogeneity in population, treatment duration, doses, and methods to assess oxidative stress in the trials reviewed prevent any definitive conclusion.

\section{3. n-3 PUFA Supplementation in NAFLD and NASH Adult Patients}

To evaluate the potential beneficial effect of supplementing n-3 PUFA (including DHA and EPA) in NAFLD and NASH, another systematic review of clinical trials carried out in adults was performed. Potentially relevant studies were retrieved by a systematic search in the PubMed database. The following search terms were used: "n-3 PUFA and NAFLD", "DHA and NAFLD", "EPA and NAFLD", "n-3 PUFA and NASH", "DHA and NASH", "EPA and NASH". Articles were filtered by clinical trials (article type), full text (availability), 10 years (publication date), and only human trials were included. Studies which recruited obese adults ( $>18$ years old) with pre-diagnosed NAFLD at different stages and with liver or blood profile inflammation and oxidative stress related biomarkers were included, as well as studies in patients with NAFLD or NASH associated with diabetes and hyperlipidemia. Studies including a specific ethnic population, NAFLD or NASH associated with cardiovascular diseases or risk factors, polycystic ovary syndrome, and genotypes among others were excluded. Around 1501 non-duplicated entries, with the applied filters of "clinical trials" and "human" were found in the PubMed database. Titles and abstracts were reviewed for the first selection, and full texts were checked only when abstracts themselves were not able to reveal the nature of the studies. Finally, 13 clinical trials were included (Figure 3).

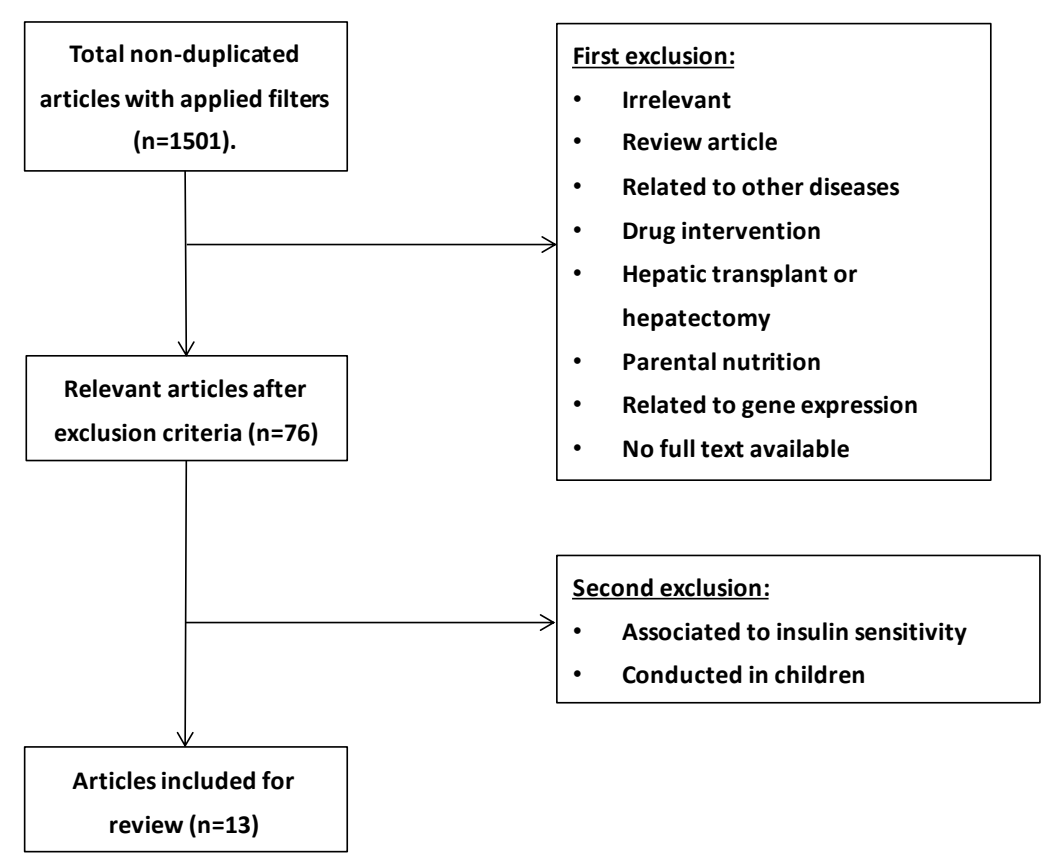

Figure 3. Flowchart of selection process based on n-3 PUFA and NAFLD/NASH.

Table 3 describes the characteristics (population, design, etc.) of the selected trials as well as the main findings concerning the effects of n-3 PUFA supplementation in adult patients with NAFLD and NASH. 
Table 3. Effects of n-3 PUFA supplementation in NAFLD (non-alcoholic fatty liver disease) and NASH (non-alcoholic steatohepatitis) adults.

\begin{tabular}{|c|c|c|c|c|c|c|}
\hline Reference & Study Design & Population & Intervention & $\begin{array}{c}\text { Outcome } \\
\text { Measurements }\end{array}$ & Results & Comments \\
\hline $\begin{array}{l}\text { Capanni et al., } \\
\text { 2006. [200] }\end{array}$ & Open-label trial & $\begin{array}{c}\text { Patients with NAFLD } \\
\text { proven by US; Age range: } \\
\text { 31-77 y. Mean BMI: } 28.5 \\
\mathrm{~kg} / \mathrm{m}^{2} . n=56\end{array}$ & $\begin{array}{l}\text { Oral intake of n-3 PUFA (EPA } \\
\text { and DHA in a } 0.9 / 1.5 \text { ratio), } 1 \\
\text { g capsule a day for } 12 \text { months. } \\
\text { Intervention group ( } n=42) \\
v s \text { control group }(n=14)\end{array}$ & $\begin{array}{l}\text { Hematochemical tests; } \\
\text { Liver fat changes } \\
\text { detected by US and } \\
\text { liver eco-texture } \\
\text { measured by Duplex } \\
\text { Doppler US and DPI } \\
\text { follow up }\end{array}$ & $\begin{array}{c}\downarrow \text { AST, ALT, GGT; } \\
\downarrow \text { fasting TG and glucose } \\
\downarrow \text { arachidonate } \\
\downarrow \text { n- } 6 / \text { n- } 3 \text { ratio } \\
\text { Significant beneficial effects } \\
\text { on liver US pattern and } \uparrow \text { DPI }\end{array}$ & $\begin{array}{l}\text { Long-term n-3 PUFAS } \\
\text { supplementation } \\
\text { ameliorates hepatic } \\
\text { steatosis in NAFLD } \\
\text { patients }\end{array}$ \\
\hline $\begin{array}{l}\text { Spadaro et al., } \\
\text { 2008. [111] }\end{array}$ & $\begin{array}{l}\text { Randomized } \\
\text { open-label trial }\end{array}$ & $\begin{array}{c}\text { Patients with NAFLD } \\
\text { proven by US; Mean age: } \\
51 \text { y Mean BMI: } 30.5 \\
\text { kg/m² }\end{array}$ & $\begin{array}{l}\text { AHA diet }+2 \mathrm{~g} / \mathrm{d} \text { n-3 PUFA } \\
(\text { group DP, } n=20) \\
\text { AHA diet }(\text { Group D, } n=20) \\
\text { for } 6 \text { months }\end{array}$ & $\begin{array}{l}\text { Changes on liver fat } \\
\text { via US; ALT, AST, } \\
\text { GGT, lipid profile, } \\
\text { TNF- } \alpha \text { serum levels, } \\
\text { fasting glucose, and } \\
\text { IR by HOMA-IR }\end{array}$ & $\begin{array}{c}\text { Group DP: } \\
\downarrow \text { ALT, GGT } \\
\downarrow \text { TG, TNF- } \alpha \\
\downarrow \text { HOMA-IR } \\
\uparrow \text { HDL cholesterol } \\
\text { Complete steatosis regression } \\
\text { in } 33.4 \% \text { of patients and an } \\
\text { overall reduction of } 50 \% .\end{array}$ & $\begin{array}{l}\text { n-3 PUFA have a major } \\
\text { improvement on fatty liver } \\
\text { in patients with NAFLD }\end{array}$ \\
\hline $\begin{array}{l}\text { Zhu et al., } \\
\text { 2008. [201] }\end{array}$ & $\begin{array}{l}\text { Randomized } \\
\text { controlled trial }\end{array}$ & $\begin{array}{c}\text { Patients with US proven } \\
\text { NAFLD associated with } \\
\text { hyperlipidemia; Age: } \\
18-65 \mathrm{y}\end{array}$ & $\begin{array}{l}\text { Oral supplementation of n-3 } \\
\text { PUFA for } 24 \text { weeks. } \\
\text { AHA-based diet with a } \\
\text { caloric restriction of } 25-30 \\
\text { kcal/kg per day } \\
\text { Group A }(n=66): 2 \mathrm{~g} \mathrm{n}-3 \\
\text { PUFA from seal oils, } 3 \\
\text { times/day. Group B }(n=68) 2 \\
\text { g placebo, three times/day }\end{array}$ & $\begin{array}{c}\text { Primary endpoints: } \\
\text { fatty liver assessed by } \\
\text { symptom scores, ALT } \\
\text { and serum lipid levels } \\
\text { at } 8,12,16 \text {, and } 24 \\
\text { weeks. } \\
\text { Secondary endpoints: } \\
\text { liver fat changes by } \\
\text { US at weeks } 12 \text { and } 24\end{array}$ & $\begin{array}{c}\text { After } 24 \text { wk of treatment: } \\
\leftrightarrow \text { body weight, } \leftrightarrow \text { FBG; } \\
\downarrow \text { Total symptom scores, } \\
\downarrow \text { ALT and TG } \\
\text { Complete fatty liver } \\
\text { regression was observed in } \\
19.7 \% \text { of the patients, and an } \\
\text { overall reduction was found } \\
\text { in } 53.0 \% \text { (35/66) of the } \\
\text { patients in group A }\end{array}$ & $\begin{array}{l}\text { n-3 PUFA from seal oils is } \\
\text { safe and efficacious for } \\
\text { patients with NAFLD } \\
\text { associated with } \\
\text { hyperlipidemia and can } \\
\text { improve their total } \\
\text { symptom scores, ALT, } \\
\text { serum lipid levels, and } \\
\text { normalization of } \\
\text { ultrasonographic evidence }\end{array}$ \\
\hline $\begin{array}{l}\text { Tanaka et al., } \\
\text { 2008. [202] }\end{array}$ & Pilot Trial & $\begin{array}{l}23 \text { biopsy-proven } \\
\text { NASH patients }\end{array}$ & $\begin{array}{l}\text { Highly purified EPA ( } 2700 \\
\text { mg/d) was administered for } \\
12 \text { months }\end{array}$ & $\begin{array}{l}\text { Biochemical } \\
\text { parameters of glucose } \\
\text { and lipid metabolism, } \\
\text { inflammatory and } \\
\text { iron metabolism } \\
\text { oxidative-stress } \\
\text { markers } \\
\text { Ultrasonography } \\
\text { Histologic evaluation } \\
\text { of liver biopsies }\end{array}$ & $\begin{array}{c}\downarrow \text { ALT, AST } \\
\downarrow \text { Total cholesterol } \\
\downarrow \text { sTNFR1,2 } \\
\downarrow \text { Ferritin } \\
\downarrow \text { Thioredoxin } \\
\downarrow \text { hepatic steatosis and } \\
\text { fibrosis, hepatocyte } \\
\text { ballooning, and } \\
\text { lobular inflammation }\end{array}$ & $\begin{array}{l}\text { EPA treatment seems to be } \\
\text { safe and efficacious for } \\
\text { patients with } \\
\text { NASH }\end{array}$ \\
\hline
\end{tabular}


Table 3. Cont

\begin{tabular}{|c|c|c|c|c|c|c|}
\hline Reference & Study Design & Population & Intervention & $\begin{array}{c}\text { Outcome } \\
\text { Measurements }\end{array}$ & Results & Comments \\
\hline $\begin{array}{l}\text { Sofi et al., } \\
\text { 2010. [203] }\end{array}$ & Randomized & $\begin{array}{l}\text { Patients with NAFLD } \\
\text { proven by US. Age: } 30-70 \\
\text { y, mean BMI: } 29.3 \mathrm{~kg} / \mathrm{m}^{2}\end{array}$ & $\begin{array}{c}\text { Food consumption enriched } \\
\text { with n-3 PUFA }(0.47 \mathrm{~g} \text { EPA + } \\
0.24 \mathrm{~g} \text { DHA) for } 12 \mathrm{months} \\
\text { Group 1: }(n=6) 6.5 \mathrm{ml} / \mathrm{d} \\
\text { enriched with olive oil + } \\
\text { recommended diet } \\
\text { Group 2: }(n=5) \text { control } \\
\text { (recommended diet }+ \text { not } \\
\text { enriched olive oil) }\end{array}$ & $\begin{array}{l}\text { Liver eco-texture } \\
\text { measured by Duplex } \\
\text { Doppler US and DPI. } \\
\text { Liver enzymes, TG } \\
\text { and adiponectin } \\
\text { levels }\end{array}$ & $\begin{array}{c}\downarrow \text { ALT, AST, and GGT } \\
\downarrow \text { TG level } \\
\uparrow H D L \text { cholesterol } \\
\uparrow \text { adiponectin } \\
\uparrow \text { DPI level }\end{array}$ & $\begin{array}{l}\text { Persistent consumption of } \\
\text { food enriched with n-3 } \\
\text { PUFA has favorable effects } \\
\text { in patients with NAFLD }\end{array}$ \\
\hline $\begin{array}{l}\text { Scorletti et al., } \\
\text { 2014. [204] }\end{array}$ & $\begin{array}{l}\text { WELCOME study: } \\
\text { double-blind, } \\
\text { randomized, } \\
\text { placebo-controlled } \\
\text { trial }\end{array}$ & $\begin{array}{l}\text { Patients with histological } \\
\text { confirmation of NAFLD. } \\
\text { Mean age: } 50 \text { years old. } \\
\text { Mean BMI: } 32.5 \mathrm{~kg} / \mathrm{m}^{2}\end{array}$ & $\begin{array}{l}\text { Intervention group }(n=51) \text { : } \\
\text { oral supplementation of } \\
\text { purified long-chain } n-3 \text { PUFA } \\
\text { ethyl esters ( } 1 \text { g contains } 460 \\
\text { mg of EPA and } 380 \text { mg of } \\
\text { DHA), } 4 \mathrm{~g} / \text { day. } \\
\text { Placebo group }(n=52): \\
4 \mathrm{~g} / \text { day of olive oil. } 15 \text { to } \\
18 \text { months of treatment }\end{array}$ & $\begin{array}{c}\text { Liver fat percentage } \\
\text { assessed by MRS and } \\
\text { biomarker scores for } \\
\text { liver fibrosis, } \\
\text { erythrocyte } \\
\text { enrichment } \\
\text { quantification with } \\
\text { DHA+EPA via gas } \\
\text { chromatography }\end{array}$ & $\begin{array}{l}\text { Trend to improve liver fat } \% \\
\text { with DHA+EPA } \\
\text { No improvement in liver } \\
\text { fibrosis scores }\end{array}$ & $\begin{array}{c}\text { Association between } \\
\text { erythrocyte DHA } \\
\text { enrichment with } \\
\text { DHA+EPA treatment and a } \\
\text { decrease of liver fat } \\
\text { percentage }\end{array}$ \\
\hline $\begin{array}{l}\text { Sanyal et al., } \\
\text { 2014. [205] }\end{array}$ & $\begin{array}{c}\text { Double-blind, } \\
\text { randomized, } \\
\text { placebo-controlled } \\
\text { trial }\end{array}$ & $\begin{array}{c}\text { Patients with NASH, } \\
\text { NAFLD activity scores } \geq \\
4, \text { with minimum scores } \\
\text { of } 1 \text { for steatosis and } \\
\text { inflammation, along with } \\
\text { either ballooning or at } \\
\text { least stage 1a fibrosis. } \\
n=243\end{array}$ & $\begin{array}{c}\text { Subjects were randomly } \\
\text { assigned to groups given } \\
\text { placebo }(n=75), \text { low- dosage } \\
\text { EPA-E }(1800 \mathrm{mg} / \mathrm{d} ; \mathrm{n}=82) \text {, or } \\
\text { high-dosage EPA-E } \\
(2700 \mathrm{mg} / \mathrm{d} ; n=86) \text { for } \\
12 \text { months }\end{array}$ & $\begin{array}{l}\text { The primary end } \\
\text { point: NAFLD } \\
\text { activity score } \leq 3, \\
\text { without worsening of } \\
\text { fibrosis; or a decrease } \\
\text { in NAFLD activity } \\
\text { score by } \geq 2 \text { with } \\
\text { contribution from }>1 \\
\text { parameter, without } \\
\text { worsening of fibrosis. } \\
\text { Liver enzymes, IR, } \\
\text { adiponectin, keratin } \\
18, \text { hs-CRP, or } \\
\text { hyaluronic acid were } \\
\text { measured as well }\end{array}$ & $\begin{array}{c}\text { No effects of EPA-E on } \\
\text { steatosis, inflammation, } \\
\text { ballooning, or fibrosis scores. } \\
\text { No effects on levels of liver } \\
\text { enzymes, IR, adiponectin, } \\
\text { keratin 18, hs-CRP, or } \\
\text { hyaluronic acid. } \\
\text { High-dosage EPA-E: } \downarrow \text { levels } \\
\text { of TG }\end{array}$ & $\begin{array}{l}\text { In a phase } 2 \text { trial, EPA-E } \\
\text { had no significant effect on } \\
\text { the histologic features of } \\
\text { NASH. EPA-E reduced } \\
\text { subjects' levels of } \\
\text { triglyceride compared with } \\
\text { placebo, without any } \\
\text { increase in serious adverse } \\
\text { events }\end{array}$ \\
\hline
\end{tabular}


Table 3. Cont

\begin{tabular}{|c|c|c|c|c|c|c|}
\hline Reference & Study Design & Population & Intervention & $\begin{array}{c}\text { Outcome } \\
\text { Measurements }\end{array}$ & Results & Comments \\
\hline $\begin{array}{l}\text { Li et al., } \\
\text { 2015. [206] }\end{array}$ & $\begin{array}{c}\text { Randomized } \\
\text { placebo-controlled } \\
\text { trial }\end{array}$ & $\begin{array}{c}\text { Patients diagnosed with } \\
\text { NASH Mean age: } 51 \\
\text { years old. Mean BMI: } \\
27.9 \mathrm{~kg} / \mathrm{m}^{2}\end{array}$ & $\begin{array}{l}\text { Intervention group }(n=39) \text { : } \\
50 \text { mL of PUFA with } 1: 1 \text { Ratio } \\
\text { of EPA and DHA added into } \\
\text { daily diet placebo: saline } \\
(n=39) \text {. Duration of } \\
\text { treatment: } 6 \text { months }\end{array}$ & $\begin{array}{l}\text { Liver enzymes, lipid } \\
\text { profile, markers of } \\
\text { inflammation and } \\
\text { oxidation, and } \\
\text { histological changes } \\
\text { by biopsy }\end{array}$ & $\begin{array}{c}\text { Liver function was } \\
\text { significantly improved: } \\
\downarrow \text { ALT / AST } \\
\downarrow \text { TG } \\
\downarrow \text { Total Cholesterol } \\
\downarrow \text { CRP (inflammation) } \\
\downarrow \text { MDA (oxidation) } \\
\downarrow \text { fibrotic parameters }\end{array}$ & $\begin{array}{l}6 \text { months of n-3 PUFA } \\
\text { therapy is beneficial for } \\
\text { improving NASH }\end{array}$ \\
\hline $\begin{array}{l}\text { Argo et al., } \\
\text { 2015. [207] }\end{array}$ & $\begin{array}{c}\text { Double-blind, } \\
\text { randomized, } \\
\text { placebo-controlled } \\
\text { trial }\end{array}$ & $\begin{array}{l}\text { Patients } 34 \text { subjects with } \\
\text { biopsy-proven NASH; } \\
\text { Mean age: } 47 \mathrm{y} \\
\text { Mean BMI: } 32.5 \mathrm{~kg} / \mathrm{m}^{2}\end{array}$ & $\begin{array}{c}\text { Oral supplementation of n-3 } \\
\text { PUFA } 3000 \text { mg/d (each } \\
1000 \mathrm{mg} \text { capsule contains } \\
35 \% \text { EPA, 25\% DHA and } 10 \% \\
\text { other n-3 PUFA), vs placebo } \\
\text { (soybean oil). } \\
n=17 \text { per group } \\
1 \text { year of treatment }\end{array}$ & $\begin{array}{c}\text { Liver biopsy, } \\
\text { Abdominal MRI for } \\
\text { quantitative } \\
\text { assessment of hepatic } \\
\text { fat, AST, ALT, total } \\
\text { cholesterol, LDL and } \\
\text { HDL cholesterol, and } \\
\text { TGs. FFAs, insulin, } \\
\text { and glucose levels }\end{array}$ & $\begin{array}{c}\text { No differences for the } \\
\text { primary end point of NASH } \\
\text { activity score (NAS) } \\
\text { reduction. } \\
\text { In n-3 PUFA-treated subjects: } \\
\downarrow \text { in liver fat content by MRI } \\
\text { (among subjects with } \\
\text { increased or stable weight) }\end{array}$ & $\begin{array}{c}\text { Treatment did not exert } \\
\text { beneficial effects towards } \\
\text { hepatic histological } \\
\text { improvement in NASH } \\
\text { patients }\end{array}$ \\
\hline $\begin{array}{l}\text { Qin et al., } \\
\text { 2015. [208] }\end{array}$ & $\begin{array}{l}\text { A double-blind } \\
\text { randomized } \\
\text { Placebo-controlled } \\
\text { clinical trial }\end{array}$ & $\begin{array}{c}\text { Patients with NAFLD } \\
\text { associated with } \\
\text { hyperlipidemia, Mean } \\
\text { age: } 44.3 \pm 10.9 \text { and } 46.0 \\
\pm 10.6 \text { y for placebo or } \\
\text { treated group } \\
\text { Mean BMI: } 26.0 \pm 2.8 \text { and } \\
26.4 \pm 3.9 \mathrm{~kg} / \mathrm{m}^{2}, \\
\text { respectively. } n=70\end{array}$ & $\begin{array}{l}\text { Randomly assigned to } \\
\text { consume fish oil }(n=36, \\
4 \mathrm{~g} / \mathrm{d}) \text { or corn oil capsules } \\
(n=34,4 \mathrm{~g} / \mathrm{d}) \text { for } 3 \text { months }\end{array}$ & $\begin{array}{l}\text { Blood levels of lipids, } \\
\text { glucose and insulin, } \\
\text { liver enzymes, and } \\
\text { cytokines at baseline } \\
\text { and the end of the } \\
\text { study were measured }\end{array}$ & $\begin{array}{c}\text { Fish oil group: } \\
\downarrow \text { total cholesterol, } \downarrow \text { TG, } \\
\downarrow \text { apolipoprotein B } \\
\downarrow \text { glucose, } \\
\downarrow \text { ALT } \\
\downarrow \text { GGT } \\
\uparrow \text { Adiponectin } \\
\downarrow \text { TNF- } \alpha \\
\downarrow \text { LTB4, } \\
\downarrow \text { FGF21, } \\
\downarrow \text { CK-18/M30 } \downarrow \text { PGE2 }\end{array}$ & $\begin{array}{l}\text { These findings suggest that } \\
\text { fish oil can benefit } \\
\text { metabolic abnormalities } \\
\text { associated with NAFLD }\end{array}$ \\
\hline $\begin{array}{l}\text { Dasarathy et al., } \\
\text { 2015. [209] }\end{array}$ & $\begin{array}{c}\text { Double-blind, } \\
\text { randomized, } \\
\text { placebo-controlled } \\
\text { trial }\end{array}$ & $\begin{array}{c}\text { Patients with NAFLD } \\
\text { and NASH diagnosed by } \\
\text { liver biopsy, Mean age: } \\
50 \text { y. Mean BMI: } 35 \mathrm{~kg} / \mathrm{m}^{2} . \\
\quad n=37\end{array}$ & $\begin{array}{l}\text { n-3 PUFA group: oral } \\
\text { supplementation of } 2160 \mathrm{mg} \\
\text { of EPA and } 1440 \text { mg of DHA. } \\
(n=19) \text { and Placebo group } \\
(n=18) \text { using corn oil } \\
\text { supplementation } \\
\text { Duration: } 48 \text { months }\end{array}$ & $\begin{array}{l}\text { Primary endpoints: } \\
\text { assess the } \\
\text { improvement of } 2 \\
\text { points in the NAFLD } \\
\text { activity score by liver } \\
\text { biopsy. } \\
\text { Secondary endpoints: } \\
\text { changes in liver } \\
\text { enzymes, IR, fasting } \\
\text { glucose, and HbA1C }\end{array}$ & $\begin{array}{l}\text { No differences between } \\
\text { groups in BMI, serum } \\
\text { transaminases, diabetes } \\
\text { control, histological } \\
\text { evaluation of NAFLD activity } \\
\text { score and individual } \\
\text { components }\end{array}$ & $\begin{array}{l}\text { N-3 PUFA supplementation } \\
\text { showed no beneficial } \\
\text { effects in NASH patients } \\
\text { with diabetes }\end{array}$ \\
\hline
\end{tabular}


Table 3. Cont.

\begin{tabular}{|c|c|c|c|c|c|c|}
\hline Reference & Study Design & Population & Intervention & $\begin{array}{c}\text { Outcome } \\
\text { Measurements }\end{array}$ & Results & Comments \\
\hline $\begin{array}{l}\text { Nogueira et al., } \\
2016 \text { [210] }\end{array}$ & $\begin{array}{c}\text { Double-blind, } \\
\text { randomized, } \\
\text { placebo-controlled } \\
\text { trial }\end{array}$ & $\begin{array}{c}\text { Men and women with a } \\
\text { proven histological } \\
\text { diagnosis of NASH. } \\
\text { Mean age: } 53.9 \pm 1.8 \text { and } \\
52.5 \pm 7.2 \text { y for placebo } \\
\text { group and n-3 PUFA } \\
\text { group. Mean BMI: } \\
30.3 \pm 4.4 \text { and } 31.1 \pm 4.6 \\
\text { respectively. } \\
n=50\end{array}$ & $\begin{array}{c}\text { n-3 PUFA group: } 3 \text { capsules } \\
\text { (0.945 g in total per day, } 64 \% \\
\text { ALA, } 16 \% \text { EPA, and } 21 \% \\
\text { DHA). }(n=27) \\
\text { Placebo group: } \\
3 \text { capsules of mineral oil } \\
(\mathrm{n}=23) . \\
\text { Duration: } 6 \text { months }\end{array}$ & $\begin{array}{c}\text { Primary endpoints: } \\
\text { Plasma fatty acids } \\
\text { (ALA, EPA, DHA and } \\
\text { AA), NAS. } \\
\text { Secondary endpoints: } \\
\text { serum TG, AST, ALT, } \\
\text { GGT, fasting } \\
\text { lipid profile, fasting } \\
\text { glucose, } \\
\text { anthropometric } \\
\text { parameters, or plasma } \\
\text { levels of IL-6 at } \\
\text { baseline and at } \\
\text { endpoint, }\end{array}$ & $\begin{array}{c}\text { n-3 PUFA group: } \\
\uparrow \text { plasma ALA and EPA. NAS } \\
\text { correlated with } \uparrow \text { plasma ALA. } \\
\downarrow \text { TG } \\
\text { Control group: } \\
\uparrow \text { plasma DHA and EPA, NAS } \\
\text { correlated with } \uparrow \text { plasma DHA } \\
\text { and EPA }\end{array}$ & $\begin{array}{l}\text { No significant changes } \\
\text { were observed on liver } \\
\text { histology in the n-3 PUFA } \\
\text { or placebo group }\end{array}$ \\
\hline $\begin{array}{l}\text { Tobin et al., } \\
2018 \text { [211] }\end{array}$ & $\begin{array}{c}\text { Double-blind, } \\
\text { randomized, } \\
\text { placebo-controlled } \\
\text { trial }\end{array}$ & $\begin{array}{c}\text { Patients with previously } \\
\text { diagnosed NAFLD } \\
\text { (hepatic steatosis stage). } \\
\text { Mean age; } 55.1 \pm 10.9 \text { and } \\
55.3 \pm 13.3 \text { y for placebo } \\
\text { and n-3 PUFA MF4637 } \\
\text { group } \\
\text { Mean BMI; } 32.4 \pm 5.0 \text { and } \\
\text { 32.1 } \pm 4.8 \text {. respectively. } \\
n=176\end{array}$ & $\begin{array}{c}\text { n-3 PUFA group: oral } \\
\text { supplementation of } 3 \mathrm{~g} \\
\text { capsule (1380 } \mathrm{g} \text { of EPA and } \\
1140 \mathrm{~g} \text { of DHA) }(n=87) \\
\text { Placebo group: oral } \\
\text { supplementation of } 3 \mathrm{~g} \text { olive } \\
\text { oil capsule }(n=89) \\
\text { Duration }=24 \text { weeks }\end{array}$ & $\begin{array}{l}\text { n-3 PUFA index, n-6 } \\
\text { PUFA: n-3 PUFA } \\
\text { ratio, quantitative } \\
\text { measurements of RBC } \\
\text { EPA and DHA at the } \\
\text { baseline and the } \\
\text { endpoint, liver fat } \\
\text { content measured by } \\
\text { MRI }\end{array}$ & $\begin{array}{c}\text { n-3 PUFA group: } \\
\uparrow n-3 \text { PUFA index and } \\
\uparrow \text { absolute values of RBC EPA } \\
\text { and DHA, } \downarrow \text { RBC n-6: n-3 } \\
\text { ratio } \\
\downarrow \text { liver fat content in both } \\
\text { groups }\end{array}$ & $\begin{array}{c}\text { No significant differences } \\
\text { in fat liver were found } \\
\text { between n-3 PUFA and } \\
\text { placebo group }\end{array}$ \\
\hline
\end{tabular}


In 2006, Capanni et al. [200] carried out the first clinical trial in humans to test the efficacy of long-term supplementation of n-3 PUFA in NAFLD. This study in patients with NAFLD confirmed by US showed that oral intake of n-3 PUFA (EPA and DHA in a 0.9/1.5 ratio) did not change BMI, but significantly decreased serum TG and glucose levels in parallel with a reduction in arachidonate and n-6/n-3 ratio. Concerning liver function, the study also revealed that the n-3 PUFA group exhibited lower levels of circulating AST, ALT, and GGT. Moreover, the US and duplex Doppler assays revealed that n-3 PUFA supplementation significantly improved liver echo-texture with a regression of hepatic brightness and higher Doppler perfusion index (DPI), which indicates an improvement in liver blood flow due to intrahepatic fat reduction [200].

The randomized/open-label trial by Spadaro et al. [111] showed similar patterns to the Capanni et al. study [200]. Patients with proven NAFLD by US followed dietary recommendations in concordance with the American Heart Association (AHA) guidelines, with a caloric restriction of $25-30 \mathrm{kcal} / \mathrm{kg}$ per day for 6 months. The group supplemented with n-3 PUFA ( $1 \mathrm{~g}$ twice a day) showed decreased circulating levels of TG and increased HDL levels. Moreover, in the n-3 PUFA group, ALT and GGT serum levels were markedly decreased. A complete steatosis regression was observed in $33.4 \%$ of patients, and an overall reduction in $50 \%$, suggesting a beneficial effect of long-term n-3 PUFA supplementation to reduce fatty liver. n-3 PUFA supplementation also reduced the inflammatory marker TNF- $\alpha$ and the IR index assessed by HOMA-IR. A correlation between both factors was observed [212]. Thus, TNF- $\alpha$ can influence insulin receptor phosphorylation, altering its tyrosine kinase activity and therefore inhibiting insulin receptor-initiated signals in hepatocytes [213]. High levels of TNF- $\alpha$ can initiate intracellular signaling which may lead to caspase activation and apoptosis in hepatocytes, casing inflammation and tissue fibrosis [214,215]. Therefore, by reducing TNF- $\alpha$ and inflammation, n-3 PUFA can protect hepatocytes from these damages [214]. The hyperinsulinemia condition that often occurs during IR can affect SREBP-1, a lipogenic transcription factor, which regulates lipid homeostasis by controlling a wide range of enzymes [216], enhancing fatty acid synthesis and accelerating TG accumulation [217].

Zhu et al. [201] conducted a randomized placebo-controlled trial using seal oil as the source of n-3 PUFA in patients with NAFLD associated with hyperlipidemia. Liver steatosis was diagnosed and monitored by US. Oral supplementation of n-3 PUFA ( $2 \mathrm{~g}$, three times a day) lasted for 24 weeks. No significant changes were observed in body weight and fasting blood glucose. Interestingly, total symptom scores, ALT and TG levels were significantly reduced in the intervention as compared with the placebo group. Moreover, the US revealed that at the end of treatment, $19.70 \%$ of the n-3 PUFA supplemented patients showed a normal liver echopattern and that $53.03 \%$ of them had an overall fatty liver regression. By contrast, in the placebo group only $7.35 \%$ of patients achieved complete regression. Seal oils have a different PUFA composition than fish oils, which have been mainly used in trials in humans. In fishes, EPA and DHA are positioned in sn-2, while in marine mammals, these fatty acids are found mainly at the sn- 1 and sn-3 positions of triglycerides. This study suggests that administration of seal oils rich in n-3 PUFA seems to be also efficient in combination with an energy restricted diet in treating patients with NAFLD associated with hyperlipidemia [201].

Sofi et al. [203] conducted a randomized study in subjects with NAFLD (BMI mean of $29.3 \mathrm{~kg} / \mathrm{m}^{2}$ ), in which subjects received $6.5 \mathrm{~mL} /$ day of olive oil enriched with n-3 PUFA ( $0.83 \mathrm{~g}$ n-3 PUFA, of which $0.47 \mathrm{~g}$ was EPA and $0.24 \mathrm{~g}$ was DHA), or the same dose of unenriched olive oil for 12 months. No specific diet was recommended, but food habits and estimation of nutrients and calorie intake were recorded; physical activity was also recorded by questionnaires and classified as light or absent, moderate, or intense. After a year of treatment, no significant BMI differences were found, while hepatic and lipid parameters were improved in the subjects receiving the n-3 PUFA-enriched olive oil. Indeed, ALT, AST, and GGT decreased by $40.4 \%, 35.3 \%$ and $27.2 \%$ respectively, and serum TG levels reduced by $19.2 \%$. Contrariwise, a predominant increase of HDL-C and adiponectin by $36.1 \%$ and $30.2 \%$ was observed in the intervention group. Moreover, the echo-Doppler test revealed an increase of $26.7 \%$ in DPI in the group consuming the n-3 PUFA-enriched olive oil [203]. 
The WELCOME study (Wessex Evaluation of fatty Liver and Cardiovascular markers in NAFLD with OMacor thErapy) evaluated whether the supplementation with highly purified n-3 PUFA could exert a more beneficial effect on NAFLD [204]. Overweight/obese patients diagnosed with NAFLD received $4 \mathrm{~g}$ /day of placebo (olive oil) or Omacor (1g contains $460 \mathrm{mg}$ of EPA and $380 \mathrm{mg}$ of DHA as ethyl esters) for 15 to 18 months. Liver fat percentage was evaluated by MRI and liver fibrosis by using two histological validated scores, which have different sensitivities in assessing grade of severity in steatosis. Erythrocyte enrichment with EPA+DHA was used to validate the adherence to intervention in the Omacor group and monitor contamination with DHA and EPA in the placebo group. The study revealed that n-3 PUFA supplementation did not improve fibrosis scores, while there was a trend to reduce liver fat in these patients. Regression analysis revealed an independent association between the reduction in the percentage of liver fat and DHA enrichment [204].

Qin et al. [208] carried out a double-blind placebo-controlled trial in patients with NAFLD (BMI 26.0), in which subjects received $4 \mathrm{~g} /$ day of placebo (corn oil) or fish oil capsules (with a total daily amount of $728 \mathrm{mg}$ of EPA and $516 \mathrm{mg}$ of DHA) for 3 months. After intervention, the fish oil group exhibited higher circulating levels of EPA and DHA, while a reduction was found in serum levels of glucose, TG, total cholesterol, and apolipoprotein B. Fish oil supplementation also reduced markers of inflammation such as TNF- $\alpha$, leukotriene B4, and prostaglandin E2, while it increased adiponectin, an anti-inflammatory adipokine. In this study, the authors, did not carry out US or MRI to estimate NAFLD, but several circulating markers of NAFLD were evaluated. After treatment, a significant decrease was induced by fish oil supplementation on ALT, GGT, FGF21, and cytokeratin 18 fragment M30. Increased levels of FGF21 have been found in patients with NAFLD, which is associated with chronic inflammation and considered as a result of a FGF21-resistant state [218].

Recently, Tobin et al. [211] conducted a double-blind randomized placebo-controlled study in subjects with NAFLD to evaluate the efficacy of an n-3 PUFA medical food (omega- 3 concentrate MF4637) provided as 3 capsules of $1 \mathrm{~g}$ per day (each capsule containing marine-sourced EPA and DHA as ethyl esters, $460 \mathrm{mg}$ and $380 \mathrm{mg}$, respectively) for 24 weeks. The high concentrate omega-3 intake significantly increased the omega-3 index and absolute values of red blood cells (RBC) EPA and DHA, and decreased the RBC n-6: n-3 PUFA ratio. ALT, AST, and GGT decreased in the placebo group but not in the group with the omega-3 concentrate. Liver fat content evaluated by magnetic resonance imaging-proton density fat fraction (MRI-PDFF) significantly decreased in both groups, but no differences were found between groups.

Li et al. [206] performed a randomized, but not blinded trial in overweight/obese participants diagnosed with NASH. The intervention group received n-3 PUFA treatment (50 mL of PUFA with 1:1 ratio of EPA and DHA in the daily diet), while the control group received saline solution for 6 months. Participants were advised to follow a low-fat and low-carbohydrate diet with moderate physical activity for $30 \mathrm{~min}$ for at least 5 times a week. Inflammation and oxidative parameters, C-reactive protein (CRP), MDA, as well as fibrotic parameters, type IV collagen and Procollagen-III-peptide (P-III-P) were significantly higher in patients with NASH. After 6 months of intervention with n-3 PUFA, liver function was generally improved, indicated by a reduction in ALT, AST, TG, total cholesterol, CRP, MDA, and type IV collagen and P-III-P.

On the other hand, Argo et al. [207] carried out a double-blind, randomized, placebo-controlled trial in obese subjects diagnosed with NASH in which the intervention group received $3000 \mathrm{mg} /$ day of n-3 PUFA (each capsule of $1000 \mathrm{mg}$ contained 70\% total n-3 PUFA in the form of triglycerides: $35 \%$ of EPA, $25 \%$ of DHA and $10 \%$ of other n-3 PUFA). Patients were advised to follow a hypocaloric diet (500-1000 kcal less than estimated for age- and weight-based basal metabolic rate) with less than $30 \%$ of fat content and $150 \mathrm{~min}$ of aerobic exercise per week. Serum transaminases, lipid profile, IR, and fasting glucose were determined at the beginning and at the end of the study, liver biopsy and MRI were applied to evaluate liver fat. Although a decrease in n-6/n-3 PUFA ratio in RBC as well as in liver fat reduction were noted in n-3 PUFA-treated patients, no significant changes of NASH were found in any group [207]. 
A study by Dasarathy et al. [209] was carried out with a double-blind placebo-controlled trial in diabetic patients with NASH diagnosed by liver biopsy. Patients received a placebo (corn oil) or n-3 PUFA (2160 mg of EPA and $1440 \mathrm{mg}$ of DHA daily) for 48 weeks. Outcomes revealed no significant changes in body weight or body composition. Liver steatosis and NAS were improved while lobular inflammation worsened in the placebo group; however, there was no significant change in any of the histological measures in the n-3 PUFA group. These outcomes suggest that n-3 PUFA supplementation did not provide any benefit over the placebo in NASH patients with diabetes. The authors reported that the effects of n-3 PUFA on histology and IR were inferior to the placebo.

Nogueira et al. [210] carried out a double-blind, placebo-controlled clinical trial in patients with NASH. The n-3 PUFA supplemented group received a total amount of $0.945 \mathrm{~g}$ of n-3 PUFA (64\% ALA, $16 \% \mathrm{EPA}$, and 21\% DHA) for 6 months. Comparison between the final and basal liver histopathologic scores showed no significant changes between the n-3 PUFA and the placebo groups regarding hepatocellular ballooning, liver steatosis, or fibrosis. Surprisingly, the lobular inflammation was improved in the placebo group in parallel with an increase in plasma EPA and DHA levels, suggesting an off-protocol intake of n-3 PUFAs.

All the previous studies have been carried out with different n-3 PUFA formulations containing both EPA and DHA. Only two studies have analyzed the effects of purified EPA on NASH patients.

Thus, Tanaka et al. [202] carried out a pilot trial to evaluate the efficacy of highly purified EPA $(2700 \mathrm{mg} /$ day) on 23 biopsy-proven NASH patients for 12 months. No changes were found in body weight, blood glucose, insulin, or adiponectin. However, circulating levels of ALT were significantly decreased. Post-treatment liver biopsies were obtained from 7 patients, and improved hepatic steatosis and fibrosis, hepatocyte ballooning, and lobular inflammation were found in 6 patients. These findings suggest that highly purified EPA treatment could be efficient for NASH treatment, probably due to its anti-inflammatory and antioxidant properties. To better characterize this finding, Sanyal et al. [205] carried out a multicenter, prospective, double-blind, randomized, placebo-controlled trial with EPA-Ethyl ester ( $1800 \mathrm{mg} /$ day or $2700 \mathrm{mg} /$ day) for 12 months in 243 patients with diagnosed $\mathrm{NASH}$. The highest dose of EPA reduced the circulating levels of TG, but no significant changes on liver enzymes, IR, adiponectin, keratin 18, CRP, or hyaluronic acid were found after EPA supplementation. Moreover, EPA did not have any relevant effect on histologic features of NASH.

From the eight trials analyzing effects of n-3 PUFA on NAFLD patients, five suggested beneficial effects on some biomarkers of liver steatosis such as a decrease of ALT, AST or GGT, or a reduction of fat liver percentage or biomarkers of metabolic abnormalities associated with NAFLD [111,200,201,203,208]. However, the range of doses used in the studies, the type of formulation of n-3 PUFA (fish oil, seal oil, olive oil enriched with n-3 PUFA), the ratio EPA/DHA, if they are in the form of TG or ethyl esters, etc.), as well as the duration of the trials ( 3 to 12 months) were widely different among the trials. Therefore, it is difficult to provide a concluding recommendation about the most effective dose and duration of the trial. Moreover, two trials using higher doses of EPA and DHA ( $>3 \mathrm{~g} /$ day) during a longer period of time (15 to 48 months) did not find any significant changes on serum transaminases or NAFLD activity scores $[204,209]$. In the same way, a recent trial testing the effects of $3 \mathrm{~g}$ of a high concentrate omega-3 medical food (1.38 g EPA and 1.14 DHA) has not found significant differences with the placebo group in NAFLD patients [211]. The inconsistent outcomes of the different trials may be also related with the heterogeneity of participants within the spectrum of NAFLD (lean, overweight, or obese, with or without other metabolic complications such as type 2 diabetes or severe dyslipidemia, as well as the severity of the liver steatosis). Trials also differ in the methodology to evaluate the degree of liver disease in NAFLD and NASH patients.

Six studies have analyzed the potential beneficial effects of n-3 PUFA supplementation on NASH diagnosed patients. Two studies suggested that n-3 PUFA supplementation could be useful for improving NASH [202,206]. However, these studies have some limitations. In one of them, the intervention group received $50 \mathrm{~mL}$ of PUFA/day with a ratio 1:1 of EPA:DHA added into the diet, but the composition of the PUFA preparation and the exact amount of EPA and DHA received daily by 
the patients was not indicated. Moreover, the control group received saline and no other oil with similar calorie supply. Patients of both groups reduced their BMI after the intervention, probably in response to the dietary recommendations and modest physical activity. The decrease in BMI tended to be higher in the PUFA group, and therefore the beneficial effects observed could be secondary to a higher reduction of body weight or fat mass, which was not considered in the interpretation of the data [206]. The other study was a pilot trial without a placebo group, which suggested that the administration of highly purified EPA can improve NASH features [202]. However, a further double-blind placebo-controlled trial providing the same dose of highly purified EPA (2700 mg/day) also for 12 months did not find any significant change in steatosis or fibrosis in NASH patients [205]. Similarly, two double-blind placebo-controlled trials using high doses of n-3 PUFA ( $\geq 3000 \mathrm{mg} /$ day) for 12 to 48 months did not observe any significant improvement on the histological features of NASH [207,209]. In the same way, Nogueira et al. [210] described that the supplementation with n-3 PUFAs from a flaxseed/fish oil mixture did not improve liver histology in NASH patients when compared to placebo. Therefore, the effectiveness of n-3 PUFA to attenuate severe NAFLD or NASH markers including liver fibrosis or inflammation is still unclear.

Some studies support differential effects for EPA and DHA [219]. Thus, trials in children and adolescent have suggested that DHA was more effective in reducing liver fat and markers of liver fibrosis [220,221]. Moreover, the study of Scorletti et al. [204] found an independent association between reduced liver fat percentage and erythrocyte DHA enrichment (but not with erythrocyte EPA enrichment). However, there are no trials in adults evaluating the efficacy of highly purified DHA in patients with NAFLD/NASH.

In conclusion, the trials performed to date suggest that n-3 PUFA supplementation maybe effective in the early stages of NAFLD, but not in patients with more severe NAFLD or NASH. Further randomized controlled trials are needed to evaluate the efficacy of DHA on these conditions. Also, it is important to better characterize if the n-3 PUFA supplementation is more effective when combined with a calorie restricted diet or exercise.

\section{Conclusions and Future Perspectives}

Oxidative stress has been related with the development of several age-related conditions including cardiovascular and neurodegenerative diseases, and plays a key role in combination with inflammation and lipotoxicity in the progression of NAFLD to NASH. The outcomes derived from the different trials addressing the potential usefulness of n-3 PUFA supplementation to attenuate oxidative stress are controversial (with beneficial, neutral, or even negative actions depending on the oxidative-stress biomarker measured). Those articles that observed a beneficial effect on oxidative stress suggested that the effect could be related to the immuno-modulatory and anti-inflammatory properties of n-3 PUFA and to their ability to increase antioxidant enzymes, which could contribute to reduce the generation of ROS and other oxidative stress agents. However, the heterogeneity in population participating in the randomized trials, the differences in treatment duration, doses, as well as in methods to assess oxidative stress make difficult to conclude about the effectiveness of n-3 PUFA to reduce oxidative stress during aging.

Concerning NAFLD, the systematic review of randomized controlled trials strongly suggests that n-3 PUFA supplementation may be an effective option to decrease liver fat and circulating enzymes related to liver injury in adult patients with NAFLD. However, the effectiveness of n-3 PUFA to attenuate more severe NAFLD or NASH markers including liver fibrosis is still inconclusive. Therefore, it is difficult to provide a recommendation about the proper doses and formulas (EPA vs DHA or EPA/DHA ratio) to attenuate the progression or to reduce NAFLD/NASH. Large-scale, well-designed randomized controlled trials are needed to better characterize the efficacy of n-3 PUFA for oxidative stress and NAFLD/NASH treatment in adults. Future research should also focus on analyzing the differences in bioavailability and effects of different formulations of n-3 PUFA, including re-esterified TG, ethyl ester, carboxylic acids, and phospholipids. 
Another important issue is the evidence about the heterogeneity in the response to n-3 PUFA supplementation within-population. Genetic background may clearly influence this differential responsiveness. For example, PNPLA3 rs738409 underlies the response to a variety of treatments in NAFLD [222]. Consequently, future efforts should be paid to the identification of genetic/epigenetic signatures involved in the response to treatments to warrant a personalized, precise medicine in NAFLD.

Metabolomics and lipidomics studies are also needed to better understand the alterations in the metabolome that occur during aging-related pathologies, including NAFLD, as well as the changes in the metalolomic/lipidomic signatures induced by n-3 PUFA, which could contribute to explain the beneficial actions of these fatty acids through the production of SPMs or other metabolites. In this way a recent study using a proteomic approach in liver biopsias and lipidomic analysis of plasma has found that n-3 PUFA supplementation improve markers of lipogenesis, ER stress and mitochondrial function in patients with NASH [223].

Trials should also consider analyzing gut microbiota changes during the aging process as well as their modulation by dietary factors including n-3 PUFA and to characterize the mechanisms linking the relationship between the modifications of gut microbiota composition and the evolution of oxidative stress and liver pathology.

Author Contributions: All authors have significantly contributed to the writing and the critical revision of the different parts of this review manuscript. Conceptualization and methodology: J.Y., M.F.-G., J.A.M. and M.J.M.-A. Writing-Original Draft: J.Y., M.F.-G., L.M.-F., P.G.-M., A.P.-C., J.A.M. and M.J.M.-A. Writing Review and Editing: M.F.-G., J.Y., P.G.-M., M.J.M.-A. Funding Acquisition: M.J.M.-A. and J.A.M. All authors approved the final manuscript.

Funding: This research was funded by: Ministry of Economy, Industry and Competitiveness (MINECO-FEDER) of the Government of Spain (BFU2015-65937-R), Department of Health of the Navarra Government (67-2015) and CIBER Physiopathology of Obesity and Nutrition (CIBERobn), Carlos III Health Research Institute (CB12/03/30002). "Juan de la Cierva" Grant to M.F.-G. (IJCI-2016-30025). L.M.-F. was supported by an FPI predoctoral fellowship (Formación de Personal Investigador). The support from Nutrition Research Centre of the University of Navarra is also acknowledged.

Conflicts of Interest: The authors declare no conflict of interest.

\section{References}

1. Da Costa, J.P.; Vitorino, R.; Silva, G.M.; Vogel, C.; Duarte, A.C.; Rocha-Santos, T. A synopsis on aging-Theories, mechanisms and future prospects. Ageing Res. Rev. 2016, 29, 90-112. [CrossRef] [PubMed]

2. Kirkwood, T.B.L. Systems biology of ageing and longevity. Philos. Trans. R. Soc. B Biol. Sci. 2011, 366, 64-70. [CrossRef] [PubMed]

3. Picca, A.; Fanelli, F.; Calvani, R.; Mulè, G.; Pesce, V.; Sisto, A.; Pantanelli, C.; Bernabei, R.; Landi, F.; Marzetti, E. Gut Dysbiosis and Muscle Aging: Searching for Novel Targets against Sarcopenia. Mediat. Inflamm. 2018, 2018, 7026198. [CrossRef] [PubMed]

4. North, B.J.; Sinclair, D.A. The intersection between aging and cardiovascular disease. Circ. Res. 2012, 110, 1097-1108. [CrossRef] [PubMed]

5. Gunasekaran, U.; Gannon, M. Type 2 Diabetes and the Aging Pancreatic Beta Cell. Aging 2011, 3, 565-575. [CrossRef]

6. Querfurth, H.W.; LaFerla, F.M. Alzheimer's Disease. N. Engl. J. Med. 2010, 362, 329-344. [CrossRef]

7. He, F.; Zuo, L. Redox roles of reactive oxygen species in cardiovascular diseases. Int. J. Mol. Sci. 2015, 16, 27770-27780. [CrossRef]

8. Zuo, L.; Pannell, B.K. Redox characterization of functioning skeletal muscle. Front. Physiol. 2015, 6, 1-9. [CrossRef]

9. Birben, E.; Sahiner, U.M.; Sackesen, C.; Erzurum, S.; Kalayci, O. Oxidative stress and antioxidant defense. World Allergy Organ J. 2012, 5, 9-19. [CrossRef] [PubMed]

10. Harman, D. Aging: A Theory Based on Free Radical and Radiation Chemistry. J. Gerontol. 1956, 11, $298-300$. [CrossRef]

11. Pomatto, L.C.D.; Davies, K.J.A. Adaptive homeostasis and the free radical theory of ageing. Free Radic. Biol. Med. 2018, 124, 420-430. [CrossRef] [PubMed] 
12. Zsurka, G.; Peeva, V.; Kotlyar, A.; Kunz, W.S. Is there still any role for oxidative stress in mitochondrial DNA-dependent aging? Genes 2018, 9, 175. [CrossRef] [PubMed]

13. Kelley, N.S.; Yoshida, Y.; Erickson, K.L. Do n-3 polyunsaturated fatty acids increase or decrease lipid peroxidation in humans? Metab. Syndr. Relat. Disord. 2014, 12, 403-415. [CrossRef] [PubMed]

14. Lawson, J.A.; Rokach, J.; FitzGerald, G.A. Isoprostanes: Formation, analysis and use as indices of lipid peroxidation in vivo. J. Biol. Chem. 1999, 274, 24441-24444. [CrossRef] [PubMed]

15. Praticò, D.; MY Lee, V.; Trojanowski, J.Q.; Rokach, J.; Fitzgerald, G.A. Increased F2-isoprostanes in Alzheimer's disease: Evidence for enhanced lipid peroxidation in vivo. FASEB J. 1998, 12, 1777-1783. [CrossRef] [PubMed]

16. Chalasani, N.; Younossi, Z.; Lavine, J.E.; Diehl, A.M.; Brunt, E.M.; Cusi, K.; Charlton, M.; Sanyal, A.J. The diagnosis and management of non-alcoholic fatty liver disease: Practice Guideline by the American Association for the Study of Liver Diseases, American College of Gastroenterology, and the American Gastroenterological Association. Hepatology 2012, 55, 2005-2023. [CrossRef]

17. Romá-Mateo, C.; Seco-Cervera, M.; Ibáñez-Cabellos, J.S.; Pérez, G.; Berenguer-Pascual, E.; Rodríguez, L.R.; García-Giménez, J.L. Oxidative stress and the epigenetics of cell senescence: Insights from progeroid syndromes. Curr. Pharm. Des. 2018, 24, 4755-4770. [CrossRef]

18. Schmucker, D.L. Age-related changes in liver structure and function: Implications for disease? Exp. Gerontol. 2005, 40, 650-659. [CrossRef]

19. Benedict, M.; Zhang, X. Non-alcoholic fatty liver disease: An expanded review. World J. Hepatol. 2017, 9 , 715-732. [CrossRef]

20. Younossi, Z.M.; Koenig, A.B.; Abdelatif, D.; Fazel, Y.; Henry, L.; Wymer, M. Global epidemiology of nonalcoholic fatty liver disease-Meta-analytic assessment of prevalence, incidence, and outcomes. Hepatology 2016, 64, 73-84. [CrossRef]

21. Angulo, P. GI epidemiology: Nonalcoholic fatty liver disease. Aliment. Pharmacol. Ther. 2007, 25, 883-889. [CrossRef] [PubMed]

22. Williams, C.D.; Stengel, J.; Asike, M.I.; Torres, D.M.; Shaw, J.; Contreras, M.; Landt, C.L.; Harrison, S.A. Prevalence of nonalcoholic fatty liver disease and nonalcoholic steatohepatitis among a largely middle-aged population utilizing ultrasound and liver biopsy: A prospective study. Gastroenterology 2011, 140, 124-131. [CrossRef] [PubMed]

23. Hashimoto, E.; Taniai, M.; Tokushige, K. Characteristics and diagnosis of NAFLD/NASH. J. Gastroenterol. Hepatol. 2013, 28, 64-70. [CrossRef] [PubMed]

24. Vernon, G.; Baranova, A.; Younossi, Z.M. Systematic review: The epidemiology and natural history of non-alcoholic fatty liver disease and non-alcoholic steatohepatitis in adults. Aliment. Pharmacol. Ther. 2011, 34, 274-285. [CrossRef]

25. Bugianesi, E.; McCullough, A.J.; Marchesini, G. Insulin Resistance: A Metabolic Pathway to Chronic Liver Disease. Hepatology 2005, 42, 987-1000. [CrossRef] [PubMed]

26. Parekh, S.; Anania, F.A. Abnormal Lipid and Glucose Metabolism in Obesity: Implications for Nonalcoholic Fatty Liver Disease. Gastroenterology 2007, 132, 2191-2207. [CrossRef] [PubMed]

27. Chitturi, S.; Farrell, G.C.; Hashimoto, E.; Saibara, T.; Lau, G.K.; Sollano, J.D. Non-alcoholic fatty liver disease in the Asia-Pacific region: Definitions and overview of proposed guidelines. J. Gastroenterol. Hepatol. 2007, 22, 778-787. [CrossRef]

28. Bellentani, S. The epidemiology of non-alcoholic fatty liver disease. Liver Int. 2017, 37, 81-84. [CrossRef]

29. Koehler, E.M.; Schouten, J.N.L.; Hansen, B.E.; Van Rooij, F.J.A.; Hofman, A.; Stricker, B.H.; Janssen, H.L.A. Prevalence and risk factors of non-alcoholic fatty liver disease in the elderly: Results from the Rotterdam study. J. Hepatol. 2012, 57, 1305-1311. [CrossRef]

30. Souza, M.R.; Diniz Mde, F.; Medeiros Filho, J.E.; Araújo, M.S. Metabolic syndrome and risk factors for non-alcoholic fatty liver disease. Arq. Gastroenterol. 2012, 49, 89-96. [CrossRef]

31. Klair, J.S.; Yang, J.D.; Abdelmalek, M.F.; Guy, C.D.; Gill, R.M.; Yates, K.; Unalp Arida, A.; Lavine, J.E.; Clark, J.M.; Diehl, A.M.; et al. A longer duration of estrogen deficiency increases fibrosis risk among postmenopausal women with nonalcoholic fatty liver disease. Hepatology 2016, 64, 85-91. [CrossRef] [PubMed]

32. Vehmas, A.P.; Adam, M.; Laajala, T.D.; Kastenmüller, G.; Prehn, C.; Rozman, J.; Ohlsson, C.; Fuchs, H.; Hrabě de Angelis, M.; Gailus Durner, V.; et al. Liver lipid metabolism is altered by increased circulating estrogen to androgen ratio in male mouse. J. Proteom. 2016, 133, 66-75. [CrossRef] 
33. Panic, A.; Stanimirovic, J.; Obradovic, M.; Sudar Milovanovic, E.; Perovic, M.; Lackovic, M.; Petrovic, N.; Isenovic, E.R. Estradiol-mediated regulation of hepatic iNOS in obese rats: Impact of Src, ERK1/2, AMPK $\alpha$, and miR-221. Biotechnol. Appl. Biochem. 2018, 65, 797-806. [CrossRef]

34. Veronese, N.; Notarnicola, M.; Osella, A.R.; Cisternino, A.M.; Reddavide, R.; Inguaggiato, R.; Guerra, V.; Rotolo, O.; Zinzi, I.; Chiloiro, M.; et al. Menopause Does Not Affect Fatty Liver Severity in Women: A Population Study in a Mediterranean Area. Endocr. Metab. Immune Disord. Drug Targets 2018, 18, 513-521. [CrossRef] [PubMed]

35. Frith, J.; Jones, D.; Newton, J.L. Chronic liver disease in an ageing population. Age Ageing 2009, 38, 11-18. [CrossRef]

36. Wynne, H.A.; Cope, L.H.; Mutch, E.; Rawlins, M.D.; Woodhouse, K.W.; James, O.F.W. The effect of age upon liver volume and apparent liver blood flow in healthy man. Hepatology 1989, 9, 297-301. [CrossRef] [PubMed]

37. Tietz, N.W.; Shuey, D.F.; Wekstein, D.R. Laboratory values in fit aging individuals-Sexagenarians through centenarians. Clin. Chem. 1992, 38, 1167-1185. [PubMed]

38. Ericsson, S.; Eriksson, M.; Vitols, S.; Einarsson, K.; Berglund, L.; Angelin, B. Influence of age on the metabolism of plasma low density lipoproteins in healthy males. J. Clin. Investig. 1991, 87, 591-596. [CrossRef]

39. Bussel, J.B.; Berkowitz, R.L.; McFarland, J.G.; Lynch, L.; Chitkara, U. Influence of age on secretion of cholesterol and synthesis of bile acids by the liver. N. Engl. J. Med. 1985, 319, 1374-1378. [CrossRef]

40. Conlon, B.A.; Beasley, J.M.; Aebersold, K.; Jhangiani, S.S.; Wylie-Rosett, J. Nutritional management of insulin resistance in nonalcoholic fatty liver disease (NAFLD). Nutrients 2013, 11, 4093-4114. [CrossRef]

41. Day, C.P.; James, O.F.W. Steatohepatitis: A tale of two “hits"? Gastroenterology 1998, 114, 842-845. [CrossRef]

42. Paglialunga, S.; Dehn, C.A. Clinical assessment of hepatic de novo lipogenesis in non-alcoholic fatty liver disease. Lipids Health Dis. 2016, 15, 1-10. [CrossRef] [PubMed]

43. Gaggini, M.; Morelli, M.; Buzzigoli, E.; DeFronzo, R.A.; Bugianesi, E.; Gastaldelli, A. Non-alcoholic fatty liver disease (NAFLD) and its connection with insulin resistance, dyslipidemia, atherosclerosis and coronary heart disease. Nutrients 2013, 5, 1544-1560. [CrossRef] [PubMed]

44. Koliwad, S.K.; Streeper, R.; Monetti, M.; Cornelissen, I.; Chan, L.; Terayama, K.; Naylor, S.; Rao, M.; Hubbard, B.; Farese, R.V., Jr. DGAT1-dependent triacylglycerol storage by macrophages protects mice from diet-induced insulin resistance and inflammation. J. Clin. Investig. 2010, 120, 756-767. [CrossRef]

45. Yamaguchi, K.; Yang, L.; McCall, S.; Huang, J.; Yu, X.X.; Pandey, S.K.; Bhanot, S.; Monia, B.P.; Li, Y.X.; Diehl, A.M. Inhibiting triglyceride synthesis improves hepatic steatosis but exacerbates liver damage and fibrosis in obese mice with nonalcoholic steatohepatitis. Hepatology 2007, 45, 1366-1374. [CrossRef] [PubMed]

46. Amaro, A.; Fabbrini, E.; Kars, M.; Yue, P.; Schechtman, K.; Schonfeld, G.; Klein, S. Dissociation between intrahepatic triglyceride content and insulin resistance in familial hypobetalipoproteinemia. Gastroenterology 2010, 139, 149-153. [CrossRef]

47. Peverill, W.; Powell, L.W.; Skoien, R. Evolving concepts in the pathogenesis of NASH: Beyond steatosis and inflammation. Int. J. Mol. Sci. 2014, 15, 8591-8638. [CrossRef]

48. Leamy, A.K.; Egnatchik, R.A.; Young, J.D. Molecular mechanisms and the role of saturated fatty acids in the progression of non-alcoholic fatty liver disease. Prog. Lipid Res. 2013, 52, 165-174. [CrossRef]

49. Novo, E.; Busletta, C.; di Bonzo, L.V.; Povero, D.; Paternostro, C.; Mareschi, K.; Ferrero, I.; David, E.; Bertolani, C.; Caligiuri, A.; et al. Intracellular reactive oxygen species are required for directional migration of resident and bone marrow-derived hepatic pro-fibrogenic cells. J. Hepatol. 2011, 54, 964-974. [CrossRef]

50. Tilg, H.; Moschen, A.R. Evolution of Inflammation in Nonalcoholic Fatty Liver Disease: The Multiple Parallel Hits Hypothesis. Hepatology 2010, 52, 1836-1846. [CrossRef]

51. Cancello, R.; Tordjman, J.; Poitou, C.; Guilhem, G.; Bouillot, J.L.; Hugol, D.; Coussieu, C.; Basdevant, A.; Hen, A.B.; Bedossa, P.; et al. Increased Infiltration of Macrophages in Omental Adipose Tissue Is Associated with Marked Hepatic Lesions in Morbid Human Obesity. Diabetes 2006, 55, 1554-1561. [CrossRef] [PubMed]

52. Canfora, E.E.; Meex, R.C.R.; Venema, K.; Blaak, E.E. Gut microbial metabolites in obesity, NAFLD and T2DM. Nat. Rev. Endocrinol. 2019, 15, 261-273. [CrossRef] [PubMed]

53. Marra, F.; Svegliati-Baroni, G. Lipotoxicity and the gut-liver axis in NASH pathogenesis. J. Hepatol. 2018, 68, 280-295. [CrossRef] [PubMed] 
54. Amar, J.; Burcelin, R.; Ruidavets, J.B.; Cani, P.D.; Fauvel, J.; Alessi, M.C.; Chamontin, B.; Ferriéres, J. Energy intake is associated with endotoxemia in apparently healthy men. Am. J. Clin. Nutr. 2008, 87, 1219-1223. [CrossRef]

55. Samuel, B.S.; Shaito, A.; Motoike, T.; Rey, F.E.; Backhed, F.; Manchester, J.K.; Hammer, R.E.; Williams, S.C.; Crowley, J.; Yanagisawa, M.; et al. Effects of the gut microbiota on host adiposity are modulated by the short-chain fatty-acid binding G protein-coupled receptor, Gpr41. Proc. Natl. Acad. Sci. USA 2008, 105, 16767-16772. [CrossRef]

56. Vernia, P.; Marcheggiano, A.; Caprilli, R.; Frieri, G.; Corrao, G.; Valpiani, D. Short-chain fatty acid topical treatment in distal ulcerative colitis. Aliment. Pharmacol. Ther. 1995, 9, 309-313. [CrossRef]

57. Purohit, V.; Bode, J.C.; Bode, C.; Brenner, D.A.; Choudhry, M.A.; Hamilton, F.; Kang, Y.J.; Keshavarzian, A.; Rao, R.; Sartor, R.B.; et al. Alcohol, intestinal bacterial growth, intestinal permeability to endotoxin, and medical consequences: Summary of a symposium. Alcohol 2008, 42, 349-361. [CrossRef]

58. Zhu, L.; Baker, S.S.; Gill, C.; Liu, W.; Alkhouri, R.; Baker, R.D.; Gill, S.R. Characterization of gut microbiomes in nonalcoholic steatohepatitis (NASH) patients: A connection between endogenous alcohol and NASH. Hepatology 2013, 57, 601-609. [CrossRef]

59. Setshedi, M.; Wands, J.R.; Monte, S.M. Acetaldehyde adducts in alcoholic liver disease. Oxid. Med. Cell. Longev. 2010, 3, 178-185. [CrossRef]

60. Su, G.L. Lipopolysaccharides in liver injury: Molecular mechanisms of Kupffer cell activation. Am. J. Physiol. Gastrointest. Liver Physiol. 2002, 283, G256-G265. [CrossRef]

61. Jones, R.M.; Neish, A.S. Redox signaling mediated by the gut microbiota. Free Radic. Biol. Med. 2017, 105, 41-47. [CrossRef]

62. Gustot, T.; Lemmers, A.; Moreno, C.; Nagy, N.; Quertinmont, E.; Nicaise, C.; Franchimont, D.; Louis, H.; Devière, J.; Le Moine, O. Differential liver sensitization to toll-like receptor pathways in mice with alcoholic fatty liver. Hepatology 2006, 43, 989-1000. [CrossRef]

63. Mardinoglu, S.; Shoaie, M.; Bergentall, P.; Ghaffari, C.; Zhang, E.L.; Nielsen, F.B.J. The gut microbiota modulates host amino acid and glutathione metabolism in mice. Mol. Syst. Biol. 2015, 11, 834. [CrossRef]

64. Kojer, K.; Bien, M.; Gangel, H.; Morgan, B.; Dick, T.P.; Riemer, J. Glutathione redox potential in the mitochondrial intermembrane space is linked to the cytosol and impacts the Mia40 redox state. EMBO J. 2012, 31, 3169-3182. [CrossRef]

65. Morgan, B.; Ezerina, D.; Amoako, T.N.; Riemer, J.; Seedorf, M.; Dick, T.P. Multiple glutathione disulfide removal pathways mediate cytosolic redox homeostasis. Nat. Chem. Biol. 2013, 9, 119-125. [CrossRef]

66. Oliveira, C.P.; Stefano, J.T. Genetic polymorphisms and oxidative stress in non-alcoholic steatohepatitis (NASH): A mini review. Clin. Res. Hepatol. Gastroenterol. 2015, 39 (Suppl. 1), S35-S40. [CrossRef]

67. Cobbina, E.; Akhlaghi, F. Non-alcoholic fatty liver disease (NAFLD)_Pathogenesis, classification, and effect on drug metabolizing enzymes and transporters. Drug Metab. Rev. 2017, 49, 197-211. [CrossRef]

68. Dai, G.; Liu, P.; Li, X.; Zhou, X.; He, S. Association between PNPLA3 rs738409 polymorphism and nonalcoholic fatty liver disease (NAFLD) susceptibility and severity: A meta-analysis. Medicine 2019, 98, e14324. [CrossRef]

69. Li, Y.; Liu, S.; Gao, Y.; Ma, H.; Zhan, S.; Yang, Y.; Xin, Y.; Xuan, S. Association of TM6SF2 rs58542926 gene polymorphism with the risk of non-alcoholic fatty liver disease and colorectal adenoma in Chinese Han population. BMC Biochem. 2019, 20,3. [CrossRef]

70. Wang, J.K.; Feng, Z.W.; Li, Y.C.; Li, Q.Y.; Tao, X.Y. Association of tumornecrosis factor-gene promoter polymorphism at sites -308 and -238 with non-alcoholic fatty liver disease: A meta-analysis. J. Gastroenterol. Hepatol. 2012, 27, 670-676. [CrossRef]

71. Nelson, J.E.; Handa, P.; Aouizerat, B.; Wilson, L.; Vemulakonda, L.A.; Yeh, M.M.; Kowdley, K.V. Increased parenchymal damage and steatohepatitis in Caucasian non-alcoholic fatty liver disease patients with common IL1B and IL6 polymorphisms. Aliment. Pharmacol. Ther. 2016, 44, 1253-1264. [CrossRef] [PubMed]

72. Kurbatova, I.V.; Topchieva, L.V.; Dudanova, O.P. Gene TNF Polymorphism -308G>A (rs1800629) and Its Relationship with the Efficiency of Ursodeoxycholic Acid Therapy in Patients with Nonalcoholic Stetohepatitis. Bull. Exp. Biol. Med. 2017, 164, 181-185. [CrossRef] [PubMed]

73. Kiziltas, S.; Ata, P.; Colak, Y.; Mesçi, B.; Senates, E.; Enc, F.; Ulasoglu, C.; Tuncer, I.; Oguz, A. TLR4 gene polymorphism in patients with nonalcoholic fatty liver disease in comparison to healthy controls. Metab. Syndr. Relat. Disord. 2014, 12, 165-170. [CrossRef] 
74. Kapil, S.; Duseja, A.; Sharma, B.K.; Singla, B.; Chakraborti, A.; Das, A.; Ray, P.; Dhiman, R.K.; Chawla, Y. Genetic polymorphism in CD14 gene, a co-receptor of TLR4 associated with non-alcoholic fatty liver disease. World J. Gastroenterol. 2016, 14, 9346-9355. [CrossRef] [PubMed]

75. Nobili, V.; Donati, B.; Panera, N.; Vongsakulyanon, A.; Alisi, A.; Dallapiccola, B.; Valenti, L. A 4-polymorphism risk score predicts steatohepatitis in children with nonalcoholic fatty liver disease. J. Pediatr. Gastroenterol. Nutr. 2014, 58, 632-636. [CrossRef]

76. Al-Serri, A.; Anstee, Q.M.; Valenti, L.; Nobili, V.; Leathart, J.B.; Dongiovanni, P.; Patch, J.; Fracanzani, A.; Fargion, S.; Day, C.P.; et al. The SOD2 C47T polymorphism influences NAFLD fibrosis severity: Evidence from case-control and intra-familial allele association studies. J. Hepatol. 2012, 56, 448-454. [CrossRef]

77. Xu, Y.P.; Liang, L.; Wang, C.L.; Fu, J.F.; Liu, P.N.; Lv, L.Q.; Zhu, Y.M. Association between UCP3 gene polymorphisms and nonalcoholic fatty liver disease in Chinese children. World J. Gastroenterol. 2013, 19, 5897-5903. [CrossRef]

78. Aller, R.; De Luis, D.A.; Izaola, O.; González Sagrado, M.; Conde, R.; Alvarez, T.; Pacheco, D.; Velasco, M.C. Role of -55CT polymorphism of UCP3 gene on non-alcoholic fatty liver disease and insulin resistance in patients with obesity. Nutr. Hosp. 2010, 25, 572-576.

79. Hashemi, M.; Hoseini, H.; Yaghmaei, P.; Moazeni-Roodi, A.; Bahari, A.; Hashemzehi, N.; Shafieipour, S. Association of polymorphisms in glutamate-cysteine ligase catalytic subunit and microsomal triglyceride transfer protein genes with nonalcoholic fatty liver disease. DNA Cell Biol. 2011, 308, 569-575. [CrossRef]

80. Oliveira, C.P.; Stefano, J.T.; Cavaleiro, A.M.; Zanella Fortes, M.A.; Vieira, S.M.; Rodrigues Lima, V.M.; Santos, T.E.; Santos, V.N.; de Azevedo Salgado, A.L.; Parise, E.R.; et al. Association of polymorphisms of glutamate-cystein ligase and microsomal triglyceride transfer protein genes in non-alcoholic fatty liver disease. J. Gastroenterol. Hepatol. 2010, 25, 357-361. [CrossRef]

81. Ludwig, J.; Viggiano, T.R.; McGill, D.B.; Oh, B.J. Nonalcoholic steatohepatitis: Mayo Clinic experiences with a hitherto unnamed disease. Mayo Clin. Proc. 1980, 55, 434-438.

82. Sanyal, A.J. AGA technical review on nonalcoholic fatty liver disease. Gastroenterology 2002, 123, $1705-1725$. [CrossRef]

83. Neuschwander-Tetri, B.; Caldwell, S.H. Nonalcoholic steatohepatitis: Summary of an AASLD Single Topic Conference. Hepatology 2003, 37, 1202-1219. [CrossRef]

84. Joseph, A.E.; Saverymuttu, S.H.; Al-Sam, S.; Cook, M.G.; Maxwell, J.D. Comparison of liver histology with ultrasonography in assessing diffuse parenchymal liver disease. Clin. Radiol. 1991, 43, 26-31. [CrossRef]

85. Preiss, D.; Sattar, N. Non-alcoholic fatty liver disease: An overview of prevalence, diagnosis, pathogenesis and treatment considerations. Clin. Sci. 2008, 115, 141-150. [CrossRef]

86. Matteoni, C.; Younossi, Z.; Gramlich, T.; Boparai, N.; Liu, Y.; Mccullough, A. Nonalcoholic fatty liver disease: A spectrum of clinical and pathological severity. Gastroenterology 1999, 116, 1413-1419. [CrossRef]

87. Jung, C.H.; Kang, Y.M.; Jang, J.E.; Hwang, J.Y.; Kim, E.H.; Park, J.Y.; Kim, H.K.; Lee, W.J. Fatty liver index is a risk determinant of incident type 2 diabetes in a metabolically healthy population with obesity. Obesity 2016, 24, 1373-1379. [CrossRef]

88. Huh, J.H.; Ahn, S.V.; Koh, S.B.; Choi, E.; Kim, J.Y.; Sung, K.C.; Kim, E.J.; Park, J.B. A prospective study of fatty liver index and incident hypertension: The KoGES-ARIRANG study. PLoS ONE 2015, 10, e0143560. [CrossRef] [PubMed]

89. Lee, J.H.; Kim, D.; Kim, H.J.; Lee, C.H.; Yang, J.I.; Kim, W.; Kim, Y.J.; Yoon, J.H.; Cho, S.H.; Sung, M.W.; et al. Hepatic steatosis index: A simple screening tool reflecting nonalcoholic fatty liver disease. Dig. Liver Dis. 2010, 42, 503-508. [CrossRef]

90. Wang, J.; Xu, C.; Xun, Y.; Lu, Z.; Shi, J.; Yu, C.; Li, Y. ZJU index: A novel model for predicting nonalcoholic fatty liver disease in a Chinese population. Sci. Rep. 2015, 5, 1-10. [CrossRef]

91. Ruffillo, G.; Fassio, E.; Alvarez, E.; Landeira, G.; Longo, C.; Domínguez, N.; Gualano, G. Comparison of NAFLD fibrosis score and BARD score in predicting fibrosis in nonalcoholic fatty liver disease. J. Hepatol. 2011, 54, 160-163. [CrossRef] [PubMed]

92. Angulo, P.; Hui, J.M.; Marchesini, G.; Bugianesi, E.; George, J.; Farrell, G.C.; Enders, F.; Saksena, S.; Burt, A.D.; Bida, J.P.; et al. The NAFLD fibrosis score: A noninvasive system that identifies liver fibrosis in patients with NAFLD. Hepatology 2007, 45, 846-854. [CrossRef] [PubMed] 
93. Wai, C.T.; Greenson, J.K.; Fontana, R.J.; Kalbfleisch, J.D.; Marrero, J.A.; Conjeevaram, H.S.; Lok, A.S.F. Simple Noninvasive Index Can Predict Both Significant Fibrosis and Cirrhosis in Patients with Chronic Hepatitis C. Hepatology 2003, 38, 518-526. [CrossRef] [PubMed]

94. Sun, W.; Cui, H.; Li, N.; Wei, Y.; Lai, S.; Yang, Y.; Yin, X.; Chen, D.F. Comparison of FIB-4 index, NAFLD fibrosis score and BARD score for prediction of advanced fibrosis in adult patients with non-alcoholic fatty liver disease: A meta-analysis study. Hepatol. Res. 2016, 46, 862-870. [CrossRef]

95. Bedogni, G.; Bellentani, S.; Miglioli, L.; Masutti, F.; Passalacqua, M.; Castiglione, A.; Tiribelli, C. The fatty liver index: A simple and accurate predictor of hepatic steatosis in the general population. BMC Gastroenterol. 2006, 6, 1-7. [CrossRef]

96. Kahn, H.S. The "lipid accumulation product" performs better than the body mass index for recognizing cardiovascular risk: A population-based comparison. BMC Cardiovasc. Disord. 2005, 5, 1-10. [CrossRef]

97. Dai, H.; Wang, W.; Chen, R.; Chen, Z.; Lu, Y.; Yuan, H. Lipid accumulation product is a powerful tool to predict non-alcoholic fatty liver disease in Chinese adults. Nutr. Metab. 2017, 14, 1-9. [CrossRef]

98. Rotter, I.; Rył, A.; Szylińska, A.; Pawlukowska, W.; Lubkowska, A.; Laszczyńska, M. Lipid Accumulation Product (LAP) as an Index of Metabolic and Hormonal Disorders in Aging Men. Exp. Clin. Endocrinol. Diabetes 2017, 125, 176-182. [CrossRef]

99. Cuthbertson, D.J.; Weickert, M.O.; Lythgoe, D.; Sprung, V.S.; Dobson, R.; Shoajee-Moradie, F.; Umpleby, M.; Pfeiffer, A.F.H.; Thomas, E.L.; Bell, J.D.; et al. External validation of the fatty liver index and lipid accumulation product indices, using1H-magnetic resonance spectroscopy, to identify hepatic steatosis in healthy controls and obese, insulin-resistant individuals. Eur. J. Endocrinol. 2014, 171, 561-569. [CrossRef]

100. Harrison, S.A.; Oliver, D.; Arnold, H.L.; Gogia, S.; Neuschwander-Tetri, B.A. Development and validation of a simple NAFLD clinical scoring system for identifying patients without advanced disease. Gut 2008, 57, 1441-1447. [CrossRef]

101. Sterling, R.K.; Lissen, E.; Clumeck, N.; Sola, R.; Correa, M.C.; Montaner, J.; Sulkowski, M.S.; Torriani, F.J.; Dieterich, D.T.; Thomas, D.L.; et al. Development of a simple noninvasive index to predict significant fibrosis in patients with HIV/HCV coinfection. Hepatology 2006, 43, 1317-1325. [CrossRef]

102. Simopoulos, A.P. Human Requirement for N-3 Polyunsaturated Fatty Acids. Poult. Sci. 2000, 79, 961-970. [CrossRef]

103. Liguori, I.; Russo, G.; Curcio, F.; Bulli, G.; Aran, L.; Della-Morte, D.; Gargiulo, G.; Testa, G.; Cacciatore, F.; Bonaduce, D.; et al. Oxidative stress, aging, and diseases. Clin. Interv. Aging 2018, 13, 757-772. [CrossRef]

104. Patel, M.K.; Riley, M.A.; Hobbs, S.; Cortez-Cooper, M.; Robinson, V.J. Can $\alpha$-Lipoic Acid Mitigate Progression of Aging-Related Decline Caused by Oxidative Stress? South. Med. J. 2014, 107, 780-787. [CrossRef]

105. Sadowska-Bartosz, I.; Bartosz, G. Effect of antioxidants supplementation on aging and longevity. BioMed Res. Int. 2014, 2014, 1-17. [CrossRef]

106. Lorente-Cebrián, S.; Costa, A.G.V.; Navas-Carretero, S.; Zabala, M.; Martínez, J.A.; Moreno-Aliaga, M.J. Role of omega-3 fatty acids in obesity, metabolic syndrome, and cardiovascular diseases: A review of the evidence. J. Physiol. Biochem. 2013, 69, 633-651. [CrossRef]

107. Lorente-Cebrián, S.; Costa, A.G.V.; Navas-Carretero, S.; Zabala, M.; Laiglesia, L.M.; Martínez, J.A.; Moreno-Aliaga, M.J. An update on the role of omega-3 fatty acids on inflammatory and degenerative diseases. J. Physiol. Biochem. 2015, 71, 341-349. [CrossRef]

108. Martínez-Fernández, L.; Laiglesia, L.M.; Huerta, A.E.; Martínez, J.A.; Moreno-Aliaga, M.J. Omega-3 fatty acids and adipose tissue function in obesity and metabolic syndrome. Prostaglandins Other Lipid Mediat. 2015, 121, 24-41. [CrossRef]

109. Richard, D.; Kefi, K.; Barbe, U.; Bausero, P.; Visioli, F. Polyunsaturated fatty acids as antioxidants. Pharmacol. Res. 2008, 57, 451-455. [CrossRef]

110. Pettinelli, P.; del Pozo, T.; Araya, J.; Rodrigo, R.; Araya, A.V.; Smok, G.; Csendes, A.; Gutierrez, L.; Rojas, J.; Korn, O.; et al. Enhancement in liver SREBP-1c/PPAR- $\alpha$ ratio and steatosis in obese patients: Correlations with insulin resistance and n-3 long-chain polyunsaturated fatty acid depletion. Biochim. Biophys. Acta Mol. Basis Dis. 2009, 1792, 1080-1086. [CrossRef]

111. Spadaro, L.; Magliocco, O.; Spampinato, D.; Piro, S.; Oliveri, C.; Alagona, C.; Papa, G.; Rabuazzo, A.M.; Purrello, F. Effects of n-3 polyunsaturated fatty acids in subjects with nonalcoholic fatty liver disease. Dig. Liver Dis. 2008, 40, 194-199. [CrossRef] 
112. Zúñiga, J.; Cancino, M.; Medina, F.; Varela, P.; Vargas, R.; Tapia, G. N-3 PUFAS supplementation triggers PPAR- $\alpha$ activation and PPAR $-\alpha / \mathrm{NF}-\mathrm{KB}$ interaction: Anti-inflammatory implications in liver ischemia-reperfusion injury. PLoS ONE 2011, 6, e28502. [CrossRef]

113. Nenseter, M.S.; Drevon, C.A. Dietary polyunsaturates and peroxidation of low density lipoprotein. Curr. Opin. Lipidol. 1996, 7, 8-13. [CrossRef]

114. Liu, J.; Yeo, H.C.; Doniger, S.J.; Ames, B.N. Assay of aldehydes from lipid peroxidation: Gas chromatography-mass spectrometry compared to thiobarbituric acid. Anal. Biochem. 1997, 245, 161-166. [CrossRef]

115. Moreno-Aliaga, M.J.; Lorente-Cebrián, S.; Martínez, J.A. Regulation of adipokine secretion by n-3 fatty acids. Proc. Nutr. Soc. 2010, 69, 324-332. [CrossRef]

116. Hernandez-Rodas, M.C.; Valenzuela, R.; Videla, L.A. Relevant aspects of nutritional and dietary interventions in non-alcoholic fatty liver disease. Int. J. Mol. Sci. 2015, 16, 25168-25198. [CrossRef]

117. Softic, S.; Gupta, M.K.; Wang, G.-X.; Fujisaka, S.; O’Neill, B.T.; Rao, T.N.; Willoughby, J.; Harbison, C.; Fitzgerald, K.; Ilkayeva, O.; et al. Divergent effects of glucose and fructose on hepatic lipogenesis and insulin signaling. J. Clin. Investig. 2017, 127, 4059-4074. [CrossRef]

118. Yang, Z.H.; Miyahara, H.; Takeo, J.; Katayama, M. Diet high in fat and sucrose induces rapid onset of obesity-related metabolic syndrome partly through rapid response of genes involved in lipogenesis, insulin signalling and inflammation in mice. Diabetol. Metab. Syndr. 2012, 4, 1-10. [CrossRef]

119. Abdelmalek, M.F.; Suzuki, A.; Guy, C.; Unalp-Arida, A.; Colvin, R.; Johnson, R.J.; Diehl, A.M. Increased fructose consumption is associated with fibrosis severity in patients with nonalcoholic fatty liver disease. Hepatology 2010, 51, 1961-1971. [CrossRef] [PubMed]

120. Ter Horst, K.W.; Serlie, M.J. Fructose consumption, lipogenesis, and non-alcoholic fatty liver disease. Nutrients 2017, 9, 981. [CrossRef]

121. Crescenzo, R.; Bianco, F.; Falcone, I.; Coppola, P.; Liverini, G.; Iossa, S. Increased hepatic de novo lipogenesis and mitochondrial efficiency in a model of obesity induced by diets rich in fructose. Eur. J. Nutr. 2013, 52, 537-545. [CrossRef] [PubMed]

122. Aigner, E.; Theurl, I.; Haufe, H.; Seifert, M.; Hohla, F.; Scharinger, L.; Stickel, F.; Mourlane, F.; Weiss, G.; Datz, C. Copper Availability Contributes to Iron Perturbations in Human Nonalcoholic Fatty Liver Disease. Gastroenterology 2008, 135, 680-688. [CrossRef]

123. Aigner, E.; Strasser, M.; Haufe, H.; Sonnweber, T.; Hohla, F.; Stadlmayr, A.; Solioz, M.; Tilg, H.; Patsch, W.; Weiss, G.; et al. A Role for Low Hepatic Copper Concentrations in Nonalcoholic Fatty Liver Disease. Am. J. Gastroenterol. 2010, 105, 1978-1985. [CrossRef]

124. Aigner, E.; Weiss, G.; Datz, C. Dysregulation of iron and copper homeostasis in nonalcoholic fatty liver. World J. Hepatol. 2014, 7, 177. [CrossRef] [PubMed]

125. Schuster, S.; Cabrera, D.; Arrese, M.; Feldstein, A.E. Triggering and resolution of inflammation in NASH. Nat. Rev. Gastroenterol. Hepatol. 2018, 15, 349-364. [CrossRef] [PubMed]

126. Yan, J.H.; Guan, B.J.; Gao, H.Y.; Peng, X.E. Omega-3 polyunsaturated fatty acid supplementation and non-alcoholic fatty liver disease: A meta-analysis of randomized controlled trials. Medicine 2018, 97, e12271. [CrossRef] [PubMed]

127. Musa-Veloso, K.; Venditti, C.; Lee, H.Y.; Darch, M.; Floyd, S.; West, S.; Simon, R. Systematic review and meta-analysis of controlled intervention studies on the effectiveness of long-chain omega- 3 fatty acids in patients with nonalcoholic fatty liver disease. Nutr. Rev. 2018, 76, 581-602. [CrossRef]

128. Yu, L.; Yuan, M.; Wang, L. The effect of omega-3 unsaturated fatty acids on non-alcoholic fatty liver disease: A systematic review and meta-analysis of RCTs. Pak. J. Med. Sci. 2017, 33, 1022-1028. [CrossRef]

129. He, X.X.; Wu, X.L.; Chen, R.P.; Chen, C.; Liu, X.G.; Wu, B.J.; Huang, Z.M. Effectiveness of Omega-3 Polyunsaturated Fatty Acids in Non-Alcoholic Fatty Liver Disease: A Meta-Analysis of Randomized Controlled Trials. PLoS ONE 2016, 11, e0162368. [CrossRef]

130. Lu, W.; Li, S.; Li, J.; Wang, J.; Zhang, R.; Zhou, Y.; Yin, Q.; Zheng, Y.; Wang, F.; Xia, Y.; et al. Effects of Omega-3 Fatty Acid in Nonalcoholic Fatty Liver Disease: A Meta-Analysis. Gastroenterol. Res. Pract. 2016, 2016, 1459790. [CrossRef]

131. Scorletti, E.; Byrne, C.D. Omega-3 fatty acids and non-alcoholic fatty liver disease: Evidence of efficacy and mechanism of action. Mol. Asp. Med. 2018, 64, 135-146. [CrossRef] 
132. Kris-Etherton, P.; Taylor, D.S.; Yu-Poth, S.; Huth, P.; Moriarty, K.; Fishell, V.; Hargrove, R.L.; Zhao, G.; Etherton, T.D. Polyunsaturated fatty acids in the food chain in the United States. Am. J. Clin. Nutr. 2000, 71, 179S-188S. [CrossRef]

133. Gerster, H. Can adults adequately convert alpha-linolenic acid (18:3n-3) to eicosapentaenoic acid (20:5n-3) and docosahexaenoic acid (22:6n-3)? Int. J. Vitam. Nutr. Res. 1998, 68, 159-173. [PubMed]

134. Meyer, B.J.; Mann, N.J.; Lewis, J.L.; Milligan, G.C.; Sinclair, A.J.; Howe, P.R.C. Dietary intakes and food sources of omega-6 and omega-3 polyunsaturated fatty acids. Lipids 2003, 38, 391-398. [CrossRef]

135. Elmadfa, I.; Kornsteiner, M. Fats and fatty acid requirements for adults. Ann. Nutr. Metab. 2009, 55, 56-75. [CrossRef] [PubMed]

136. Kris-Etherton, P.M.; Harris, W.S.; Appel, L.J. Fish Consumption, Fish Oil, Omega-3 Fatty Acids, and Cardiovascular Disease. Circulation 2002, 106, 2747-2757. [CrossRef] [PubMed]

137. Simopoulos, A.P. Omega-3 fatty acids in health and disease and in growth and development. Am. J. Clin. Nutr. 1991, 54, 438-463. [CrossRef]

138. Sperling, R.I.; Austen, K.F. Effects of Omega-3 Polyunsaturated Fatty Acids on Human Leukocyte Function and Biochemistry. In Health Effects of Polyunsaturated Fatty Acids in Seafoods; Springer: Berlin/Heidelberg, Germany, 1987; pp. 227-238.

139. Seki, H.; Tani, Y.; Arita, M. Omega-3 PUFAS derived anti-inflammatory lipid mediator resolvin E1. Prostaglandins Other Lipid Mediat. 2009, 89, 126-130. [CrossRef] [PubMed]

140. Russo, G.L. Dietary n-6 and n-3 polyunsaturated fatty acids: From biochemistry to clinical implications in cardiovascular prevention. Biochem. Pharmacol. 2009, 77, 937-946. [CrossRef] [PubMed]

141. Jump, D.B.; Lytle, K.A.; Depner, C.M.; Tripathy, S. Omega-3 polyunsaturated fatty acids as a treatment strategy for nonalcoholic fatty liver disease. Pharmacol. Ther. 2018, 181, 108-125. [CrossRef]

142. George, E.S.; Forsyth, A.; Itsiopoulos, C.; Nicoll, A.J.; Ryan, M.; Sood, S.; Roberts, S.K.; Tierney, A.C. Practical dietary recommendations for the prevention andmanagement of nonalcoholic fatty liver disease in adults. Adv. Nutr. 2018, 9, 30-40. [CrossRef] [PubMed]

143. De Lorgeril, M.; Renaud, S.; Salen, P.; Monjaud, I.; Mamelle, N.; Martin, J.L.; Guidollet, J.; Touboul, P.; Delaye, J. Mediterranean alpha-linolenic acid-rich diet in secondary prevention of coronary heart disease. Lancet 1994, 343, 1454-1459. [CrossRef]

144. Ambring, A.; Johansson, M.; Axelsen, M.; Gan, L.M.; Strandvik, B.; Friberg, P. Mediterranean-inspired diet lowers the ratio of serum phospholipid n-6 to n-3 fatty acids, the number of leukocytes and platelets, and vascular endothelial growth factor in healthy subjects. Am. J. Clin. Nutr. 2006, 83, 575-581. [CrossRef]

145. Leclercq, I.A.; Molendi-Coste, O.; Legry, V. Why and how meet n-3 PUFA dietary recommendations? Gastroenterol. Res. Pract. 2011, 2011, 364040.

146. Simopoulos, A.P. An increase in the Omega-6/Omega-3 fatty acid ratio increases the risk for obesity. Nutrients 2016, 8, 128. [CrossRef] [PubMed]

147. Valenzuela, R.; Videla, L.A. The importance of the long-chain polyunsaturated fatty acid n-6/n-3 ratio in development of non-alcoholic fatty liver associated with obesity. Food Funct. 2011, 2, 644-648. [CrossRef] [PubMed]

148. Serhan, C.N. Systems approach to inflammation resolution: Identification of novel anti-inflammatory and pro-resolving mediators. J. Thromb. Haemost. 2009, 7, 44-48. [CrossRef]

149. Rius, B.; Duran-Güell, M.; Flores-Costa, R.; López-Vicario, C.; Lopategi, A.; Alcaraz-Quiles, J.; Casulleras, M.; José Lozano, J.; Titos, E.; Clària, J. The specialized proresolving lipid mediator maresin 1 protects hepatocytes from lipotoxic and hypoxia-induced endoplasmic reticulum stress. FASEB J. 2017, 31, 5384-5398. [CrossRef]

150. Martínez-Fernández, L.; González-Muniesa, P.; Laiglesia, L.M.; Sáinz, N.; Prieto-Hontoria, P.L.; Escoté, X.; Odriozola, L.; Corrales, F.J.; Arbones-Mainar, J.M.; Martínez, J.A.; et al. Maresin 1 improves insulin sensitivity and attenuates adipose tissue inflammation in ob/ob and diet-induced obese mice. FASEB J. 2017, 31, 2135-2145. [CrossRef]

151. Laiglesia, L.M.; Lorente-Cebrián, S.; Martínez-Fernández, L.; Sáinz, N.; Prieto-Hontoria, P.L.; Burrell, M.A.; Rodríguez-Ortigosa, C.M.; Martínez, J.A.; Moreno-Aliaga, M.J. Maresin 1 mitigates liver steatosis in ob/ob and diet-induced obese mice. Int. J. Obes. 2018, 42, 572-579. [CrossRef]

152. Rius, B.; Titos, E.; Morán-Salvador, E.; López-Vicario, C.; García-Alonso, V.; González-Périz, A.; Arroyo, V.; Clària, J. Resolvin D1 primes the resolution process initiated by calorie restriction in obesity-induced steatohepatitis. FASEB J. 2014, 28, 836-848. [CrossRef] 
153. Barden, A.; Mas, E.; Croft, K.D.; Phillips, M.; Mori, T.A. Short-term n-3 fatty acid supplementation but not aspirin increases plasma proresolving mediators of inflammation. J. Lipid Res. 2014, 55, 2401-2407. [CrossRef]

154. Jung, T.W.; Kim,H.C.; El-Aty, A.M.A.; Jeong, J.H. Maresin 1 attenuates NAFLD by suppression of endoplasmic reticulum stress via AMPK-SERCA2b pathway. J. Biol. Chem. 2018, 293, 3981-3988. [CrossRef]

155. González-Périz, A.; Planagumà, A.; Gronert, K.; Miquel, R.; López-Parra, M.; Titos, E.; Horrillo, R.; Ferré, N.; Deulofeu, R.; Arroyo, V.; et al. Docosahexaenoic acid (DHA) blunts liver injury by conversion to protective lipid mediators: Protectin D1 and 17S-hydroxy-DHA. FASEB J. 2006, 20, 2537-2539. [CrossRef]

156. González-Périz, A.; Horrillo, R.; Ferré, N.; Gronert, K.; Dong, B.; Morán-Salvador, E.; Titos, E.; Martínez-Clemente, M.; López-Parra, M.; Arroyo, V.; et al. Obesity-induced insulin resistance and hepatic steatosis are alleviated by $\omega-3$ fatty acids: A role for resolvins and protectins. FASEB J. 2009, 23, 1946-1957. [CrossRef]

157. Neuhofer, A.; Zeyda, M.; Mascher, D.; Itariu, B.K.; Murano, I.; Leitner, L.; Hochbrugger, E.E.; Fraisl, P.; Cinti, S.; Serhan, C.N.; et al. Impaired local production of proresolving lipid mediators in obesity and 17-HDHA as a potential treatment for obesity-associated inflammation. Diabetes 2013, 62, 1945-1956. [CrossRef]

158. Itariu, B.K.; Zeyda, M.; Hochbrugger, E.E.; Neuhofer, A.; Prager, G.; Schindler, K.; Bohdjalian, A.; Mascher, D.; Vangala, S.; Schranz, M.; et al. Long-chain n-3 PUFAs reduce adipose tissue and systemic inflammation in severely obese nondiabetic patients: A randomized controlled trial. Am. J. Clin. Nutr. 2012, 96, 1137-1149. [CrossRef]

159. Mori, T.A.; Dunstan, D.W.; Burke, V.; Croft, K.D.; Rivera, J.H.; Beilin, L.J.; Puddey, I.B. Effect of dietary fish and exercise training on urinary F2-isoprostane excretion in non-insulin-dependent diabetic patients. Metabolism 1999, 48, 1402-1408. [CrossRef]

160. Higdon, J.V.; Liu, J.; Du, S.-H.; Morrow, J.D.; Ames, B.N.; Wander, R.C. Supplementation of postmenopausal women with fish oil rich in eicosapentaenoic acid and docosahexaenoic acid is not associated with greater in vivo lipid peroxidation compared with oils rich in oleate and linoleate as assessed by plasma malondialdehyde and F2-isoprostanes. Am. J. Clin. Nutr. 2000, 72, 714-722.

161. Fayh, A.P.T.; Borges, K.; Cunha, G.S.; Krause, M.; Rocha, R.; de Bittencourt, P.I.H.; Moreira, J.C.F.; Friedman, R.; da Silva Rossato, J.; Fernandes, J.R.; et al. Effects of n-3 fatty acids and exercise on oxidative stress parameters in type 2 diabetic: A randomized clinical trial. J. Int. Soc. Sports Nutr. 2018, 15, 1-9. [CrossRef]

162. Mori, T.A.; Puddey, I.B.; Burke, V.; Croft, K.D.; Dunstan, D.W.; Rivera, J.H.; Beilin, L.J. Effect of $\omega 3$ fatty acids on oxidative stress in humans: GC-MS measurement of urinary $\mathrm{F}_{2}$-isoprostane excretion. Redox Rep. 2000, 5 , 45-46. [CrossRef]

163. Cazzola, R.; Russo-Volpe, S.; Miles, E.A.; Rees, D.; Banerjee, T.; Roynette, C.E.; Wells, S.J.; Goua, M.; Wahle, K.W.J.; Calder, P.C.; et al. Age- and dose-dependent effects of an eicosapentaenoic acid-rich oil on cardiovascular risk factors in healthy male subjects. Atherosclerosis 2007, 193, 159-167. [CrossRef] [PubMed]

164. Mas, E.; Woodman, R.J.; Burke, V.; Puddey, I.B.; Beilin, L.J.; Durand, T.; Mori, T.A. The omega-3 fatty acids EPA and DHA decrease plasma F2- isoprostanes: Results from two placebo-controlled interventions. Free Radic. Res. 2010, 44, 983-990. [CrossRef] [PubMed]

165. Schmidt, S.; Stahl, F.; Mutz, K.-O.; Scheper, T.; Hahn, A.; Schuchardt, J.P. Transcriptome-based identification of antioxidative gene expression after fish oil supplementation in normo- and dyslipidemic men. Nutr. Metab. 2012, 9, 45. [CrossRef]

166. Kiecolt-Glaser, J.K.; Epel, E.S.; Belury, M.A.; Andridge, R.; Lin, J.; Glaser, R.; Malarkey, W.B.; Hwang, B.S.; Blackburn, E. Omega-3 fatty acids, oxidative stress, and leukocyte telomere length: A randomized controlled trial. Brain Behav. Immun. 2013, 28, 16-24. [CrossRef]

167. Hajianfar, H.; Paknahad, Z.; Bahonar, A. The Effect of Omega-3 Supplements on Antioxidant Capacity in Patients with Type 2 Diabetes. Int. J. Prev. Med. 2013, 4, S234-S238. [PubMed]

168. Véricel, E.; Colas, R.; Calzada, C.; Lê, Q.H.; Feugier, N.; Cugnet, C.; Vidal, H.; Laville, M.; Moulin, P.; Lagarde, M. Moderate oral supplementation with docosahexaenoic acid improves platelet function and oxidative stress in type 2 diabetic patients. Thromb. Haemost. 2015, 114, 289-296. [PubMed]

169. Berge, R.K.; Ramsvik, M.S.; Bohov, P.; Svardal, A.; Nordrehaug, J.E.; Rostrup, E.; Bruheim, I.; Bjørndal, B. Krill oil reduces plasma triacylglycerol level and improves related lipoprotein particle concentration, fatty acid composition and redox status in healthy young adults-A pilot study. Lipids Health Dis. 2015, 14, 1-12. [CrossRef] [PubMed] 
170. Meydani, M.; Natiello, F.; Goldin, B.; Free, N.; Woods, M.; Schaefer, E.; Blumberg, J.B.; Gorbach, S.L. Effect of long-term fish oil supplementation on vitamin E status and lipid peroxidation in women. J. Nutr. 1991, 121, 484-491. [CrossRef]

171. Harats, D.; Dabach, Y.; Hollander, G.; Ben-Naim, M.; Schwartz, R.; Berry, E.M.; Stein, O.; Stein, Y. Fish oil ingestion in smokers and nonsmokers enhances peroxidation of plasma lipoproteins. Atherosclerosis 1991, 90, 127-139. [CrossRef]

172. Wander, R.C.; Du, S.-H. Oxidation of plasma proteins is not increased after supplementation with eicosapentaenoic and docosahexaenoic acids. Am. J. Clin. Nutr. 2000, 72, 731-737. [CrossRef] [PubMed]

173. Egert, S.; Somoza, V.; Kannenberg, F.; Fobker, M.; Krome, K.; Erbersdobler, H.F.; Wahrburg, U. Influence of three rapeseed oil-rich diets, fortified with alpha-linolenic acid, eicosapentaenoic acid or docosahexaenoic acid on the composition and oxidizability of low-density lipoproteins: Results of a controlled study in healthy volunteers. Eur. J. Clin. Nutr. 2007, 61, 314-325. [CrossRef]

174. Bloomer, R.J.; Larson, D.E.; Fisher-Wellman, K.H.; Galpin, A.J.; Schilling, B.K. Effect of eicosapentaenoic and docosahexaenoic acid on resting and exercise-induced inflammatory and oxidative stress biomarkers: A randomized, placebo controlled, cross-over study. Lipids Health Dis. 2009, 8, 1-12. [CrossRef] [PubMed]

175. Alves Luzia, L.; Mendes Aldrighi, J.; Teixeira Damasceno, N.R.; Rodrigues Sampaio, G.; Aparecida Manólio Soares, R.; Tande Silva, I.; De Queiroz Mello, A.P.; Ferreira Carioca, A.A.; Ferraz da Silva Torres, E.A. Fish Oil and Vitamin E Change Lipid Profiles and Anti-Ldl-Antibodies in Two Different Ethnic Groups of Women Transitioning through Menopause. Nutr. Hosp. 2015, 32, 165-174. [PubMed]

176. Nenseter, M.S.; Rustan, A.C.; Lund-Katz, S.; Søyland, E.; Maelandsmo, G.; Phillips, M.C.; Drevon, C.A. Effect of dietary supplementation with n-3 polyunsaturated fatty acids on physical properties and metabolism of low density lipoprotein in humans. Arterioscler. Thromb. 1992, 12, 369-379. [CrossRef] [PubMed]

177. Frankel, E.N.; Parks, E.J.; Xu, R.; Schneeman, B.O.; Davis, P.A.; German, J.B. Effect of n-3 fatty acid-rich fish oil supplementation on the oxidation of low density lipoproteins. Lipids 1994, 29, 233-236. [CrossRef]

178. Brude, I.R.; Drevon, C.A.; Hjermann, I.; Seljeflot, I.; Lund-Katz, S.; Saarem, K.; Sandstad, B.; Solvoll, K.; Halvorsen, B.; Arnesen, H.; et al. Peroxidation of LDL from combined-hyperlipidemic male smokers supplied with omega-3 fatty acids and antioxidants. Arterioscler. Thromb. Vasc. Biol. 1997, 17, 2576-2588. [CrossRef]

179. Wu, W.H.; Lu, S.C.; Wang, T.F.; Jou, H.J.; Wang, T.A. Effects of docosahexaenoic acid supplementation on blood lipids, estrogen metabolism, and in vivo oxidative stress in postmenopausal vegetarian women. Eur. J. Clin. Nutr. 2006, 60, 386-392. [CrossRef]

180. Hanwell, H.E.C.; Kay, C.D.; Lampe, J.W.; Holub, B.J.; Duncan, A.M. Acute Fish Oil and Soy Isoflavone Supplementation Increase Postprandial Serum (n-3) Polyunsaturated Fatty Acids and Isoflavones but Do Not Affect Triacylglycerols or Biomarkers of Oxidative Stress in Overweight and Obese Hypertriglyceridemic Men. J. Nutr. 2009, 139, 1128-1134. [CrossRef] [PubMed]

181. Petersson, H.; Risérus, U.; McMonagle, J.; Gulseth, H.L.; Tierney, A.C.; Morange, S.; Helal, O.; Shaw, D.I.; Ruano, J.A.; López-Miranda, J.; et al. Effects of dietary fat modification on oxidative stress and inflammatory markers in the LIPGENE study. Br. J. Nutr. 2010, 104, 1357-1362. [CrossRef]

182. Ulven, S.M.; Kirkhus, B.; Lamglait, A.; Basu, S.; Elind, E.; Haider, T.; Berge, K.; Vik, H.; Pedersen, J.I. Metabolic effects of krill oil are essentially similar to those of fish oil but at lower dose of EPA and DHA, in healthy volunteers. Lipids 2011, 46, 37-46. [CrossRef]

183. Ottestad, I.; Vogt, G.; Retterstøl, K.; Myhrstad, M.C.; Haugen, J.E.; Nilsson, A.; Ravn-Haren, G.; Nordvi, B.; Brønner, K.W.; Andersen, L.F.; et al. Oxidised fish oil does not influence established markers of oxidative stress in healthy human subjects: A randomised controlled trial. Br. J. Nutr. 2012, 108, 315-326. [CrossRef]

184. Egert, S.; Lindenmeier, M.; Harnack, K.; Krome, K.; Erbersdobler, H.F.; Wahrburg, U.; Somoza, V. Margarines fortified with $\alpha$-linolenic acid, eicosapentaenoic acid, or docosahexaenoic acid alter the fatty acid composition of erythrocytes but do not affect the antioxidant status of healthy adults. J. Nutr. 2012, 142, 1638-1644. [CrossRef] [PubMed]

185. Kirkhus, B.; Lamglait, A.; Eilertsen, K.E.; Falch, E.; Haider, T.; Vik, H.; Hoem, N.; Hagve, T.A.; Basu, S.; Olsen, E.; et al. Effects of similar intakes of marine n-3 fatty acids from enriched food products and fish oil on cardiovascular risk markers in healthy human subjects. Br. J. Nutr. 2012, 107, 1339-1349. [CrossRef]

186. Knasmüller, S.; Nersesyan, A.; Misík, M.; Gerner, C.; Mikulits, W.; Ehrlich, V.; Hoelzl, C.; Szakmary, A.; Wagner, K.H. Use of conventional and -omics based methods for health claims of dietary antioxidants: A critical overview. Br. J. Nutr. 2008, 99, ES3-ES52. [CrossRef] [PubMed] 
187. Dreissigacker, U.; Suchy, M.T.; Maassen, N.; Tsikas, D. Human plasma concentrations of malondialdehyde (MDA) and the F2-isoprostane 15(S)-8-iso-PGF2 $\alpha$ may be markedly compromised by hemolysis: Evidence by GC-MS/MS and potential analytical and biological ramifications. Clin. Biochem. 2010, 43, 159-167. [CrossRef] [PubMed]

188. Proudfoot, J.; Barden, A.; Mori, T.A.; Burke, V.; Croft, K.D.; Beilin, L.J.; Puddey, I.B. Measurement of urinary $\mathrm{F}_{2}$-Isoprostanes as markers of in vivo lipid peroxidation-A comparison of enzyme immunoassay with gas chromatography/mass spectrometry. Anal. Biochem. 1999, 272, 209-215. [CrossRef]

189. Smith, K.A.; Shepherd, J.; Wakil, A.; Kilpatrick, E.S. A comparison of methods for the measurement of 8-isoPGF(2alpha): A marker of oxidative stress. Ann. Clin. Biochem. 2011, 48, 147-154. [CrossRef] [PubMed]

190. Marrocco, I.; Altieri, F.; Peluso, I. Measurement and Clinical Significance of Biomarkers of Oxidative Stress in Humans. Oxid. Med. Cell. Longev. 2017, 2017, 6501046. [CrossRef]

191. Verhoye, E.; Langlois, M.R.; Asklepios Investigators. Circulating oxidized low-density lipoprotein: A biomarker of atherosclerosis and cardiovascular risk? Clin. Chem. Lab. Med. 2009, 47, 128-137. [CrossRef]

192. Kalousová, M.; Skrha, J.; Zima, T. Advanced glycation end-products and advanced oxidation protein products in patients with diabetes mellitus. Physiol. Res. 2002, 51, 597-604. [PubMed]

193. Du, S.L.; Zeng, X.Z.; Tian, J.W.; Ai, J.; Wan, J.; He, J.X. Advanced oxidation protein products in predicting acute kidney injury following cardiac surgery. Biomarkers 2015, 20, 206-211. [CrossRef]

194. Giustarini, D.; Dalle-Donne, I.; Tsikas, D.; Rossi, R. Oxidative stress and human diseases: Origin, link, measurement, mechanisms, and biomarkers. Crit. Rev. Clin. Lab. Sci. 2009, 46, 241-281. [CrossRef] [PubMed]

195. Tsikas, D.; Rothmann, S.; Schneider, J.Y.; Suchy, M.T.; Trettin, A.; Modun, D.; Stuke, N.; Maassen, N.; Frölich, J.C. Development, validation and biomedical applications of stable-isotope dilution GC-MS and GC-MS/MS techniques for circulating malondialdehyde (MDA) after pentafluorobenzyl bromide derivatization: MDA as a biomarker of oxidative stress and its relation to 15(S)-8-iso-prostaglandin F2 $\alpha$ and nitric oxide (NO). J. Chromatogr. B Anal. Technol. Biomed. Life Sci. 2016, 1019, 95-111.

196. Fenton, J.I.; Hord, N.G.; Ghosh, S.G. Long chain omega-3 fatty acid immunomodulation and the potencial for adverse healthoutcomes. Prostaglandins Leukot. Essent. Fat. Acids 2014, 89, 379-390. [CrossRef] [PubMed]

197. Hensley, K.; Robinson, K.A.; Gabbita, S.P.; Salsman, S.; Floyd, R.A. Reactive oxygen species, cell signaling, and cell injury. Free Radic. Biol. Med. 2000, 28, 1456-1462. [CrossRef]

198. Marseglia, L.; Manti, S.; D’Angelo, G.; Nicotera, A.; Parisi, E.; Di Rosa, G.; Gitto, E.; Arrigo, T. Oxidative Stress in Obesity: A Critical Component in Human Diseases. Int. J. Mol. Sci. 2014, 16, 378-400. [CrossRef]

199. Adkins, Y.; Kelley, D.S. Mechanisms underlying the cardioprotective effects of omega-3 polyunsaturated fatty acids. J. Nutr. Biochem. 2010, 21, 781-792. [CrossRef]

200. Capanni, M.; Calella, F.; Biagini, M.R.; Genise, S.; Raimondi, L.; Bedogni, G.; Svegliati-Baroni, G.; Sofi, F.; Milani, S.; Abbate, R.; et al. Prolonged n-3 polyunsaturated fatty acid supplementation ameliorates hepatic steatosis in patients with non-alcoholic fatty liver disease: A pilot study. Aliment. Pharmacol. Ther. 2006, 23, 1143-1151. [CrossRef]

201. Zhu, F.S.; Liu, S.; Chen, X.M.; Huang, Z.G.; Zhang, D.W. Effects of n-3 polyunsaturated fatty acids from seal oils on nonalcoholic fatty liver disease associated with hyperlipidemia. World J. Gastroenterol. 2008, 14, 6395-6400. [CrossRef]

202. Tanaka, N.; Sano, K.; Horiuchi, A.; Tanaka, E.; Kiyosawa, K.; Aoyama, T. Highly purified eicosapentaenoic acid treatment improves nonalcoholic steatohepatitis. J. Clin. Gastroenterol. 2008, 42, 413-418. [CrossRef]

203. Sofi, F.; Giangrandi, I.; Cesari, F.; Corsani, I.; Abbate, R.; Gensini, G.F.; Casini, A. Effects of a 1-year dietary intervention with n-3 polyunsaturated fatty acid-enriched olive oil on non-alcoholic fatty liver disease patients: A preliminary study. Int. J. Food Sci. Nutr. 2010, 61, 792-802. [CrossRef]

204. Scorletti, E.; Bhatia, L.; Mccormick, K.G.; Clough, G.F.; Nash, K.; Hodson, L.; Moyses, H.E.; Calder, P.C.; Byrne, C.D. Effects of purified eicosapentaenoic and docosahexaenoic acids in nonalcoholic fatty liver disease: Results from the WELCOME* study. Hepatology 2014, 60, 1211-1221. [CrossRef]

205. Sanyal, A.J.; Abdelmalek, M.F.; Suzuki, A.; Cummings, O.W.; Chojkier, M. No significant effects of ethyl-eicosapentanoic acid on histologic features of nonalcoholic steatohepatitis in a phase 2 triaL. Gastroenterology 2014, 147, 377-384. [CrossRef]

206. Li, Y.H.; Yang, L.H.; Sha, K.H.; Liu, T.G.; Zhang, L.G.; Liu, X.X. Efficacy of poly-unsaturated fatty acid therapy on patients with nonalcoholic steatohepatitis. World J. Gastroenterol. 2015, 21, 7008-7013. [CrossRef] 
207. Argo, C.K.; Patrie, J.T.; Lackner, C.; Henry, T.D.; de Lange, E.E.; Weltman, A.L.; Shah, N.L.; Al-Osaimi, A.M.; Pramoonjago, P.; Jayakumar, S.; et al. Effects of N-3 Fish Oil on Metabolic and Histological Parameters in NASH: A Double-Blind, Randomized, Placebo-Controlled Trial. J. Hepatol. 2015, 62, 190-197. [CrossRef]

208. Qin, Y.; Zhou, Y.; Chen, S.H.; Zhao, X.L.; Ran, L.; Zeng, X.L.; Wu, Y.; Chen, J.L.; Kang, C.; Shu, F.R.; et al. Fish oil supplements lower serum lipids and glucose in correlation with a reduction in plasma fibroblast growth factor 21 and prostaglandin E2 in nonalcoholic fatty liver disease associated with hyperlipidemia: A randomized clinical trial. PLoS ONE 2015, 10, e0133496. [CrossRef]

209. Dasarathy, S.; Dasarathy, J.; Khiyami, A.; Yerian, L.; Hawkins, C.; Sargent, R.; McCullough, A.J. Double-blind randomized placebo-controlled clinical trial of omega 3 fatty acids for the treatment of diabetic patients with nonalcoholic steatohepatitis. J. Clin. Gastroenterol. 2015, 49, 137-144. [CrossRef]

210. Nogueira, M.A.; Oliveira, C.P.; Ferreira Alves, V.A.; Stefano, J.T.; Rodrigues, L.S.; Torrinhas, R.S.; Cogliati, B.; Barbeiro, H.; Carrilho, F.J.; Waitzberg, D.L. Omega-3 polyunsaturated fatty acids in treating non-alcoholic steatohepatitis: A randomized, double-blind, placebo-controlled trial. Clin. Nutr. 2016, 35, 578-586. [CrossRef]

211. Tobin, D.; Brevik-Andersen, M.; Qin, Y.; Innes, J.K.; Calder, P.C. Evaluation of a High Concentrate Omega-3 for Correcting the Omega-3 Fatty Acid Nutritional Deficiency in Non-Alcoholic Fatty Liver Disease (CONDIN). Nutrients 2018, 10, 1126. [CrossRef]

212. Carpentier, Y.A.; Portois, L.; Malaisse, W.J. n-3 fatty acids and the metabolic syndrome. Am. J. Clin. Nutr. 2006, 83, 1499S-1504S. [CrossRef]

213. Satapathy, S.K.; Garg, S.; Chauhan, R.; Sakhuja, P.; Malhotra, V.; Sharma, B.C.; Sarin, S.K. Beneficial effects of tumor necrosis factor- $\alpha$ inhibition by pentoxifylline on clinical, biochemical, and metabolic parameters of patients with nonalcoholic steatohepatitis. Am. J. Gastroenterol. 2004, 99, 1946-1952. [CrossRef] [PubMed]

214. Lee, D.H.; Jacobs, D.R. Association between serum gamma-glutamyltransferase and C-reactive protein. Atherosclerosis 2005, 178, 327-330. [CrossRef] [PubMed]

215. Manco, M.; Marcellini, M.; Giannone, G.; Nobili, V. Correlation of serum TNF- $\alpha$ levels and histologic liver injury scores in pediatric nonalcoholic fatty liver disease. Am. J. Clin. Pathol. 2007, 127, 954-960. [CrossRef]

216. Serviddio, G.; Bellanti, F.; Vendemiale, G. Free radical biology for medicine: Learning from nonalcoholic fatty liver disease. Free Radic. Biol. Med. 2013, 65, 952-968. [CrossRef]

217. Svegliati-Baroni, G.; Candelaresi, C.; Saccomanno, S.; Ferretti, G.; Bachetti, T.; Marzioni, M.; De Minicis, S.; Nobili, L.; Salzano, R.; Omenetti, A.; et al. A model of insulin resistance and nonalcoholic steatohepatitis in rats: Role of peroxisome proliferator-activated receptor- $\alpha$ and $n-3$ polyunsaturated fatty acid treatment on liver injury. Am. J. Pathol. 2006, 169, 846-860. [CrossRef]

218. Maratos-Flier, E. Fatty liver and FGF21 physiology. Exp. Cell Res. 2017, 360, 2-5. [CrossRef]

219. Scorletti, E.; Byrne, C.D. Omega-3 Fatty Acids, Hepatic Lipid Metabolism, and Nonalcoholic Fatty Liver Disease. Annu. Rev. Nutr. 2013, 33, 231-248. [CrossRef]

220. Nobili, V.; Carpino, G.; Alisi, A.; De Vito, R.; Franchitto, A.; Alpini, G.; Onori, P.; Gaudio, E. Role of docosahexaenoic acid treatment in improving liver histology in pediatric nonalcoholic fatty liver disease. PLoS ONE 2014, 9, e88005. [CrossRef]

221. Pacifico, L.; Bonci, E.; Di Martino, M.; Versacci, P.; Andreoli, G.; Silvestri, L.M.; Chiesa, C. A double-blind, placebo-controlled randomized trial to evaluate the efficacy of docosahexaenoic acid supplementation on hepatic fat and associated cardiovascular risk factors in overweight children with nonalcoholic fatty liver disease. Nutr. Metab. Cardiovasc. Dis. 2015, 25, 734-741. [CrossRef]

222. Wang, J.Z.; Cao, H.X.; Chen, J.N.; Pan, Q. PNPLA3 rs738409 underlies treatment response in nonalcoholic fatty liver disease. World J. Clin. Cases 2018, 6, 167-175. [CrossRef] [PubMed]

223. Okada, L.S.D.R.R.; Oliveira, C.P.; Stefano, J.T.; Nogueira, M.A.; Silva, I.D.C.G.D.; Cordeiro, F.B.; Alves, V.A.F.; Torrinhas, R.S.; Carrilho, F.J.; Puri, P.; et al. Omega-3 PUFA modulate lipogenesis, ER stress, and mitochondrial dysfunction markers in NASH-Proteomic and lipidomic insight. Clin. Nutr. 2018, 37, 1474-1484. [CrossRef] [PubMed]

(C) 2019 by the authors. Licensee MDPI, Basel, Switzerland. This article is an open access article distributed under the terms and conditions of the Creative Commons Attribution (CC BY) license (http://creativecommons.org/licenses/by/4.0/). 